\title{
On Approximate Controllability of Second-Order Neutral Partial Stochastic Functional Integrodifferential Inclusions with Infinite Delay and Impulsive Effects
}

\author{
Zuomao Yan \\ Department of Mathematics, Hexi University, Zhangye, Gansu 734000, China \\ Correspondence should be addressed to Zuomao Yan; yanzuomao@163.com
}

Received 20 January 2015; Revised 16 April 2015; Accepted 19 April 2015

Academic Editor: Mark A. McKibben

Copyright (C) 2015 Zuomao Yan. This is an open access article distributed under the Creative Commons Attribution License, which permits unrestricted use, distribution, and reproduction in any medium, provided the original work is properly cited.

\begin{abstract}
We discuss the approximate controllability of second-order impulsive neutral partial stochastic functional integrodifferential inclusions with infinite delay under the assumptions that the corresponding linear system is approximately controllable. Using the fixed point strategy, stochastic analysis, and properties of the cosine family of bounded linear operators combined with approximation techniques, a new set of sufficient conditions for approximate controllability of the second-order impulsive partial stochastic integrodifferential systems are formulated and proved. The results in this paper are generalization and continuation of the recent results on this issue. An example is provided to show the application of our result.
\end{abstract}

\section{Introduction}

Impulsive effects exist widely in many evolution processes in which states are changed abruptly at certain moments of time, involving fields such as physics, chemical technology, population dynamics, biotechnology, and economics; see [1-4] and the references therein. However, in addition to impulsive effects, stochastic effects likewise exist in real systems. A lot of dynamical systems have variable structures subject to stochastic abrupt changes, which may result from abrupt phenomena such as stochastic failures and repairs of the components, changes in the interconnections of subsystems, sudden environment changes, and other areas of science. Therefore, it is necessary and important to consider the impulsive stochastic dynamical systems. Particularly, the authors in [5-7] studied the existence of mild solutions for a class of abstract impulsive neutral stochastic functional differential and integrodifferential equations with infinite delay in Hilbert spaces.

The concept of controllability leads to some very important conclusions regarding the behavior of linear and nonlinear dynamical systems. In the case of infinite-dimensional systems, two basic concepts of controllability can be distinguished. There are exact and approximate controllability.
However, the concept of exact controllability is usually too strong [8]. Therefore, approximate controllability problems for deterministic and stochastic dynamical systems in infinite dimensional spaces are well developed using different kind of approaches (see $[9,10])$. Stochastic control theory is a stochastic generalization of classic control theory. So significant progress has been made in the approximate controllability of linear and nonlinear stochastic systems in Banach spaces (see, e.g., [9-12]). Several papers [13-16] have appeared on the approximate controllability of nonlinear impulsive stochastic differential systems in Hilbert spaces.

In many cases, it is advantageous to treat the secondorder stochastic differential equations directly rather than to convert them to first-order systems. The second-order stochastic differential equations are the right model in continuous time to account for integrated processes that can be made stationary. Recently, based on the fixed point theory, the existence and approximate controllability of mild solutions for various second-order stochastic partial differential equations and impulsive stochastic partial differential equations have been extensively studied. For example, Ren and Sun [17], Cui and Yan [18], and Mahmudov and McKibben [19] proved the approximate controllability of second-order 
neutral stochastic evolution differential equations. Muthukumar and Balasubramaniam [20] established sufficient conditions for the approximate controllability of a class of secondorder nonlinear stochastic functional differential equations of McKean-Vlasov type. Balasubramaniam and Muthukumar in [21] discussed the approximate controllability of secondorder neutral stochastic distributed implicit functional differential equations with infinite delay. Sakthivel et al. in [22] studied the approximate controllability of second-order impulsive stochastic differential equations. On the other hand, many systems arising from realistic models can be described as partial stochastic differential or integrodifferential inclusions (see [23-27] and references therein), so it is natural to extend the concept controllability of mild solution for second-order impulsive stochastic evolution equations to second-order impulsive systems represented by stochastic partial differential or integrodifferential inclusions. In this paper, we consider the approximate controllability of the following second-order impulsive neutral partial stochastic functional integrodifferential inclusions with infinite delay in Hilbert spaces of the form

$$
\begin{aligned}
& d\left[x^{\prime}(t)-g\left(t, x_{t}, x^{\prime}(t)\right)\right] \\
& \quad \in[A x(t)+B u(t)] d t \\
& \quad+F\left(t, x_{t}, x^{\prime}(t), \int_{0}^{t} h\left(t, s, x_{s}, x^{\prime}(s)\right) d s\right) d w(t), \\
& \quad t \in J, t \neq t_{k}, \\
& \Delta x\left(t_{k}\right)=I_{k}\left(x\left(t_{k}\right), x^{\prime}\left(t_{k}\right)\right), \quad k=1, \ldots, m, \\
& \Delta x^{\prime}\left(t_{k}\right)=J_{k}\left(x\left(t_{k}\right), x^{\prime}\left(t_{k}\right)\right), \quad k=1, \ldots, m, \\
& x_{0}=\varphi \in \mathscr{B}, \\
& x^{\prime}(0)=\phi,
\end{aligned}
$$

where the state $x(\cdot)$ takes values in a separable real Hilbert space $H$ with inner product $\langle\cdot, \cdot\rangle_{H}$ and norm $\|\cdot\|_{H}$. The operator $A: D(A) \rightarrow H$ is the infinitesimal generator of a strongly continuous cosine family on $H$. The control function $u \in L^{p}(J, U)$, a Hilbert space of admissible control functions, $p \geq 2$ is an integer, and $B$ is a bounded linear operator from a Banach space $U$ to $H$. Let $K$ be another separable Hilbert space with inner product $\langle\cdot, \cdot\rangle_{K}$ and norm $\|\cdot\|_{K}$. Suppose that $\{w(t): t \geq 0\}$ is a given $K$-valued Wiener process with a covariance operator $Q>0$ defined on a complete probability space $(\Omega, \mathscr{F}, P)$ equipped with a normal filtration $\left\{\mathscr{F}_{t}\right\}_{t>0}$, which is generated by the Wiener process $w$. The time history $x_{t}:(-\infty, 0] \rightarrow H$ given by $x_{t}(\theta)=x(t+\theta)$ belongs to some abstract phase space $\mathscr{B}$ defined axiomatically; $F, g, h$, $I_{k}, J_{k}(k=1, \ldots, m)$ are given functions to be specified later. Moreover, let $0<t_{1}<\cdots<t_{m}<b$ be prefixed points and the symbol $\Delta x\left(t_{k}\right)=x\left(t_{k}^{+}\right)-x\left(t_{k}^{-}\right)$, where $x\left(t_{k}^{-}\right)$and $x\left(t_{k}^{+}\right)$ represent the right and left limits of $x(t)$ at $t=t_{k}$, respectively. The initial data $\{\varphi(t):-\infty<t \leq 0\}$ is an $\mathscr{F}_{0}$-adapted, $\mathscr{B}$ valued random variable independent of the Wiener process $w$ with finite second moment.
To the best of the author's knowledge, there are no results about the existence and approximate controllability of mild solutions for second-order impulsive second-order neutral partial stochastic functional integrodifferential inclusions with infinite delay, which is expressed in the form of (1). In order to fill this gap, this paper studies this interesting problem. We derive the sufficient conditions for the approximate controllability of system (1) by using the fixed point theorem for multivalued mapping due to Dhage [28] with stochastic analysis and properties of the cosine family of bounded linear operators combined with approximation techniques. The obtained result can be seen as a contribution to this emerging field. Moreover, the operators $I_{k}, J_{k}(k=1, \ldots, m)$ are continuous but without imposing completely continuous and Lipschitz condition. The results shown are also new for deterministic second-order systems with impulsive effects.

The rest of this paper is organized as follows. In Section 2, we introduce some notations and necessary preliminaries. Section 3 verifies the existence of solutions for impulsive stochastic control system (1). In Section 4 we establish the approximate controllability of impulsive stochastic control system (1). Finally in Section 5, an example is given to illustrate our results.

\section{Preliminaries}

Let $(\Omega, \mathscr{F}, P)$ be a complete probability space equipped with a normal filtration $\mathscr{F}_{t}, t \in[0, b]$. Let $H$ and $K$ be the separable Hilbert spaces and let $w$ be a $Q$-Weiner process on $\left(\Omega, \mathscr{F}_{b}, P\right)$ with the covariance operator $Q$ such that $\operatorname{tr} Q<$ $\infty$. We assume that there exists a complete orthonormal system $\left\{e_{n}\right\}_{n=1}^{\infty}$ in $K$, a bounded sequence of nonnegative real numbers $\left\{\lambda_{n}\right\}_{n=1}^{\infty}$ such that $Q e_{i}=\lambda_{i} e_{i}$, and a sequence $\beta_{i}$ of independent Brownian motions such that

$$
\langle w(t), e\rangle=\sum_{n=1}^{\infty} \sqrt{\lambda_{n}}\left\langle e_{n}, e\right\rangle \beta_{n}(t), \quad e \in K, t \in J,
$$

and $\mathscr{F}_{t}=\mathscr{F}_{t}^{w}$, where $\mathscr{F}_{t}^{w}$ is the $\sigma$-algebra generated by $\{w(s)$ : $0 \leq s \leq t\}$. Let $L_{0}^{2}=L^{2}\left(Q^{1 / 2} K ; H\right)$ be the space of all HilbertSchmidt operators from $Q^{1 / 2} K$ to $H$ with the inner product $\langle\psi, \theta\rangle_{L_{0}^{2}}=\operatorname{Tr}\left(\psi Q \theta^{*}\right)$. Let $L^{p}\left(\mathscr{F}_{b}, H\right)$ be the Banach space of all $\mathscr{F}_{b}$-measurable $p$ th power integrable random variables with values in the Hilbert space $H$. Let $C\left([0, b] ; L^{p}(\mathscr{F}, H)\right)$ be the Banach space of continuous maps from $[0, b]$ into $L^{p}(\mathscr{F}, H)$ satisfying the condition $\sup _{t \in J} E\|x(t)\|_{H}^{p}<\infty$.

We use the notations that $\mathscr{P}(H)$ is the family of all subsets of $H$. Let us introduce the following notations:

$$
\begin{aligned}
& \mathscr{P}_{\mathrm{cl}}(H)=\{x \in \mathscr{P}(H): x \text { is closed }\}, \\
& \mathscr{P}_{\mathrm{bd}}(H)=\{x \in \mathscr{P}(H): x \text { is bounded }\}, \\
& \mathscr{P}_{\mathrm{cv}}(H)=\{x \in \mathscr{P}(H): x \text { is convex }\}, \\
& \mathscr{P}_{\mathrm{cp}}(H)=\{x \in \mathscr{P}(H): x \text { is compact }\} .
\end{aligned}
$$


Consider $H_{d}: \mathscr{P}(H) \times \mathscr{P}(H) \rightarrow \mathbb{R}^{+} \cup\{\infty\}$ given by

$$
H_{d}(\widetilde{A}, \widetilde{B})=\max \left\{\sup _{\widetilde{a} \in \widetilde{A}} d(\widetilde{a}, \widetilde{B}), \sup _{\widetilde{b} \in \widetilde{B}} d(\widetilde{A}, \widetilde{a})\right\},
$$

where $d(\widetilde{A}, \widetilde{b})=\inf _{\widetilde{a} \in \widetilde{A}} d(\widetilde{a}, \widetilde{b})$ and $d(\widetilde{a}, \widetilde{B})=\inf _{\widetilde{b} \in \widetilde{B}} d(\widetilde{a}, \widetilde{b})$. Then $\left(\mathscr{P}_{\text {bd,cl }}(H), H_{d}\right)$ is a metric space and $\left(\mathscr{P}_{\mathrm{cl}}(H), H_{d}\right)$ is a generalized metric space.

In what follows, we briefly introduce some facts on multivalued analysis. For more details, one can see [29, 30].

A multivalued map $\Phi: H \rightarrow \mathscr{P}(H)$ is convex (closed) valued if $G(H)$ is convex (closed) for all $x \in H$. $\Phi$ is bounded on bounded sets if $\Phi(D)=\bigcup_{x \in D} \Phi(x)$ is bounded in $H$ for any bounded set $D$ of $H$; that is, $\sup _{x \in D}\left\{\sup \left\{\|y\|_{H}: y \in\right.\right.$ $\Phi(x)\}\}<\infty$.

$\Phi$ is called upper semicontinuous (u.s.c., in short) on $H$, if, for any $x \in H$, the set $\Phi(x)$ is a nonempty, closed subset of $H$ and if, for each open set $B$ of $H$ containing $\Phi(x)$, there exists an open neighborhood $N$ of $x$ such that $\Phi(N) \subseteq B$.

$\Phi$ is said to be completely continuous if $\Phi(D)$ is relatively compact for every bounded subset $D$ of $H$. If the multivalued map $\Phi$ is completely continuous with nonempty compact values, then $\Phi$ is u.s.c. if and only if $\Phi$ has a closed graph; that is, $x_{n} \rightarrow x_{*}, y_{n} \rightarrow y_{*}$, and $y_{n} \in \Phi\left(x_{n}\right)$ imply $y_{*} \in \Phi\left(x_{*}\right)$.

$\Phi$ is said to be completely continuous if $\Phi(D)$ is relatively compact, for every bounded subset $D \subseteq H$.

A multivalued map $\Phi: J \rightarrow \mathscr{P}_{\mathrm{cl}}$ is said to be measurable if, for each $x \in H$, the function $Y: J \rightarrow \mathbb{R}^{+}$defined by $Y(t)=d(x, \Phi(t))=\inf \{d(x, z): z \in \Phi(t)\}$ is measurable.

In this paper, $A$ is the infinitesimal generator of a strongly continuous cosine function of bounded linear operators $(C(t))_{t \in \mathbb{R}}$ on $H$. The corresponding strongly continuous sine family $\{S(t): t \in \mathbb{R}\} \subset L(H)$ is defined by $S(t) x=\int_{0}^{t} C(s) x d s$, $t \in \mathbb{R}, x \in H$. The generator $A: H \rightarrow H$ of $\{C(t): t \in \mathbb{R}\}$ is given by $A x=\left.\left(d^{2} / d t^{2}\right) C(t) x\right|_{t=0}$ for all $x \in D(A)=$ $\left\{x \in H: C(\cdot) x \in C^{2}(\mathbb{R}, H)\right\}$. It is well known that the infinitesimal generator $A$ is a closed densely defined operator on $H$. As usual we denote by $[D(A)]$ the domain of operator $A$ endowed with the graph norm $\|x\|_{A}=\|x\|_{H}+\|A x\|_{H}, \quad x \in$ $D(A)$. Moreover, the notation $\mathbb{E}$ stands for the space formed by the vectors $x \in H$ for which $C(\cdot) x$ is of class $C^{1}$ on $\mathbb{R}$. It was proved by Kisyński [31] that $\mathbb{E}$ endowed with the norm $\|x\|_{\mathbb{E}}=\|x\|_{H}+\sup _{0 \leq t \leq 1}\|A S(t) x\|_{H}, x \in \mathbb{E}$, is a Banach space. Such cosine and corresponding sine families and their generators satisfy that the following properties.

Lemma 1 (see [32]). Suppose that $A$ is the infinitesimal generator of a cosine family of operators $\{C(t): t \in \mathbb{R}\}$. Then, the following hold:

(a) There exist $\widetilde{M}_{1} \geq 1$ and $\alpha \geq 0$ such that $\|C(t)\|_{H} \leq$ $\widetilde{M}_{1} e^{\alpha t}$ and hence $\|S(t)\|_{H} \leq \widetilde{M}_{1} e^{\alpha t}$.

(b) Consider $A \int_{s}^{r} S(u) x d u=[C(r)-C(u)] x$ for all $0 \leq$ $s \leq r<\infty$.

(c) There exists $\widetilde{M}_{2} \geq 1$ such that $\|S(s)-S(r)\|_{H} \leq$ $\widetilde{M}_{2}\left|\int_{r}^{s} e^{\alpha|\theta|} d \theta\right|$ for all $0 \leq r \leq s<\infty$.
The existence of solutions of the second-order linear abstract Cauchy problem

$$
\begin{aligned}
x^{\prime \prime}(t) & =A x(t)+F(t), \quad t \in J, \\
x(0) & =z_{0}, \\
x^{\prime}(0) & =z_{1},
\end{aligned}
$$

where $h: J \rightarrow H$ is an integrable function, has been discussed in [33]. Similarly, the existence of solutions for semilinear second-order abstract Cauchy problem has been treated in [32]. We only mention here that the function $x(\cdot)$ given by

$$
x(t)=C(t) z_{0}+S(t) z_{1}+\int_{0}^{t} S(t-s) F(s) d s, \quad t \in J,
$$

is called a mild solution of (5) and if $z_{1} \in \mathbb{E}$, the function $x(\cdot)$ is continuously differentiable and

$$
x^{\prime}(t)=A S(t) z_{0}+C(t) z_{1}+\int_{0}^{t} C(t-s) F(s) d s,
$$

$t \in J$.

A function $x:[\mu, \tau] \rightarrow H$ is said to be normalized piecewise continuous function on $[\mu, \tau]$ if $x$ is piecewise continuous and left continuous on $(\mu, \tau]$. We denote by $\mathscr{P} \mathscr{C}([\mu, \tau], H)$ the space formed by the normalized piecewise continuous, $\mathscr{F}_{t}$-adapted measurable processes from $[\mu, \tau]$ into $H$. In particular, we introduce the space $\mathscr{P} \mathscr{C}$ formed by all $\mathscr{F}_{t}$-adapted measurable, $H$-valued stochastic processes $\{x(t): t \in[0, b]\}$ such that $x$ is continuous at $t \neq t_{k}, x\left(t_{k}\right)=$ $x\left(t_{k}^{-}\right)$and $x\left(t_{k}^{+}\right)$exists for $k=1,2, \ldots, m$. Similarly, $\mathscr{P}_{\mathscr{C}}^{1}$ formed by all $\mathscr{F}_{t}$-adapted measurable, $H$-valued stochastic processes $\left\{x^{\prime}(t): t \in[0, b]\right\}$ such that $x^{\prime}$ is continuous at $t \neq t_{k}, x^{\prime}\left(t_{k}\right)=x^{\prime}\left(t_{k}^{-}\right)$, and $x^{\prime}\left(t_{k}^{+}\right)$exists for $k=1,2, \ldots, m$. In this paper, we always assume that $\mathscr{P} \mathscr{C}$ is endowed with the norm $\|x\|_{\mathscr{P} \mathscr{C}}=\left(\sup _{0 \leq t \leq b} E\|x(t)\|_{H}^{p}\right)^{1 / p}$. Then $\left(\mathscr{P} \mathscr{C},\|\cdot\|_{\mathscr{P} \mathscr{C}}\right)$ is a Banach space. Next, for $x \in \mathscr{P} \mathscr{C}^{1}$, we represent by $x^{\prime}(t)$ the right derivative at $t \in(0, b]$ and by $x^{\prime}(0)$ the right derivative at zero. It is easy to see that $\mathscr{P} \mathscr{C}^{1}$ is provided with the norm $\|x\|_{\mathscr{P} \mathscr{C}^{1}}=\|x\|_{\mathscr{P} \mathscr{C}}+\left\|x^{\prime}\right\|_{\mathscr{P} \mathscr{C}}$ being a Banach space.

In this paper, we assume that the phase space $\left(\mathscr{B},\|\cdot\|_{\mathscr{B}}\right)$ is a seminormed linear space of $\mathscr{F}_{0}$-measurable functions mapping $(-\infty, 0]$ into $H$ and satisfying the following fundamental axioms due to Hale and Kato (see, e.g., [34]).

(A) If $x:(-\infty, \sigma+b] \rightarrow H, b>0$, such that $\left.x\right|_{[\sigma, \sigma+b]} \in$ $\mathscr{P} \mathscr{C}^{1}([\sigma, \sigma+b], H)$ and $x_{\sigma} \in \mathscr{B}$, then for every $t \in$ $[\sigma, \sigma+b]$ the following conditions hold:

(i) $x_{t}$ is in $\mathscr{B}$;

(ii) $\|x(t)\|_{H} \leq \widetilde{H}\left\|x_{t}\right\|_{\mathscr{B}}$;

(iii) $\left\|x_{t}\right\|_{\mathscr{B}} \leq K(t-\sigma) \sup \left\{\|x(s)\|_{H}: \sigma \leq s \leq\right.$ $t\}+M(t-\sigma)\left\|x_{\sigma}\right\|_{\mathscr{B}}$, where $\widetilde{H} \geq 0$ is a constant; $K, M:[0, \infty) \rightarrow[1, \infty), K$ is continuous and $M$ is locally bounded, and $\widetilde{H}, K$, and $M$ are independent of $x(\cdot)$. 
(B) For the function $x(\cdot)$ in (A), the function $t \rightarrow x_{t}$ is continuous from $[\sigma, \sigma+b]$ into $\mathscr{B}$.

(C) The space $\mathscr{B}$ is complete.

Example 2. The phase space $\mathscr{P} \mathscr{C}_{r} \times L^{p}(\tilde{h}, H)$. Let $r \geq 0$, $1 \leq p<+\infty$ and let $\tilde{h}:(-\infty,-r] \rightarrow \mathbb{R}$ be a nonnegative measurable function which satisfies conditions (h-5) and (h6 ) in the terminology of Hino et al. [35]. Briefly, this means that $\tilde{h}$ is locally integrable and there is a nonnegative, locally bounded function $\gamma$ on $(-\infty, 0]$ such that $\widetilde{h}(\xi+\tau) \leq \gamma(\xi) \widetilde{h}(\tau)$ for all $\xi \leq 0$ and $\xi \in(-\infty,-r) \backslash N_{\xi}$, where $N_{\xi} \subseteq(-\infty,-r)$ is a set whose Lebesgue measure is zero. We denote by $\mathscr{P} \mathscr{C}_{r} \times$ $L^{p}(\widetilde{h}, H)$ the set consisting of all classes of functions $\varphi$ : $(-\infty, 0] \rightarrow H$ such that $\varphi_{\left.\right|_{[-r, 0]}} \in \mathscr{P} \mathscr{C}([-r, 0], H), \varphi(\cdot)$ is Lebesgue measurable on $(-\infty,-r)$, and $\widetilde{h}\|\varphi\|^{p}$ is Lebesgue integrable on $(-\infty,-r)$. The seminorm is given by

$$
\|\varphi\|_{\mathscr{B}}=\sup _{-r \leq \tau \leq 0}\|\varphi(\tau)\|+\left(\int_{-\infty}^{-r} \tilde{h}(\tau)\|\varphi\|^{p} d \tau\right)^{1 / p} .
$$

The space $\mathscr{B}=\mathscr{P} \mathscr{C}_{r} \times L^{p}(\widetilde{h}, H)$ satisfies axioms $(\mathrm{A})-(\mathrm{C})$. Moreover, when $r=0$ and $p=2$, we can take $\widetilde{H}=1, M(t)=$ $\gamma(-t)^{1 / 2}$, and $K(t)=1+\left(\int_{-t}^{0} \tilde{h}(\tau) d \tau\right)^{1 / 2}$, for $t \geq 0$ (see [35, Theorem 1.3.8] for details).

Remark 3 (see [4]). In retarded functional differential equations without impulses, the axioms of the abstract phase space $\mathscr{B}$ include the continuity of the function $t \rightarrow x_{t}$. Due to the impulsive effect, this property is not satisfied in impulsive delay systems and, for this reason, has been eliminated in our abstract description of $\mathscr{B}$.

Remark 4. In the rest of this paper $M_{b}$ and $K_{b}$ are the constants defined by $M_{b}=\sup _{t \in J} M(t)$ and $K_{b}=\sup _{t \in J} K(t)$.

For $x \in \mathscr{P} \mathscr{C}$, we denote by $\widehat{x}_{k}, k=0,1, \ldots, m$, the unique continuous function $\widehat{x}_{k} \in C\left(\left[t_{k}, t_{k+1}\right] ; H\right)$ such that

$$
\widehat{x}_{k}(t):= \begin{cases}x(t) & \text { for } t \in\left(t_{k}, t_{k+1}\right], \\ x\left(t_{k}^{+}\right) & \text {for } t=t_{k} .\end{cases}
$$

Moreover, for $B \subseteq \mathscr{P} \mathscr{C}$ we denote by $\widehat{B}_{k}, k=0,1, \ldots, m$, the set $\widehat{B}_{k}=\left\{\widehat{x}_{k}: x \in B\right\}$. The notation $B_{r}(x, H)$ stands for the closed ball with center at $x$ and radius $r>0$ in $H$.

Lemma 5. $A$ set $B \subseteq \mathscr{P} \mathscr{C}$ is relatively compact in $\mathscr{P} \mathscr{C}$ if and only if the set $\widehat{B}_{k}$ is relatively compact in $C\left(\left[t_{k}, t_{k+1}\right] ; L_{2}(\Omega, H)\right)$, for every $k=0,1, \ldots, m$.

Furthermore, we need the following result.

Lemma 6 (see [36]). Let $v: J \rightarrow H$ be an integrable function such that $v \in \mathscr{P} \mathscr{C}$. Then the function $v(t)=\int_{0}^{t} C(t-s) v(s) d s$ belongs to $\mathscr{P} \mathscr{C}^{1}$, the function $s \rightarrow A S(t-s) \nu(s)$ is integrable on $[0, t], t \in J$, and $v^{\prime}(t)=v(t)+A \int_{0}^{t} S(t-s) v(s) d s=v(t)+$ $\int_{0}^{t} A S(t-s) v(s) d s, t \in J$.
Let $x_{b}\left(x_{0} ; u\right)$ be the state value of system (1) at terminal time $b$ corresponding to the control $u$ and the initial value $x_{0}=\varphi(t) \in \mathscr{B}$. Introduce the set

$$
\mathscr{B}\left(b, x_{0}\right)=\left\{x_{b}\left(x_{0} ; u\right)(0): u(\cdot) \in L^{p}(J, U)\right\},
$$

which is called the reachable set of system (1) at terminal time $b$, and its closure in $H$ is denoted by $\overline{\mathscr{B}\left(b, x_{0}\right)}$.

Now we give the definitions of mild solutions and approximate controllability for system (1).

Definition 7. An $\mathscr{F}_{t}$-adapted stochastic process $x:(-\infty, b] \rightarrow H$ is called a mild solution of system (1) if $x_{0}=\varphi \in \mathscr{B}, x^{\prime}(0)=\phi,\left.x\right|_{J} \in \mathscr{P} \mathscr{C}^{1}$, and the impulsive conditions $\Delta x\left(t_{k}\right)=I_{k}\left(x_{t_{k}}, x^{\prime}\left(t_{k}\right)\right)$ and $\Delta x^{\prime}\left(t_{k}\right)=J_{k}\left(x_{t_{k}}, x^{\prime}\left(t_{k}\right)\right), k=1, \ldots, m$, are satisfied and

(i) $x(t)$ is adapted to $\mathscr{F}_{t}, t \geq 0$.

(ii) $x(t) \in H$ has càdlàg paths on $t \in J$ a.s. and, for each $t \in J, x(t)$ satisfies the integral equation

$$
\begin{aligned}
x(t)= & C(t) \varphi(0)+S(t)[\phi-g(0, \varphi, \phi)] \\
& +\int_{0}^{t} C(t-s) g\left(s, x_{s}, x^{\prime}(s)\right) d s \\
& +\int_{0}^{t} S(t-s) B u(s) d s \\
& +\int_{0}^{t} S(t-s) f(s) d w(s) \\
& +\sum_{0<t_{k}<t} C\left(t-t_{k}\right) I_{k}\left(x_{t_{k}}, x^{\prime}\left(t_{k}\right)\right) \\
& +\sum_{0<t_{k}<t} S\left(t-t_{k}\right) J_{k}\left(x_{t_{k}}, x^{\prime}\left(t_{k}\right)\right), \quad t \in J,
\end{aligned}
$$

where $f \in S_{F, x}=\left\{f \in L^{p}(K, H): f(t) \in\right.$ $F\left(t, x_{t}, x^{\prime}(t), \int_{0}^{t} h\left(t, s, x_{s}, x^{\prime}(s)\right) d s\right)$ a.e. $\left.t \in J\right\}$.

Definition 8. System (1) is said to be approximately controllable on the interval $J$ if $\overline{\mathscr{B}\left(b, x_{0}\right)}=H$.

It is convenient at this point to define operators

$$
\Gamma_{\tau}^{b}=\int_{\tau}^{b} \mathcal{S}_{\alpha}(b-s) B B^{*} s^{*}(b-s) d s
$$$$
0 \leq \tau<b
$$

$$
\begin{aligned}
\Gamma_{0}^{b} & =\int_{0}^{b} S(b-s) B B^{*} S^{*}(b-s) d s \\
R\left(a, \Gamma_{0}^{b}\right) & =\left(a I+\Gamma_{0}^{b}\right)^{-1} \quad \text { for } a>0
\end{aligned}
$$

where $B^{*}$ denotes the adjoint of $B$ and $S^{*}(t)$ is the adjoint of $S(t)$. It is straightforward that the operator $\Gamma_{0}^{b}$ is a linear bounded operator: 
(S1) $a R\left(a, \Gamma_{\tau}^{b}\right) \rightarrow 0,0 \leq \tau<s \leq b$, as $a \rightarrow 0^{+}$in the strong operator topology.

Lemma 9. Assumption (S1) holds if and only if $a R\left(a, \Gamma_{\tau}^{b}\right) \rightarrow$ $0,0 \leq \tau<s \leq b$, as $a \rightarrow 0^{+}$in the strong operator topology.

The proof of this lemma is a straightforward adaptation of the proof of [9, Theorem 2].

Lemma 10 (see [10]). For any $\tilde{x}_{b} \in L^{p}\left(\mathscr{F}_{b}, H\right)$ there exists $\tilde{\phi} \in L_{\mathscr{F}}^{p}\left(\Omega ; L^{2}\left(0, b ; L_{2}^{0}\right)\right)$ such that $\tilde{x}_{b}=E \tilde{x}_{b}+\int_{0}^{b} \tilde{\phi}(s) d w(s)$.

Now for any $a>0$ and $\tilde{x}_{b} \in L^{p}\left(\mathscr{F}_{b}, H\right)$ we define the control function

$$
\begin{aligned}
& u_{x}^{a}(t)=B^{*} S^{*}(b-t)\left(a I+\Gamma_{0}^{b}\right)^{-1}\left[E \tilde{x}_{b}\right. \\
& +\int_{0}^{b} \tilde{\phi}(s) d w(s)-C(b) \varphi(0) \\
& -S(b)[\phi-g(0, \varphi, \phi)]]-B^{*} S^{*}(b-t) \\
& \cdot \int_{0}^{b}\left(a I+\Gamma_{s}^{b}\right)^{-1} C(b-s) g\left(s, x_{s}, x^{\prime}(s)\right) d s \\
& -B^{*} S^{*}(b-t) \int_{0}^{b}\left(a I+\Gamma_{s}^{b}\right)^{-1} S(b-s) f(s) d w(s) \\
& -B^{*} S^{*}(b-t)\left(a I+\Gamma_{s}^{b}\right)^{-1} \\
& +\sum_{k=1}^{m} C\left(b-t_{k}\right) I_{k}\left(x_{t_{k}}, x^{\prime}\left(t_{k}\right)\right)-B^{*} S^{*}(b-t)(a I \\
& \left.+\Gamma_{s}^{b}\right)^{-1} \sum_{k=1}^{m} S\left(b-t_{k}\right) J_{k}\left(x_{t_{k}}, x^{\prime}\left(t_{k}\right)\right),
\end{aligned}
$$

where $f \in S_{F, x}=\left\{f \in L^{p}(K, H): f(t) \in F\left(t, x_{t}, x^{\prime}(t)\right.\right.$, $\left.\int_{0}^{t} h\left(t, s, x_{s}, x^{\prime}(s)\right) d s\right)$ a.e. $\left.t \in J\right\}$. axioms.

The next result is a consequence of the phase space

Lemma 11. Let $x:(-\infty, b] \rightarrow H$ be an $\mathscr{F}_{t}$-adapted measurable process such that $\mathscr{F}_{0}$-adapted process $x_{0}=\varphi(t) \epsilon$ $L_{2}^{0}(\Omega, \mathscr{B})$ and $\left.x\right|_{J} \in \mathscr{P} \mathscr{C}^{1}(J, H)$; then

$$
\left\|x_{s}\right\|_{\mathscr{B}} \leq M_{b} E\|\varphi\|_{\mathscr{B}}+K_{b} \sup _{0 \leq s \leq b} E\|x(s)\|_{H} .
$$

Lemma 12 (see [37]). For any $p \geq 1$ and for arbitrary $L_{2}^{0}(K, H)$-valued predictable process $\phi(\cdot)$,

$$
\begin{aligned}
& \sup _{s \in[0, t]} E\left\|\int_{0}^{s} \phi(v) d w(v)\right\|_{H}^{2 p} \\
& \leq(p(2 p-1))^{p}\left(\int_{0}^{t}\left(E\|\phi(s)\|_{L_{2}^{0}}^{2 p}\right)^{1 / p} d s\right)^{p}, \\
& t \in[0, \infty) .
\end{aligned}
$$

The consideration of this paper is based on the following fixed point theorem due to Dhage [28].

Lemma 13. Let $H$ be a Hilbert space, and let $\Phi_{1}: H \rightarrow$ $\mathscr{P}_{\mathrm{cl}, \mathrm{cv}, \mathrm{bd}}(H)$ and $\Phi_{2}: H \rightarrow \mathscr{P}_{\mathrm{cp}, \mathrm{cv}}(H)$ be two multivalued operators satisfying that
(a) $\Phi_{1}$ is a contraction
(b) $\Phi_{2}$ is completely continuous.

Then either

(i) the operator inclusion $x \in \Phi_{1} x+\Phi_{1} x$ has a solution or

(ii) the set $G=\left\{x \in H: x \in \lambda \Phi_{1} x+\lambda \Phi_{2} x\right\}$ is unbounded for $\lambda \in(0,1)$.

\section{Existence of Solutions for Impulsive Stochastic Control System}

In this section, we prove the existence of solutions for impulsive stochastic control system (1). We make the following hypotheses:

(H1) $A$ is the infinitesimal generator of a strongly continuous cosine family $\{C(t): t \geq 0\}$ on $H$ and the corresponding sine family $\{S(t): t \geq 0\}$ satisfies the conditions $\|C(t)\|_{H} \leq M e^{-\alpha t},\|S(t)\|_{H} \leq M e^{-\beta t}$, and $\|A S(t)\|_{H} \leq M e^{-\gamma t}, t \geq 0$ for some constants $M, \alpha, \beta$, and $\gamma$.

(H2) $C(t), t>0$, is compact.

(H3) The function $g: J \times \mathscr{B} \times H \rightarrow H$ is continuous and there exist $L_{g}>0$ such that

$$
\begin{aligned}
& E\left\|g\left(t_{1}, \psi_{1}, y_{1}\right)-g\left(t_{2}, \psi_{2}, y_{2}\right)\right\|_{H}^{p} \\
& \quad \leq L_{g}\left[\left|t_{1}-t_{2}\right|+\left\|\psi_{1}-\psi_{2}\right\|_{\mathscr{B}}^{p}+E\left\|y_{1}-y_{2}\right\|_{H}^{p}\right]
\end{aligned}
$$

for all $t_{i} \in J, \psi_{i} \in \mathscr{B}, y_{i} \in H, i=1,2$, and

$$
\begin{aligned}
E\|g(t, \psi, y)\|_{H}^{p} \leq L_{g}\left(\|\psi\|_{\mathscr{B}}^{p}+E\|y\|_{H}^{p}+1\right), & \\
& t \in J, \psi \in \mathscr{B}, y \in H,
\end{aligned}
$$

with $\quad L_{g}\left\{M^{p} b^{p-1}(p \alpha)^{-1}+2^{p-1}[1 \quad+\right.$ $\left.\left.M^{2 p} b^{p-1}(p \gamma)^{-1}\right]\right\}\left(2^{p-1} K_{b}^{p}+1\right)<1$.

(H4) The function $h: J \times J \times \mathscr{B} \times H \rightarrow H$ satisfies the following conditions:

(i) For each $(t, s) \in J \times J$ the function $h(t, s, \cdot, \cdot)$ : $\mathscr{B} \times H \rightarrow H$ is continuous and, for each $(\psi, y) \in \mathscr{B} \times H$, the function $h(\cdot, \cdot, \psi, y): J \times$ $J \rightarrow H$ is strongly measurable.

(ii) There exists a continuous function $m_{h}: J \times J \rightarrow$ $[0, \infty)$, such that

$$
E\|h(t, s, \psi, y)\|_{H}^{p} \leq m_{h}(t, s) \Theta_{h}\left(\|\psi\|_{\mathscr{B}}^{p}+\|y\|_{H}^{p}\right)
$$


for a.e. $t, s \in J,(\psi, y) \in \mathscr{B} \times H$, where $\Theta_{h}:[0, \infty) \rightarrow(0, \infty)$ is a continuous nondecreasing function.

(H5) The multivalued map $F: J \times \mathscr{B} \times H \times H \rightarrow$ $\mathscr{P}_{\text {bd,cl,cv }}(L(K, H))$ satisfies the following conditions:

(i) For each $t \in J$, the function $F(t, \cdot, \cdot, \cdot): \mathscr{B} \times H \times$ $H \rightarrow \mathscr{P}_{\text {bd,cl,cv }}(L(K, H))$ is u.s.c. and, for each $(\psi, y, z) \in \mathscr{B} \times H \times H$, the function $F(\cdot, \cdot, \cdot)$ is measurable and the set

$$
\begin{aligned}
& S_{F, \psi}=\left\{f \in L^{p}(J, L(K, H)): f(t)\right. \\
& \in F\left(t, \psi_{t}, \psi^{\prime}(t), \int_{0}^{t} h\left(t, s, \psi_{s}, \psi^{\prime}(s)\right) d s\right) \\
& \quad \text { for a.e. } t \in J\}
\end{aligned}
$$

is nonempty.

(ii) There exist a continuous function $m_{f}: J \rightarrow$ $[0, \infty)$ and a continuous nondecreasing function $\Theta_{f}:[0, \infty) \rightarrow(0, \infty)$ such that

$$
\begin{aligned}
& \|F(t, \psi, y, z)\|_{H}^{p} \\
& \quad \leq m_{f}(t) \Theta_{f}\left(\|\psi\|_{\mathscr{B}}^{p}+E\|y\|_{H}^{p}+E\|z\|_{H}^{p}\right),
\end{aligned}
$$

a.e. $t \in J, \psi \in \mathscr{B}, y, z \in H$, with

$$
\int_{1}^{\infty} \frac{1}{1+s+\Theta_{h}(s)+\Theta_{f}(s)} d s=\infty
$$

where

$$
\|F(t, \psi, y, z)\|_{H}^{p}=\sup \left\{E\|f\|_{H}^{p}: f \in F(t, \psi, y, z)\right\} .
$$

(H6) The functions $I_{k}, J_{k}: \mathscr{B} \times H \rightarrow H$ are continuous and there are constants $c_{k}, d_{k}, k=1,2, \ldots, m, j=1,2,3,4$ such that

$$
\begin{aligned}
0 \leq \lim _{\|\psi\|_{\mathscr{B}}^{p}+\|y\|_{H}^{p} \rightarrow \infty} \frac{E\left\|I_{k}(\psi, y)\right\|_{H}^{p}}{\|\psi\|_{\mathscr{B}}^{p}+E\|y\|_{H}^{p}} \leq c_{k}, \\
0 \leq \lim _{\|\psi\|_{\mathscr{B}}^{p}+E\|y\|_{H}^{p} \rightarrow \infty} \frac{E\left\|J_{k}(\psi, y)\right\|_{H}^{p}}{\|\psi\|_{\mathscr{B}}^{p}+E\|y\|_{H}^{p}} \leq d_{k}
\end{aligned}
$$

for every $\psi \in \mathscr{B}, y \in H$.

Lemma 14 (see [38]). Let $J$ be a compact interval and let $H$ be a Hilbert space. Let $F$ be a multivalued map satisfying (H5) (i) and let $\Gamma$ be a linear continuous operator from $L^{p}(J, H)$ to $C(J, H)$. Then the operator $\Gamma \circ S_{F}: C(J, H) \rightarrow P_{\mathrm{cp}, \mathrm{cv}}(C(J, H))$ is a closed graph in $C(J, H) \times C(J, H)$.

Remark 15. In what follows, we set $M_{1}=\|B\|_{H}, M_{*}=$ $M \max \left\{1, e^{-\alpha b}, e^{-\beta b}, e^{-\gamma b}\right\}$, and $N_{*}=\max \left\{1, e^{\alpha b}, e^{\beta b}, e^{\gamma b}\right\}$.

Theorem 16. If assumptions (H1)-(H6) are satisfied, further, suppose that, for all $a>0$, system (1) has at least one mild solution on $J$, provided that

$$
\begin{aligned}
& {\left[12^{p-1} L_{g}\right.} \\
& \left.\quad+\left(10^{p-1} K_{b}^{p}+12^{p-1}\right) m^{p-1} M^{2 p} \sum_{k=1}^{m}\left(c_{k}+d_{k}\right)\right] N_{*}^{p}
\end{aligned}
$$

$<1$.

Proof. Let $\mathscr{B}_{b}^{0}=\left\{x:(-\infty, b] \rightarrow H ; x_{0}=0,\left.x\right|_{J} \in\right.$ $\left.\mathscr{P} \mathscr{C}^{1}, x^{\prime}(0)=g(0, \varphi, \phi)\right\}$ endowed with the norm of $\mathscr{P} \mathscr{C}^{1}$. Thus $\left(\mathscr{B}_{b}^{0},\|\cdot\|_{\mathscr{P}_{\mathscr{C}^{1}}}\right)$ is Banach space. Now we can define the multivalued map $\Phi: \mathscr{B}_{b}^{0} \rightarrow \mathscr{P}\left(\mathscr{B}_{b}^{0}\right)$ by $\Phi x$ the set of $\rho \in \mathscr{B}_{b}^{0}$ such that

$$
\begin{aligned}
& \rho(t) \\
& = \begin{cases}0, & t \in(-\infty, 0], \\
\int_{0}^{t} C(t-s) g\left(s, \bar{x}_{s}, \bar{x}^{\prime}(s)\right) d s+\int_{0}^{t} S(t-s) B u_{\bar{x}}(s) d s+\int_{0}^{t} S(t-s) f(s) d w(s)+\sum_{0<t_{k}<t} C\left(t-t_{k}\right) I_{k}\left(\bar{x}_{t_{k}}, \bar{x}^{\prime}\left(t_{k}\right)\right)+\sum_{0<t_{k}<t} S\left(t-t_{k}\right) J_{k}\left(\bar{x}_{t_{k}}, \bar{x}^{\prime}\left(t_{k}\right)\right), & t \in J,\end{cases}
\end{aligned}
$$

where

$$
\begin{aligned}
& -B^{*} S^{*}(b-t) \int_{0}^{b}\left(a I+\Gamma_{s}^{b}\right)^{-1} S(b-s) f(s) d w(s) \\
& -B^{*} S^{*}(b-t)\left(a I+\Gamma_{s}^{b}\right)^{-1} \\
& \cdot \sum_{k=1}^{m} C\left(b-t_{k}\right) I_{k}\left(\bar{x}_{t_{k}}, \bar{x}^{\prime}\left(t_{k}\right)\right)-B^{*} S^{*}(b-t)(a I \\
& \left.+\Gamma_{s}^{b}\right)^{-1} \sum_{k=1}^{m} S\left(b-t_{k}\right) J_{k}\left(\bar{x}_{t_{k}}, \bar{x}^{\prime}\left(t_{k}\right)\right),
\end{aligned}
$$$$
u_{x}^{a}(t)=B^{*} S^{*}(b-t)\left(a I+\Gamma_{0}^{b}\right)^{-1}\left[E \tilde{x}_{b}\right.
$$$$
+\int_{0}^{b} \tilde{\phi}(s) d w(s)-C(b) \varphi(0)
$$$$
-S(b)[\phi-g(0, \varphi, \phi)]]-B^{*} S^{*}(b-t)
$$$$
\cdot \int_{0}^{b}\left(a I+\Gamma_{s}^{b}\right)^{-1} C(b-s) g\left(s, \bar{x}_{s}, \bar{x}^{\prime}(s)\right) d s
$$ 
and $f \in S_{F, \bar{x}}=\left\{f \in L^{p}(L(K, H)): f(t) \quad \epsilon\right.$ $F\left(t, \bar{x}_{t}, \bar{x}^{\prime}(t), \int_{0}^{t} h\left(t, s, \bar{x}_{s}, \bar{x}^{\prime}(s)\right) d s\right)$ a.e. $\left.t \in J\right\}$, and $\bar{x}:$ $(-\infty, 0] \rightarrow H$ is such that $\bar{x}_{0}=\varphi$ and $\bar{x}=x$ on $J$. In what follows, we aim to show that the operator $\Phi$ has a fixed point, which is a solution of problem (1).

Let $\left\{\sigma_{n}: n \in \mathbb{N}\right\}$ be a decreasing sequence in $\left(0, t_{1}\right) \subset(0, b)$ such that $\lim _{n \rightarrow \infty} \sigma_{n}=0$. To prove the above theorem, we consider the following problem:

$$
\begin{aligned}
& d\left[x^{\prime}(t)-g\left(t, x_{t}, x^{\prime}(t)\right)\right] \\
& \quad \in[A x(t)+B u(t)] d t \\
& \quad+F\left(t, x_{t}, x^{\prime}(t), \int_{0}^{t} h\left(t, s, x_{s}, x^{\prime}(s)\right) d s\right) d w(t),
\end{aligned}
$$

$t \in J, t \neq t_{k}$,

$$
\begin{aligned}
& \Delta x\left(t_{k}\right)=C\left(\sigma_{n}\right) I_{k}\left(x\left(t_{k}\right), x^{\prime}\left(t_{k}\right)\right), \quad k=1, \ldots, m, \\
& \Delta x^{\prime}\left(t_{k}\right)=S\left(\sigma_{n}\right) J_{k}\left(x\left(t_{k}\right), x^{\prime}\left(t_{k}\right)\right), \quad k=1, \ldots, m, \\
& x_{0}=\varphi \in \mathscr{B}, \\
& x^{\prime}(0)=\phi .
\end{aligned}
$$

We will show that the problem has at least one mild solution $x_{n} \in \mathscr{B}_{b}^{0}$.

For fixed $n \in \mathbb{N}$, set the multivalued map $\Phi_{n}: \mathscr{B}_{b}^{0} \rightarrow$ $\mathscr{P}\left(\mathscr{B}_{b}^{0}\right)$ by $\Phi_{n} x$ the set of $\rho_{n} \in \mathscr{B}_{b}^{0}$ such that

$$
\begin{aligned}
& \rho_{n}(t) \\
& = \begin{cases}0, & t \in(-\infty, 0], \\
\int_{0}^{t} C(t-s) g\left(s, \bar{x}_{s}, \bar{x}^{\prime}(s)\right) d s+\int_{0}^{t} S(t-s) B u_{\bar{x}}^{a}(s) d s+\int_{0}^{t} S(t-s) f(s) d w(s)+\sum_{0<t_{k}<t} C\left(t-t_{k}\right) C\left(\sigma_{n}\right) I_{k}\left(\bar{x}_{t_{k}}, \bar{x}^{\prime}\left(t_{k}\right)\right)+\sum_{0<t_{k}<t} S\left(t-t_{k}\right) S\left(\sigma_{n}\right) J_{k}\left(\bar{x}_{t_{k}}, \bar{x}^{\prime}\left(t_{k}\right)\right), & t \in J,\end{cases}
\end{aligned}
$$

where

$$
\begin{aligned}
& u_{\bar{x}}^{a}(t)=B^{*} S^{*}(b-t)\left(a I+\Gamma_{0}^{b}\right)^{-1}\left[E \widetilde{x}_{b}\right. \\
& +\int_{0}^{b} \tilde{\phi}(s) d w(s)-C(b) \varphi(0) \\
& \quad-S(b)[\phi-g(0, \varphi, \phi)]]-B^{*} S^{*}(b-t) \\
& \cdot \int_{0}^{b}\left(a I+\Gamma_{s}^{b}\right)^{-1} C(b-s) g\left(s, \bar{x}_{s}, \bar{x}^{\prime}(s)\right) d s \\
& -B^{*} S^{*}(b-t) \int_{0}^{b}\left(a I+\Gamma_{s}^{b}\right)^{-1} S(b-s) f(s) d w(s)
\end{aligned}
$$

$$
\begin{aligned}
& -B^{*} S^{*}(b-t)\left(a I+\Gamma_{s}^{b}\right)^{-1} \\
& \cdot \sum_{k=1}^{m} C\left(b-t_{k}\right) C\left(\sigma_{n}\right) I_{k}\left(\bar{x}_{t_{k}}, \bar{x}^{\prime}\left(t_{k}\right)\right)-B^{*} \mathcal{S}_{\alpha}^{*}(b \\
& -t)\left(a I+\Gamma_{s}^{b}\right)^{-1} \sum_{k=1}^{m} S\left(b-t_{k}\right) S\left(\sigma_{n}\right) J_{k}\left(\bar{x}_{t_{k}}, \bar{x}^{\prime}\left(t_{k}\right)\right),
\end{aligned}
$$

and $f \in S_{F, \bar{x}}$. It is easy to see that the fixed point of $\Phi_{n}$ is a mild solution of the Cauchy problem (27).

Let $\bar{\varphi}:(-\infty, 0) \rightarrow H$ be the extension of $(-\infty, 0]$ such that $\bar{\varphi}(\theta)=\varphi(0)=0$ on $J$. Now, we consider the following multivalued operators $\Lambda_{n}$ and $\Upsilon_{n}$ defined by

$$
\left(\Lambda_{n} x\right)(t)= \begin{cases}0, & t \in(-\infty, 0], \\ \int_{0}^{t} C(t-s) g\left(s, \bar{x}_{s}, \bar{x}^{\prime}(s)\right) d s, & t \in J\end{cases}
$$

$\left(\Upsilon_{n} x\right)(t)$

$$
=\left\{\begin{array}{lll}
0 & t \in(-\infty, 0], \\
\int_{0}^{t} S(t-s) B u_{\bar{x}}(s) d s+\int_{0}^{t} S(t-s) f(s) d w(s)+\sum_{0<t_{k}<t} C\left(t-t_{k}\right) C\left(\sigma_{n}\right) I_{k}\left(\bar{x}_{t_{k}}, \bar{x}^{\prime}\left(t_{k}\right)\right)+\sum_{0<t_{k}<t} S\left(t-t_{k}\right) S\left(\sigma_{n}\right) J_{k}\left(\bar{x}_{t_{k}}, \bar{x}^{\prime}\left(t_{k}\right)\right) & t \in J .
\end{array}\right.
$$

It is clear that $\Phi_{n}=\Lambda_{n}+\Upsilon_{n}$. The problem of finding mild solutions of (27) is reduced to find the solutions of the operator inclusion $x \in \Lambda_{n}(x)+\Upsilon_{n}(x)$. In what follows, we show that operators $\Lambda_{n}$ and $\Upsilon_{n}$ satisfy the conditions of Lemma 13.

Step 1. $\Lambda_{n}$ is a contraction on $\mathscr{B}_{b}^{0}$.
Let $t \in J$ and $x^{*}, x^{* *} \in \mathscr{B}_{b}^{0}$. From (H3) and Lemma 11, we have

$$
\begin{gathered}
E\left\|\left(\Lambda_{n} x^{*}\right)(t)-\left(\Lambda_{n} x^{* *}\right)(t)\right\|_{H}^{p}=E \| \int_{0}^{t} C(t-s) \\
\cdot\left[g\left(s,\left(\overline{x^{*}}\right)_{t},\left(\overline{x^{*}}\right)^{\prime}(s)\right)\right.
\end{gathered}
$$




$$
\begin{aligned}
& \left.-g\left(s,\left(\overline{x^{* *}}\right)_{t},\left(\overline{x^{* *}}\right)^{\prime}(s)\right)\right] d s \|_{H}^{p} \\
& \leq M^{p} E\left[\int_{0}^{t} e^{-\alpha(t-s)} \| g\left(s,\left(\overline{x^{*}}\right)_{t},\left(\overline{x^{*}}\right)^{\prime}(s)\right)\right. \\
& \left.-g\left(s,\left(\overline{x^{* *}}\right)_{t},\left(\overline{x^{* *}}\right)^{\prime}(s)\right) \|_{H} d s\right]^{p} \\
& \leq M^{p} L_{g} b^{p-1} \int_{0}^{t} e^{-p \alpha(t-s)}\left[\left\|\left(\overline{x^{*}}\right)_{s}-\left(\overline{x^{* *}}\right)_{s}\right\|_{\mathscr{B}}^{p}\right. \\
& \left.+E\left\|\left(\overline{x^{*}}\right)^{\prime}(s)-\left(\overline{x^{* *}}\right)^{\prime}(s)\right\|_{H}^{p}\right] d s \\
& \leq M^{p} L_{g} b^{p-1} \int_{0}^{t} e^{-p \alpha(t-s)} d s\left[2^{p-1} K_{b}^{p} \sup _{s \in[0, b]} \|_{x^{*}}(s)\right. \\
& \left.\leq \overline{x^{* *}}(s)\left\|_{H}^{p}+\sup _{s \in[0, b]} E\right\|\left(\overline{x^{*}}\right)^{\prime}(s)-\left(\overline{x^{* *}}\right)^{\prime}(s) \|_{H}^{p}\right] \\
& \left.\leq x_{g}^{* *}(s)\left\|_{H}^{p}+\sup _{s \in[0, b]} E\right\|\left(x^{*}\right)^{\prime}(s)-\left(x^{* *}\right)^{\prime}(s) \|_{H}^{p}\right] \\
& \leq b^{p-1}(p \alpha)^{-1}\left[2^{p-1} K_{b}^{p} \sup _{s \in[0, b]} E x^{*}(s)\right.
\end{aligned}
$$

By Lemma 6, we have

$$
\begin{aligned}
\Lambda_{n}^{\prime}(t)= & g\left(t, \bar{x}_{t}, \bar{x}^{\prime}(t)\right) \\
& +\int_{0}^{t} A S(t-s) g\left(s, \bar{x}_{s}, \bar{x}^{\prime}(s)\right) d s, \quad t \in J .
\end{aligned}
$$

Similarly, for any $t \in[0, b]$, we have

$$
\begin{aligned}
E & \left\|\left(\Lambda_{n}^{\prime} x^{*}\right)(t)-\left(\Lambda_{n}^{\prime} x^{* *}\right)(t)\right\|_{H}^{p} \leq 2^{p-1} E \| g(t, \\
& \left.\left(\overline{x^{*}}\right)_{t},\left(\overline{x^{*}}\right)^{\prime}(t)\right)-g\left(t,\left(\overline{x^{* *}}\right)_{t},\left(\overline{x^{* *}}\right)^{\prime}(t)\right) \|_{H}^{p} \\
& +2^{p-1} E \| \int_{0}^{t} A S(t-s)\left[g\left(s,\left(\overline{x^{*}}\right)_{s},\left(\overline{x^{*}}\right)^{\prime}(s)\right)\right. \\
& \left.-g\left(s,\left(\overline{x^{* *}}\right)_{s},\left(\overline{x^{* *}}\right)^{\prime}(s)\right)\right] d s \|_{H}^{p} \\
& \leq 2^{p-1}\left[\left\|\left(\overline{x^{*}}\right)_{t}-\left(\overline{x^{* *}}\right)_{t}\right\|_{\mathscr{B}}^{p}+E \|\left(\overline{x^{*}}\right)^{\prime}(t)\right. \\
& \left.-\left(\overline{x^{* *}}\right)^{\prime}(t) \|_{H}^{p}\right] \\
& +2^{p-1} M^{p} L_{g} b^{p-1} \int_{0}^{t} e^{-p \gamma(t-s)}\left[\left\|\left(\overline{x^{*}}\right)_{s}-\left(\overline{x^{* *}}\right)_{s}\right\|_{\mathscr{B}}^{p}\right.
\end{aligned}
$$

$$
\begin{aligned}
& \left.+E\left\|\left(\overline{x^{*}}\right)^{\prime}(s)-\left(\overline{x^{* *}}\right)^{\prime}(s)\right\|_{H}^{p}\right] d s \\
& \leq 2^{p-1} L_{g}\left[2^{p-1} K_{b}^{p} \sup _{t \in[0, b]} E\left\|\overline{x^{*}}(t)-\overline{x^{* *}}(t)\right\|_{H}^{p}\right. \\
& \left.+\sup _{t \in[0, b]} E\left\|\left(\overline{x^{*}}\right)^{\prime}(t)-\left(\overline{x^{* *}}\right)^{\prime}(t)\right\|_{H}^{p}\right] \\
& +2^{p-1} M^{p} L_{g} b^{p-1} \int_{0}^{t} e^{-p \gamma(t-s)} d s\left[2^{p-1} K_{b}^{p}\right. \\
& \text {. } \sup _{s \in[0, b]} E\left\|\overline{x^{*}}(s)-\overline{x^{* *}}(s)\right\|_{H}^{p}+\sup _{s \in[0, b]} E \|\left(\overline{x^{*}}\right)^{\prime}(s) \\
& \left.-\left(\overline{x^{* *}}\right)^{\prime}(s) \|_{H}^{p}\right] \leq\left[2^{p-1} L_{g}\right. \\
& \left.+2^{p-1} M^{2 p} L_{g} e^{-\alpha \sigma_{n}} b^{p-1}(p \gamma)^{-1}\right]\left[2^{p-1} K_{b}^{p}\right. \\
& \text {. } \sup _{s \in[0, b]} E\left\|x^{*}(s)-x^{* *}(s)\right\|_{H}^{p}+\sup _{s \in[0, b]} E \|\left(x^{*}\right)^{\prime}(s) \\
& \left.-\left(x^{* *}\right)^{\prime}(s) \|_{H}^{p}\right] \quad(\text { since } \bar{x}=x \text { on } J) \\
& \leq 2^{p^{-1}} L_{g}\left[1+M^{p} b^{p-1}(p \gamma)^{-1}\right]\left(2^{p-1} K_{b}^{p}+1\right) \| x^{*} \\
& -x^{* *} \|_{\mathscr{P} \mathscr{C}^{1}}^{p} \text {. }
\end{aligned}
$$

Taking supremum over $t$, it follows that

$$
\left\|\Lambda_{n} x^{*}-\Lambda_{n}^{* *}\right\|_{\mathscr{P} \mathscr{C}^{1}}^{p} \leq L_{0}\left\|x^{*}-x^{* *}\right\|_{\mathscr{P} \mathscr{C}^{1}}^{p}
$$

where $L_{0}=L_{g}\left\{M^{p} b^{p-1}(p \alpha)^{-1}+2^{p-1}[1+\right.$ $\left.\left.M^{2 p} b^{p-1}(p \gamma)^{-1}\right]\right\}\left(2^{p-1} K_{b}^{p}+1\right)<1$. Hence, $\Lambda_{n}$ is a contraction on $\mathscr{B}_{b}^{0}$.

Step 2. $\Upsilon_{n}$ has compact, convex values and it is completely continuous.

(1) $\Upsilon_{n} x$ is convex for each $x \in \mathscr{B}_{b}^{0}$.

In fact, if $\tilde{\rho}_{n}^{1}, \widetilde{\rho}_{n}^{2}$ belong to $\Upsilon_{n} x$, then there exist $f_{1}, f_{2} \in$ $S_{F, \bar{x}}$ such that

$$
\begin{aligned}
& \tilde{\rho}_{n}^{i}(t)=\int_{0}^{t} S(t-s) B\left\{B ^ { * } S ^ { * } ( b - s ) ( a I + \Gamma _ { 0 } ^ { b } ) ^ { - 1 } \left[E \widetilde{x}_{b}\right.\right. \\
& +\int_{0}^{b} \tilde{\phi}(\eta) d w(\eta)-C(b) \varphi(0) \\
& -S(b)[\phi-g(0, \varphi, \phi)]]-B^{*} S^{*}(b-s) \\
& \cdot \int_{0}^{b}\left(a I+\Gamma_{\eta}^{b}\right)^{-1} C(b-\eta) g\left(\eta, \bar{x}_{\eta}, \bar{x}^{\prime}(\eta)\right) d \eta
\end{aligned}
$$




$$
\begin{aligned}
& -B^{*} S^{*}(b-s) \\
& \cdot \int_{0}^{b}\left(a I+\Gamma_{\eta}^{b}\right)^{-1} S(b-\eta) f_{i}(\eta) d w(\eta) \\
& -B^{*} S^{*}(b-s)\left(a I+\Gamma_{\eta}^{b}\right)^{-1} \\
& \cdot \sum_{k=1}^{m} C\left(b-t_{k}\right) C\left(\sigma_{n}\right) I_{k}\left(\bar{x}_{t_{k}}, \bar{x}\left(t_{k}\right)\right)-B^{*} S^{*}(b \\
& -s)\left(a I+\Gamma_{\eta}^{b}\right)^{-1} \\
& \left.\cdot \sum_{k=1}^{m} S\left(b-t_{k}\right) S\left(\sigma_{n}\right) J_{k}\left(\bar{x}_{t_{k}}, \bar{x}^{\prime}\left(t_{k}\right)\right)\right\} d s \\
& +\int_{0}^{t} S(t-s) f_{i}(s) d w(s)+\sum_{0<t_{k}<t} C\left(t-t_{k}\right) \\
& \cdot C\left(\sigma_{n}\right) I_{k}\left(\bar{x}_{t_{k}}, \bar{x}^{\prime}\left(t_{k}\right)\right)+\sum_{0<t_{k}<t} S\left(t-t_{k}\right) S\left(\sigma_{n}\right) \\
& \cdot J_{k}\left(\bar{x}_{t_{k}}, \bar{x}^{\prime}\left(t_{k}\right)\right), \quad t \in[0, b], i=1,2 .
\end{aligned}
$$

Let $0 \leq \lambda \leq 1$. For each $t \in[0, b]$ we have

$$
\begin{aligned}
& \left(\lambda \widetilde{\rho}_{n}^{1}+(1-\lambda) \tilde{\rho}_{n}^{2}\right)(t)=\int_{0}^{t} S(t-s) B\left\{B^{*} S^{*}(b-s)\right. \\
& \cdot\left(a I+\Gamma_{0}^{b}\right)^{-1}\left[E \tilde{x}_{b}+\int_{0}^{b} \tilde{\phi}(\eta) d w(\eta)-C(b) \varphi(0)\right. \\
& -S(b)[\phi-g(0, \varphi, \phi)]]-B^{*} \mathcal{S}_{\alpha}^{*}(b-s) \\
& \cdot \int_{0}^{b}\left(a I+\Gamma_{\eta}^{b}\right)^{-1} C(b-\eta) g\left(\eta, \bar{x}_{\eta}, \bar{x}^{\prime}(\eta)\right) d \eta \\
& -B^{*} S_{\alpha}^{*}(b-s) \int_{0}^{b}\left(a I+\Gamma_{\eta}^{b}\right)^{-1} S(b-\eta) \\
& \cdot\left[\lambda f_{1}(\eta)+(1-\lambda) f_{2}(\eta)\right] d w(\eta) \\
& -B^{*} S^{*}(b-s)\left(a I+\Gamma_{\eta}^{b}\right)^{-1} \sum_{k=1}^{m} C\left(b-t_{k}\right) C\left(\sigma_{n}\right) \\
& \cdot I_{k}\left(\bar{x}_{t_{k}}, \bar{x}\left(t_{k}\right)\right)-B^{*} S^{*}(b-s)\left(a I+\Gamma_{\eta}^{b}\right)^{-1} \\
& \left.+\sum_{k=1}^{m} S\left(b-t_{k}\right) S\left(\sigma_{n}\right) J_{k}\left(\bar{x}_{t_{k}}, \bar{x}^{\prime}\left(t_{k}\right)\right)\right\} d s \\
& +\int_{0}^{t} S(t-s)\left[\lambda f_{1}(s)+(1-\lambda) f_{2}(s)\right] d w(s) \\
& +\sum_{0<t_{k}<t} C\left(t-t_{k}\right) C\left(\sigma_{n}\right) I_{k}\left(\bar{x}_{t_{k}}, \bar{x}^{\prime}\left(t_{k}\right)\right)+\sum_{0<t_{k}<t} S(t \\
& \left.-t_{k}\right) S\left(\sigma_{n}\right) J_{k}\left(\bar{x}_{t_{k}}, \bar{x}^{\prime}\left(t_{k}\right)\right) .
\end{aligned}
$$

Since $S_{F, \bar{x}}$ is convex (because $F$ has convex values) we have $\left(\lambda \widetilde{\rho}_{n}^{1}+(1-\lambda) \tilde{\rho}_{n}^{2}\right) \in \Upsilon_{n} x$.

(2) $\Upsilon_{n}$ maps bounded sets into bounded sets in $\mathscr{B}_{b}^{0}$.

Indeed, it is enough to show that there exists a positive constant $\mathscr{L}$ such that, for each $\widetilde{\rho}_{n} \in \Upsilon_{n} x, x \in B_{r}\left(0, \mathscr{B}_{b}^{0}\right)=$ $\left\{x \in \mathscr{B}_{b}^{0}:\|x\|_{\mathscr{P} \mathscr{C}^{1}}^{p} \leq r\right\}$, one has $\left\|\widetilde{\rho}_{n}\right\|_{\mathscr{P} \mathscr{C}^{1}}^{p} \leq \mathscr{L}$. If $\widetilde{\rho}_{n} \in \Upsilon_{n} x$, then there exists $f \in S_{F, \bar{x}}$ such that, for each $t \in J$,

$$
\begin{aligned}
\tilde{\rho}_{n}(t)= & \int_{0}^{t} S(t-s) B u_{\bar{x}}^{a}(s) d s \\
& +\int_{0}^{t} S(t-s) f(s) d w(s) \\
& +\sum_{0<t_{k}<t} C\left(t-t_{k}\right) C\left(\sigma_{n}\right) I_{k}\left(\bar{x}_{t_{k}}, \bar{x}^{\prime}\left(t_{k}\right)\right) \\
& +\sum_{0<t_{k}<t} S\left(t-t_{k}\right) S\left(\sigma_{n}\right) J_{k}\left(\bar{x}_{t_{k}}, \bar{x}^{\prime}\left(t_{k}\right)\right) .
\end{aligned}
$$

However, on the other hand, from the condition (H6), we conclude that there exist positive constants $\epsilon_{k}(k=$ $1, \ldots, m), \gamma_{1}$ such that, for all $\|\psi\|_{\mathscr{B}}^{p}+\|y\|_{H}^{p}>\gamma_{1}$,

$$
\begin{gathered}
E\left\|I_{k}(\psi, y)\right\|_{H}^{p} \leq\left(c_{k}+\epsilon_{k}\right)\left[\|\psi\|_{\mathscr{B}}^{p}+E\|y\|_{H}^{p}\right], \\
E\left\|J_{k}(\psi, y)\right\|_{H}^{p} \leq\left(d_{k}+\epsilon_{k}\right)\left[\|\psi\|_{\mathscr{B}}^{p}+E\|y\|_{H}^{p}\right], \\
{\left[12^{p-1} L_{g}+\left(10^{p-1} K_{b}^{p}+12^{p-1}\right)\right.} \\
\left.\cdot m^{p-1} M^{2 p} \sum_{k=1}^{m}\left(c_{k}+d_{k}+2 \epsilon_{k}\right)\right] N_{*}^{p}<1 .
\end{gathered}
$$

Let

$$
\begin{aligned}
& F_{1}=\left\{\psi:\|\psi\|_{\mathscr{B}}^{p}+E\|y\|_{H}^{p} \leq \gamma_{1}\right\}, \\
& F_{2}=\left\{\psi:\|\psi\|_{\mathscr{B}}^{p}+E\|y\|_{H}^{p}>\gamma_{1}\right\}, \\
& C_{1}=\max \left\{\left\|I_{k}(\psi, y)\right\|_{H}^{p}, x \in F_{1}\right\} .
\end{aligned}
$$

Therefore

$$
\begin{aligned}
& E\left\|I_{k}(\psi, y)\right\|_{H}^{p} \leq C_{1}+\left(c_{k}+\epsilon_{k}\right)\left(\|\psi\|_{\mathscr{B}}^{p}+E\|y\|_{H}^{p}\right), \\
& E\left\|J_{k}(\psi, y)\right\|_{H}^{p} \leq C_{1}+\left(d_{k}+\epsilon_{k}\right)\left(\|\psi\|_{\mathscr{B}}^{p}+E\|y\|_{H}^{p}\right) .
\end{aligned}
$$

If $x \in B_{r}\left(0, \mathscr{B}_{b}^{0}\right)$, from Lemma 11, it follows that

$$
\left\|\bar{x}_{s}\right\|_{\mathscr{B}}^{p} \leq 2^{p-1}\left(M_{b}\|\varphi\|_{\mathscr{B}}\right)^{p}+2^{p-1} K_{b}^{p} r:=r^{*} .
$$

By (H1)-(H5) and (40)-(41), from (37) we have for $t \in J$ 


$$
\begin{aligned}
& E\left\|\widetilde{\rho}_{n}\right\|_{H}^{p} \leq 4^{p-1} E \| \int_{0}^{t} S(t-s) B\left\{B^{*} S^{*}(b-s)\left(a I+\Gamma_{0}^{b}\right)^{-1}\left[E \tilde{x}_{b}+\int_{0}^{b} \tilde{\phi}(\eta) d w(\eta)-C(b) \varphi(0)-S(b)[\phi-g(0, \varphi, \phi)]\right]\right. \\
& -B^{*} S^{*}(b-s) \int_{0}^{b}\left(a I+\Gamma_{\eta}^{b}\right)^{-1} C(b-\eta) g\left(\eta, \bar{x}_{\eta}, \bar{x}^{\prime}(\eta)\right) d \eta-B^{*} S^{*}(b-s) \int_{0}^{b}\left(a I+\Gamma_{\eta}^{b}\right)^{-1} S(b-\eta) f_{i}(\eta) d w(\eta) \\
& -B^{*} S^{*}(b-s)\left(a I+\Gamma_{\eta}^{b}\right)^{-1} \sum_{k=1}^{m} C\left(b-t_{k}\right) C\left(\sigma_{n}\right) I_{k}\left(\bar{x}_{t_{k}}, \bar{x}\left(t_{k}\right)\right) \\
& \left.-B^{*} S^{*}(b-s)\left(a I+\Gamma_{\eta}^{b}\right)^{-1} \sum_{k=1}^{m} S\left(b-t_{k}\right) S\left(\sigma_{n}\right) J_{k}\left(\bar{x}_{t_{k}}, \bar{x}^{\prime}\left(t_{k}\right)\right)\right\} d s\left\|_{H}^{p}+4^{p-1} E\right\| \int_{0}^{t} S(t-s) f(s) d w(s) \|_{H}^{p} \\
& +4^{p-1} E\left\|\sum_{0<t_{k}<t} C\left(t-t_{k}\right) C\left(\sigma_{n}\right) I_{k}\left(\bar{x}_{t_{k}}, \bar{x}^{\prime}\left(t_{k}\right)\right)\right\|_{H}^{p}+4^{p-1} E\left\|\sum_{0<t_{k}<t} S\left(t-t_{k}\right) S\left(\sigma_{n}\right) J_{k}\left(\bar{x}_{t_{k}}, \bar{x}^{\prime}\left(t_{k}\right)\right)\right\|_{H}^{p} \leq 20^{p-1} M^{p} e^{-p \beta t} \\
& \cdot \frac{1}{a^{p}} M^{p} e^{-p \beta b} M_{1}^{2 p} b^{p-1} \int_{0}^{t} e^{2 p \beta s}\left\{4 ^ { p - 1 } \left[\left\|E \tilde{x}_{b}\right\|_{H}^{p}+C_{p}\left[\int_{0}^{b}\left[E\|\widetilde{\phi}(\eta)\|_{H}^{p}\right]^{2 / p} d \eta\right]^{p / 2}\right.\right. \\
& \left.+M^{p} e^{-p \alpha b}\left[E\|\varphi(0)\|_{H}^{p}+2^{p-1}\left[\|\phi\|_{H}^{p}+\|g(0, \varphi, \phi)\|_{H}^{p}\right]\right]\right]+M^{p} b^{p-1} L_{g} \int_{0}^{b} e^{-p \alpha(b-\eta)}\left[\left\|\bar{x}_{\eta}\right\|_{\mathscr{B}}^{p}+E\left\|\bar{x}^{\prime}(\eta)\right\|_{H}^{p}+1\right] d \eta \\
& +C_{p} M^{p}\left[\int_{0}^{b}\left[e^{-p \beta(b-\eta)} E\|f(\eta)\|_{H}^{p}\right]^{2 / p} d \eta\right]^{p / 2}+m^{p-1} M^{2 p} \sum_{k=1}^{m} e^{-p \alpha\left(b-t_{k}\right)} e^{-p \alpha \sigma_{n}} E\left\|I_{k}\left(\bar{x}_{t_{k}}, \bar{x}^{\prime}\left(t_{k}\right)\right)\right\|_{H}^{p} \\
& \left.+m^{p-1} M^{2 p} \sum_{k=1}^{m} e^{-p \beta\left(b-t_{k}\right)} e^{-p \beta \sigma_{n}} E\left\|J_{k}\left(\bar{x}_{t_{k}}, \bar{x}^{\prime}\left(t_{k}\right)\right)\right\|_{H}^{p}\right\} d s+4^{p-1} C_{p} M^{p}\left[\int_{0}^{t}\left[e^{-p \beta(t-s)} E\|f(s)\|_{H}^{p}\right]^{2 / p} d s\right]^{p / 2} \\
& +(4 m)^{p-1} M^{2 p} \sum_{k=1}^{m} e^{-p \alpha\left(t-t_{k}\right)} e^{-p \alpha \sigma_{n}} E\left\|I_{k}\left(\bar{x}_{t_{k}}, \bar{x}^{\prime}\left(t_{k}\right)\right)\right\|_{H}^{p}+(4 m)^{p-1} M^{2 p} \sum_{k=1}^{m} e^{-p \beta\left(t-t_{k}\right)} e^{-p \beta \sigma_{n}} E\left\|J_{k}\left(\bar{x}_{t_{k}}, \bar{x}^{\prime}\left(t_{k}\right)\right)\right\|_{H}^{p} \\
& \leq 20^{p-1} M^{p} e^{-p \beta t} \frac{1}{a^{p}} M^{p} e^{-p \beta b} M_{1}^{2 p} b^{p-1} \int_{0}^{t} e^{2 p \beta s}\left\{4 ^ { p - 1 } \left[\left\|E \tilde{x}_{b}\right\|_{H}^{p}+C_{p} b^{p / 2-1} \int_{0}^{b} E\|\tilde{\phi}(\eta)\|_{H}^{p} d \eta\right.\right. \\
& \left.+M^{p} e^{-p \alpha b}\left[E\|\varphi(0)\|_{H}^{p}+2^{p-1}\left[\|\phi\|_{H}^{p}+\|g(0, \varphi, \phi)\|_{H}^{p}\right]\right]\right]+M^{p} b^{p-1} L_{g} \int_{0}^{b} e^{-p \alpha(t-\eta)}\left[\left\|\bar{x}_{\eta}\right\|_{\mathscr{B}}^{p}+E\left\|\bar{x}^{\prime}(\eta)\right\|_{H}^{p}+1\right] d \eta \\
& +C_{p} M^{p} b^{p / 2-1} \int_{0}^{b} e^{-p \beta(b-\eta)} m_{f}(\eta) \Theta_{f}\left(\left\|\bar{x}_{\eta}\right\|_{\mathscr{B}}^{p}+E\left\|\bar{x}^{\prime}(s)\right\|_{H}^{p}+b^{p-1} \int_{0}^{\eta} m_{h}(\eta, \nu) \Theta_{h}\left(\left\|\bar{x}_{\nu}\right\|_{\mathscr{B}}^{p}+E\left\|\bar{x}^{\prime}(\nu)\right\|_{H}^{p}\right) d \nu\right) d \eta \\
& +m^{p-1} M^{2 p} \sum_{k=1}^{m} e^{-p \alpha\left(b-t_{k}\right)} e^{-p \alpha \sigma_{n}}\left[C_{1}+\left(c_{k}+\epsilon_{k}\right)\left(\left\|\bar{x}_{t_{k}}\right\|_{\mathscr{B}}^{p}+E\left\|\bar{x}^{\prime}\left(t_{k}\right)\right\|_{H}^{p}\right)\right] \\
& \left.+m^{p-1} M^{2 p} \sum_{k=1}^{m} e^{-p \beta\left(b-t_{k}\right)} e^{-p \beta \sigma_{n}}\left[C_{1}+\left(d_{k}+\epsilon_{k}\right)\left(\left\|\bar{x}_{t_{k}}\right\|_{\mathscr{B}}^{p}+E\left\|\bar{x}^{\prime}\left(t_{k}\right)\right\|_{H}^{p}\right)\right]\right\} d s+4^{p-1} C_{p} M^{p} b^{p / 2-1} \int_{0}^{t} e^{-p \beta(t-s)} m_{f}(s) \\
& \cdot \Theta_{f}\left(\left\|\bar{x}_{s}\right\|_{\mathscr{B}}^{p}+E\left\|\bar{x}^{\prime}(s)\right\|_{H}^{p}+b^{p-1} \int_{0}^{s} m_{h}(s, \tau) \Theta_{h}\left(\left\|\bar{x}_{\tau}\right\|_{\mathscr{B}}^{p}+E\left\|\bar{x}^{\prime}(\tau)\right\|_{H}^{p}\right) d \tau\right) d s+(4 m)^{p-1}
\end{aligned}
$$




$$
\begin{aligned}
& \cdot M^{2 p} \sum_{k=1}^{m} e^{-p \alpha\left(t-t_{k}\right)} e^{-p \alpha \sigma_{n}}\left[C_{1}+\left(c_{k}+\epsilon_{k}\right)\left(\left\|\bar{x}_{t_{k}}\right\|_{\mathscr{B}}^{p}+E\left\|\bar{x}^{\prime}\left(t_{k}\right)\right\|_{H}^{p}\right)\right]+(4 m)^{p-1} M^{2 p} \sum_{k=1}^{m} e^{-p \beta\left(t-t_{k}\right)} e^{-p \beta \sigma_{n}}\left[C_{1}+\left(d_{k}+\epsilon_{k}\right)\right. \\
& \left.\cdot\left(\left\|\bar{x}_{t_{k}}\right\|_{B}^{p}+E\left\|\bar{x}^{\prime}\left(t_{k}\right)\right\|_{H}^{p}\right)\right] \leq 20^{p-1} M_{*}^{p} \frac{1}{a^{p}} M^{p} e^{-p \beta b} M_{1}^{2 p} b^{p} N_{*}^{2 p}\left\{4 ^ { p - 1 } \left[\left\|E \tilde{x}_{b}\right\|_{H}^{p}+C_{p} b^{p / 2-1} \int_{0}^{b} E\|\widetilde{\phi}(\eta)\|_{H}^{p} d \eta\right.\right. \\
& \left.+M^{p} e^{-p \alpha b}\left[E\|\varphi(0)\|_{H}^{p}+2^{p-1}\left[\|\phi\|_{H}^{p}+\|g(0, \varphi, \phi)\|_{H}^{p}\right]\right]\right]+M^{p} b^{p-1} L_{g}\left(r^{*}+r+1\right) \int_{0}^{b} e^{-\alpha p(t-\eta)} d \eta \\
& +C_{p} M^{p} b^{p / 2-1} \Theta_{f}\left(r^{* *}\right) \int_{0}^{b} e^{-p \beta(b-\eta)} m_{f}(\eta) d \eta+m^{p-1} M^{2 p} e^{-p \alpha b} e^{-p \alpha \sigma_{n}} N_{*}^{p} \sum_{k=1}^{m}\left[C_{1}+\left(c_{k}+\epsilon_{k}\right)\left(r^{*}+r\right)\right] \\
& \left.+m^{p-1} M^{2 p} e^{-p \beta b} e^{-p \beta \sigma_{n}} N_{*}^{p} \sum_{k=1}^{m}\left[C_{1}+\left(d_{k}+\epsilon_{k}\right)\left(r^{*}+r\right)\right]\right\}+4^{p-1} C_{p} M^{p} b^{p / 2-1} \Theta_{f}\left(r^{* *}\right) \int_{0}^{b} e^{-p \beta(b-s)} m_{f}(s) d s \\
& +(4 m)^{p-1} M_{*}^{p} M^{p} e^{-p \alpha \sigma_{n}} N_{*}^{p} \sum_{k=1}^{m}\left[C_{1}+\left(c_{k}+\epsilon_{k}\right)\left(r^{*}+r\right)\right]+(4 m)^{p-1} M_{*}^{p} M^{p} e^{-p \beta \sigma_{n}} N_{*}^{p} \sum_{k=1}^{m}\left[C_{1}+\left(d_{k}+\epsilon_{k}\right)\left(r^{*}+r\right)\right] \\
& :=\mathscr{L}_{1},
\end{aligned}
$$

where $r^{* *}=r^{*}+r+b^{p-1} \int_{0}^{b} m_{h}(s, s) \Theta_{h}(r) d s, C_{p}=(p(p-$ 1)/2) ${ }^{p / 2}$. By Lemma 6 , we have

$$
\begin{aligned}
\tilde{\rho}_{n}^{\prime}(t)= & \int_{0}^{t} C(t-s) B u_{\bar{x}}^{a}(s) d s \\
& +\lambda \int_{0}^{t} C(t-s) f(s) d w(s) \\
& +\sum_{0<t_{k}<t} A S\left(t-t_{k}\right) C\left(\sigma_{n}\right) I_{k}\left(\bar{x}_{t_{k}}, \bar{x}^{\prime}\left(t_{k}\right)\right) \\
& +\sum_{0<t_{k}<t} C\left(t-t_{k}\right) S\left(\sigma_{n}\right) J_{k}\left(\bar{x}_{t_{k}}, \bar{x}^{\prime}\left(t_{k}\right)\right),
\end{aligned}
$$

$t \in J$.

Similarly, for any $t \in J$, we have

$$
\begin{aligned}
& E\left\|\tilde{\rho}_{n}^{\prime}(t)\right\|_{H}^{p} \leq 20^{p-1} M_{*}^{p} \frac{1}{a^{p}} \\
& \cdot M^{p} e^{-p \alpha b} M_{1}^{2 p} b^{p-1} N_{*}^{2 p}\left\{4 ^ { p - 1 } \left[\left\|E \tilde{x}_{b}\right\|_{H}^{p}\right.\right. \\
& +C_{p} b^{p / 2-1} \int_{0}^{b} E\|\tilde{\phi}(\eta)\|_{H}^{p} d \eta \\
& +M^{p} e^{-p \alpha b}\left[E\|\varphi(0)\|_{H}^{p}\right.
\end{aligned}
$$

$$
\begin{aligned}
& \left.\left.+2^{p-1}\left[\|\phi\|_{H}^{p}+\|g(0, \varphi, \phi)\|_{H}^{p}\right]\right]\right]+M^{p} b^{p-1} L_{g}\left(r^{*}\right. \\
& +r+1) \int_{0}^{b} e^{-p \alpha(b-\eta)} d \eta+C_{p} M^{p} b^{p / 2-1} \Theta_{f}\left(r^{* *}\right) \\
& \cdot \int_{0}^{b} e^{-p \beta(b-\eta)} m_{f}(\eta) d \eta \\
& +m^{p-1} M^{2 p} e^{-p \gamma b} e^{-p \alpha \sigma_{n}} N_{*}^{p} \sum_{k=1}^{m}\left[C_{1}\right. \\
& \left.+\left(c_{k}+\epsilon_{k}\right)\left(r^{*}+r\right)\right] \\
& +m^{p-1} M^{2 p} e^{-p \alpha b} e^{-p \beta \sigma_{n}} N_{*}^{p} \sum_{k=1}^{m}\left[C_{1}\right. \\
& \left.\left.+\left(d_{k}+\epsilon_{k}\right)\left(r^{*}+r\right)\right]\right\}
\end{aligned}
$$$$
+4^{p-1} C_{p} M^{p} b^{p / 2-1} \Theta_{f}\left(r^{* *}\right) \int_{0}^{b} e^{-p \alpha(b-s)} m_{f}(s) d s
$$$$
+(4 m)^{p-1} M_{*}^{p} M^{p} e^{-p \alpha \sigma_{n}} N_{*}^{p} \sum_{k=1}^{m}\left[C_{1}+\left(c_{k}+\epsilon_{k}\right)\left(r^{*}\right.\right.
$$$$
+r)]+(4 m)^{p-1} M_{*}^{p} M^{p} e^{-p \beta \sigma_{n}} N_{*}^{p} \sum_{k=1}^{m}\left[C_{1}+\left(d_{k}\right.\right.
$$$$
\left.\left.+\epsilon_{k}\right)\left(r^{*}+r\right)\right]:=\mathscr{L}_{2} .
$$

Take $\mathscr{L}=\mathscr{L}_{1}+\mathscr{L}_{2}$. Then for each $\tilde{\rho}_{n} \in \Upsilon_{n} x$, we have $\|\widetilde{\rho}\|_{\mathscr{P} \mathscr{C}^{1}}^{p} \leq \mathscr{L}$. 
12

Journal of Function Spaces

(3) $\Upsilon_{n}$ is a compact multivalued map.

To this end, we decompose $\Upsilon_{n}$ by $\Upsilon_{n}=\Gamma_{n}^{1}+\Gamma_{n}^{2}$, where the map $\Gamma_{n}^{1}: B_{r}\left(0, \mathscr{B}_{b}^{0}\right) \rightarrow \mathscr{P}\left(\mathscr{B}_{b}^{0}\right)$ is defined by $\Gamma_{n}^{1} x$ and the set $\tilde{\gamma}_{n}^{1} \in \mathscr{B}_{b}^{0}$ such that

$$
\begin{aligned}
\tilde{\gamma}_{n}^{1}(t)(t)= & \int_{0}^{t} S(t-s) B u_{\bar{x}}^{a}(s) d s \\
& +\int_{0}^{t} S(t-s) f(s) d w(s),
\end{aligned}
$$

and the map $\Gamma_{n}^{2}: B_{r}\left(0, \mathscr{B}_{b}^{0}\right) \rightarrow \mathscr{P}\left(\mathscr{B}_{b}^{0}\right)$ is defined by $\Gamma_{n}^{2} x$, and the set $\tilde{\gamma}_{n}^{2} \in \mathscr{B}_{b}^{0}$ such that

$$
\begin{aligned}
\tilde{\gamma}_{n}^{2}(t)= & \sum_{0<t_{k}<t} C\left(t-t_{k}\right) C\left(\sigma_{n}\right) I_{k}\left(\bar{x}_{t_{k}}, \bar{x}^{\prime}\left(t_{k}\right)\right) \\
& +\sum_{0<t_{k}<t} S\left(t-t_{k}\right) S\left(\sigma_{n}\right) J_{k}\left(\bar{x}_{t_{k}}, \bar{x}^{\prime}\left(t_{k}\right)\right) .
\end{aligned}
$$

First, $\Gamma_{n}^{1}\left(B_{r}\left(0, \mathscr{B}_{b}^{0}\right)\right)$ is a compact multivalued map. We begin by showing that $\Gamma_{n}^{1}\left(B_{r}\left(0, \mathscr{B}_{b}^{0}\right)\right)$ is equicontinuous. Let $0<\tau_{1}<\tau_{2} \leq b$. Then, we have, for each $x \in B_{r}\left(0, \mathscr{B}_{b}^{0}\right)$,

$$
\begin{aligned}
& E\left\|\tilde{\gamma}_{n}^{1}\left(\tau_{2}\right)-\tilde{\gamma}_{n}^{1}\left(\tau_{1}\right)\right\|_{H}^{p} \leq 6^{p-1} E\left\|\int_{0}^{\tau_{1}-\varepsilon}\left[S\left(\tau_{2}-s\right)-S\left(\tau_{1}-s\right)\right] B u_{\bar{x}}(s) d s\right\|_{H}^{p}+6^{p-1} E \| \int_{\tau_{1}-\varepsilon}^{\tau_{1}}\left[S\left(\tau_{2}-s\right)-S\left(\tau_{1}-s\right)\right] \\
& \cdot B u_{\bar{x}}(s) d s\left\|_{H}^{p}+6^{p-1} E\right\| \int_{\tau_{1}}^{\tau_{2}} S\left(\tau_{2}-s\right) B u_{\bar{x}}(s) d s\left\|_{H}^{p}+6^{p-1} E\right\| \int_{0}^{\tau_{1}-\varepsilon}\left[S\left(\tau_{2}-s\right)-S\left(\tau_{1}-s\right)\right] f(s) d w(s) \|_{H}^{p} \\
& +6^{p-1} E\left\|\int_{\tau_{1}-\varepsilon}^{\tau_{1}}\left[S\left(\tau_{2}-s\right)-S\left(\tau_{1}-s\right)\right] f(s) d w(s)\right\|_{H}^{p}+6^{p-1} E\left\|\int_{\tau_{1}}^{\tau_{2}} S\left(\tau_{2}-s\right) f(s) d w(s)\right\|_{H}^{p} \\
& \leq 6^{p-1} E\left[\int_{0}^{\tau_{1}-\varepsilon}\left\|S\left(\tau_{2}-s\right)-S\left(\tau_{1}-s\right)\right\|_{H}\left\|B u_{\bar{x}}(s)\right\|_{H} d s\right]^{p}+6^{p-1} E\left[\int_{\tau_{1}-\varepsilon}^{\tau_{1}}\left\|S\left(\tau_{2}-s\right)-S\left(\tau_{1}-s\right)\right\|_{H}\left\|B u_{\bar{x}}(s)\right\|_{H} d s\right]^{p} \\
& +6^{p-1} E\left[\int_{\tau_{1}}^{\tau_{2}}\left\|S\left(\tau_{2}-s\right)\right\|_{H}\left\|B u_{\bar{x}}(s)\right\|_{H} d s\right]^{p}+6^{p-1} C_{p}\left[\int_{0}^{\tau_{1}-\varepsilon}\left[\left\|S\left(\tau_{2}-s\right)-S\left(\tau_{1}-s\right)\right\|_{H}^{p} E\|f(s)\|_{H}^{p}\right]^{2 / p} d s\right]^{p / 2} \\
& +6^{p-1} C_{p}\left[\int_{\tau_{1}-\varepsilon}^{\tau_{1}}\left[\left\|S\left(\tau_{2}-s\right)-S\left(\tau_{1}-s\right)\right\|_{H}^{p} E\|f(s)\|_{H}^{p}\right]^{2 / p} d s\right]^{p / 2}+6^{p-1} C_{p}\left[\int_{\tau_{1}}^{\tau_{2}}\left[\left\|S\left(\tau_{2}-s\right)\right\|_{H}^{p} E\|f(s)\|_{H}^{p}\right]^{2 / p} d s\right]^{p / 2} \\
& \leq 6^{p-1} b^{p} M_{1}^{p} \int_{0}^{\tau_{1}-\varepsilon}\left\|S\left(\tau_{2}-s\right)-S\left(\tau_{1}-s\right)\right\|_{H}^{p} M_{2} d s+12^{p-1} M^{p} M_{1}^{p}\left[\int_{\tau_{1}-\varepsilon}^{\tau_{1}} e^{-\beta\left(\tau_{1}-s\right)} d s\right]^{p-1} \int_{\tau_{1}-\varepsilon}^{\tau_{1}} e^{-\beta\left(\tau_{1}-s\right)} M_{2} d s \\
& +6^{p-1} M^{p} M_{1}^{p}\left[\int_{\tau_{1}}^{\tau_{2}} e^{-\beta\left(\tau_{2}-s\right)} d s\right]^{p-1} \int_{\tau_{1}}^{\tau_{2}} e^{-\beta\left(\tau_{2}-s\right)} M_{2} d s+6^{p-1} C_{p}\left[\int _ { 0 } ^ { \tau _ { 1 } - \varepsilon } \left[\left\|S\left(\tau_{2}-s\right)-S\left(\tau_{1}-s\right)\right\|_{H}^{p} m_{f}(s)\right.\right. \\
& \left.\left.\cdot \Theta_{f}\left(\left\|\bar{x}_{s}\right\|_{\mathscr{B}}^{p}+E\left\|\bar{x}^{\prime}(s)\right\|_{H}^{p}+b^{p-1} \int_{0}^{s} m_{h}(s, \tau) \Theta_{h}\left(\left\|\bar{x}_{\tau}\right\|_{\mathscr{B}}^{p}+E\left\|\bar{x}^{\prime}(\tau)\right\|_{H}^{p}\right) d \tau\right)\right]^{2 / p} d s\right]^{p / 2} \\
& +12^{p-1} C_{p} M^{p}\left[\int _ { \tau _ { 1 } - \varepsilon } ^ { \tau _ { 1 } } \left[e^{-p \beta\left(\tau_{1}-s\right)} m_{f}(s)\right.\right. \\
& \left.\left.\cdot \Theta_{f}\left(\left\|\bar{x}_{s}\right\|_{\mathscr{B}}^{p}+E\left\|\bar{x}^{\prime}(s)\right\|_{H}^{p}+b^{p-1} \int_{0}^{s} m_{h}(s, \tau) \Theta_{h}\left(\left\|\bar{x}_{\tau}\right\|_{\mathscr{B}}^{p}+E\left\|\bar{x}^{\prime}(\tau)\right\|_{H}^{p}\right) d \tau\right)\right]^{2 / p} d s\right]^{p / 2} \\
& +6^{p-1} C_{p} M^{p}\left[\int _ { \tau _ { 1 } } ^ { \tau _ { 2 } } \left[e^{-p \beta\left(\tau_{2}-s\right)} m_{f}(s)\right.\right. \\
& \left.\left.\cdot \Theta_{f}\left(\left\|\bar{x}_{s}\right\|_{\mathscr{B}}^{p}+E\left\|\bar{x}^{\prime}(s)\right\|_{H}^{p}+b^{p-1} \int_{0}^{s} m_{h}(s, \tau) \Theta_{h}\left(\left\|\bar{x}_{\tau}\right\|_{\mathscr{B}}^{p}+E\left\|\bar{x}^{\prime}(\tau)\right\|_{H}^{p}\right) d \tau\right)\right]^{2 / p} d s\right]^{p / 2} \leq 6^{p-1} b^{p} M_{1}^{p} \int_{0}^{\tau_{1}-\varepsilon} \| S\left(\tau_{2}\right. \\
& -s)-S\left(\tau_{1}-s\right) \|_{H}^{p} M_{2} d s+12^{p-1} M^{p} M_{1}^{p} \beta^{1-p} \int_{\tau_{1}-\varepsilon}^{\tau_{1}} e^{-\beta\left(\tau_{1}-s\right)} M_{2} d s+6^{p-1} M^{p} M_{1}^{p} \beta^{1-p} \int_{\tau_{1}}^{\tau_{2}} e^{-\beta\left(\tau_{2}-s\right)} M_{2} d s
\end{aligned}
$$




$$
\begin{aligned}
& +6^{p-1} C_{p} \Theta_{f}\left(r^{* *}\right)\left[\int_{0}^{\tau_{1}-\varepsilon}\left[\left\|S\left(\tau_{2}-s\right)-S\left(\tau_{1}-s\right)\right\|_{H}^{p} m_{f}(s)\right]^{2 / p} d s\right]^{p / 2}+12^{p-1} C_{p} M^{p} \Theta_{f}\left(r^{* *}\right)\left[\frac{2 \beta(p-1)}{p-2}\right]^{1-p / 2} \\
& \cdot \int_{\tau_{1}-\varepsilon}^{\tau_{1}} e^{-\beta\left(\tau_{1}-s\right)} m_{f}(s) d s+6^{p-1} C_{p} M^{p} \Theta_{f}\left(r^{* *}\right)\left[\frac{2 \beta(p-1)}{p-2}\right]^{1-p / 2} \int_{\tau_{1}}^{\tau_{2}} e^{-\beta\left(\tau_{2}-s\right)} m_{f}(s) d s,
\end{aligned}
$$

where

$$
\begin{aligned}
E & \left\|u_{\bar{x}}^{a}(s)\right\|_{H}^{p} \leq 5^{p-1} M^{p} e^{-p \beta b} \frac{1}{a^{p}} \\
& \cdot M_{1}^{p} b^{p} N_{*}^{p}\left\{4 ^ { p - 1 } \left[\left\|E \tilde{x}_{b}\right\|_{H}^{p}\right.\right. \\
& +C_{p} b^{p / 2-1} \int_{0}^{b} E\|\tilde{\phi}(\eta)\|_{H}^{p} d \eta \\
& +M^{p} e^{-p \alpha b}\left[E\|\varphi(0)\|_{H}^{p}\right. \\
& \left.\left.+2^{p-1}\left[\|\phi\|_{H}^{p}+\|g(0, \varphi, \phi)\|_{H}^{p}\right]\right]\right]+M^{p} b^{p-1} L_{g}\left(r^{*}\right. \\
& +r+1) \int_{0}^{b} e^{-p \alpha(b-\eta)} d \eta \\
& +5^{p-1} C_{p} M^{p} b^{p / 2-1} \Theta_{f}\left(r^{* *}\right) \int_{0}^{b} e^{-p \beta(b-\eta)} m_{f}(\eta) d \eta \\
& +m^{p-1} M^{2 p} e^{-p \alpha b} e^{-p \alpha \sigma_{n}} N_{*}^{p} \sum_{k=1}^{m}\left[C_{1}\right. \\
& \left.+\left(c_{k}+\epsilon_{k}\right)\left(r^{*}+r\right)\right]
\end{aligned}
$$

$$
\begin{aligned}
& +m^{p-1} M^{2 p} e^{-p \beta b} e^{-p \beta \sigma_{n}} N_{*}^{p} \sum_{k=1}^{m}\left[C_{1}\right. \\
& \left.\left.+\left(d_{k}+\epsilon_{k}\right)\left(r^{*}+r\right)\right]\right\}:=M_{2} .
\end{aligned}
$$

The fact of the compactness of $S(t)$ for $t>0$ implies the continuity in the uniform operator topology. So as $\tau_{2}-\tau_{1} \rightarrow$ 0 , with $\varepsilon$ being sufficiently small, the right-hand side of the above inequality is independent of $x \in B_{r}\left(0, \mathscr{B}_{b}^{0}\right)$ and tends to zero. The equicontinuities for the cases $\tau_{1}<\tau_{2} \leq 0$ or $\tau_{1} \leq$ $0 \leq \tau_{2} \leq b$ are very simple. Thus the set $\left\{\Gamma_{n}^{1} x: x \in B_{r}\left(0, \mathscr{B}_{b}^{0}\right)\right\}$ is equicontinuous.

We now prove that $\Gamma_{n}^{1}\left(B_{r}\left(0, \mathscr{B}_{b}^{0}\right)\right)(t)=\left\{\tilde{\gamma}_{n}^{1}(t): \widetilde{\gamma}_{n}^{1}(t) \epsilon\right.$ $\left.\Gamma_{n}^{1}\left(B_{r}\left(0, \mathscr{B}_{b}^{0}\right)\right)\right\}$ is relatively compact for every $t \in[0, b]$. Let $0<t \leq s \leq b$ be fixed and let $\varepsilon$ be a real number satisfying $0<\varepsilon<t$. For $x \in B_{r}\left(0, \mathscr{B}_{b}^{0}\right)$, we define

$$
\begin{aligned}
\tilde{\gamma}_{n, \varepsilon}^{1}(t)= & \int_{0}^{t-\varepsilon} S(t-s) B u_{\bar{x}}^{a}(s) d s \\
& +\int_{0}^{t-\varepsilon} S(t-s) f(s) d w(s),
\end{aligned}
$$

where $f \in S_{F, \bar{x}}$. Using the compactness of $S(t)$ for $t>0$, we deduce that the set $U_{\varepsilon}(t)=\left\{\tilde{\gamma}_{n, \varepsilon}^{1}(t): x \in B_{r}\left(0, \mathscr{B}_{b}^{0}\right)\right\}$ is relatively compact in $H$ for every $\varepsilon, 0<\varepsilon<t$. Moreover, for every $x \in B_{r}\left(0, \mathscr{B}_{b}^{0}\right)$ we have

$$
\begin{aligned}
E\left\|\tilde{\gamma}_{n}^{1}(t)-\tilde{\gamma}_{n, \varepsilon}^{1}(t)\right\|_{H}^{p} \leq 2^{p-1} E\left\|\int_{t-\varepsilon}^{t} S(t-s) B u_{\bar{x}}(s) d s\right\|_{H}^{p}+2^{p-1} E\left\|\int_{t-\varepsilon}^{t} S(t-s) f(s) d w(s)\right\|_{H}^{p} \\
\leq 2^{p-1} M^{p} E\left[\int_{t-\varepsilon}^{t} e^{-\beta(t-s)}\left\|B u_{\bar{x}}(s)\right\|_{H} d s\right]^{p}+2^{p-1} C_{p} M^{p}\left[\int_{t-\varepsilon}^{t}\left[e^{-p \beta(t-s)} E\|f(s)\|_{H}^{p}\right]^{2 / p} d s\right]^{p / 2} \\
\leq 2^{p-1} M^{p} M_{1}^{p}\left[\int_{t-\varepsilon}^{t} e^{-\beta(t-s)} d s\right]^{p-1} \int_{t-\varepsilon}^{t} e^{-\beta(t-s)} M_{2} d s+2^{p-1} C_{p} M^{p}\left[\int _ { t - \varepsilon } ^ { t } \left[e^{-p \beta(t-s)} m_{f}(s)\right.\right. \\
\left.\left.\cdot \Theta_{f}\left(\left\|\bar{x}_{s}\right\|_{\mathscr{B}}^{p}+E\left\|\bar{x}^{\prime}(s)\right\|_{H}^{p}+b^{p-1} \int_{0}^{s} m_{h}(s, \tau) \Theta_{h}\left(\left\|\bar{x}_{\tau}\right\|_{\mathscr{B}}^{p}+E\left\|\bar{x}^{\prime}(\tau)\right\|_{H}^{p}\right) d \tau\right)\right]^{2 / p} d s\right]^{p / 2} \\
\leq 2^{p-1} M^{p} M_{1}^{p} \beta^{1-p} \int_{t-\varepsilon}^{t} e^{-\beta(t-s)} M_{2} d s+2^{p-1} C_{p} M^{p} \Theta_{f}\left(r^{* *}\right)\left[\frac{2 \beta(p-1)}{p-2}\right]^{1-p / 2} \int_{t-\varepsilon}^{t} e^{-\beta(t-s)} m_{f}(s) d s .
\end{aligned}
$$

There are relatively compact sets arbitrarily close to the set $W(t)=\left\{\left(\Gamma_{n}^{1} x\right)(t): x \in B_{r}\left(0, \mathscr{B}_{b}^{0}\right)\right\}$, and $W(t)$ is a relatively compact in $H$. Hence, the Arzelá-Ascoli theorem shows that $\Gamma_{n}^{1}$ is a compact multivalued map. 
Secondly, $\Gamma_{n}^{2}\left(B_{r}\left(0, \mathscr{B}_{b}^{0}\right)\right)$ is a compact multivalued map. We begin by showing that $\Gamma_{n}^{2}\left(B_{r}\left(0, \mathscr{B}_{b}^{0}\right)\right)$ is equicontinuous. For each $x \in B_{r}\left(0, \mathscr{B}_{b}^{0}\right), t \in(0, b)$ is fixed, $t \in\left[t_{i}, t_{i+1}\right]$, and $\tilde{\gamma}_{n}^{2} \in \Gamma_{n}^{2} x$, such that

$$
\begin{aligned}
\tilde{\gamma}_{n}^{2}(t)= & \sum_{0<t_{k}<t} C\left(t-t_{k}\right) C\left(\sigma_{n}\right) I_{k}\left(\bar{x}_{t_{k}}, \bar{x}^{\prime}\left(t_{k}\right)\right) \\
& +\sum_{0<t_{k}<t} S\left(t-t_{k}\right) S\left(\sigma_{n}\right) J_{k}\left(\bar{x}_{t_{k}}, \bar{x}^{\prime}\left(t_{k}\right)\right) .
\end{aligned}
$$

Next, for $\tau_{1} \leq s<t \leq \tau_{2}, \varepsilon>0$, we have, using the property of compact operator,

$$
\begin{aligned}
E \| & \left.\| \widehat{\widetilde{\gamma}}_{n}^{2}\right]_{i}\left(\tau_{2}\right)-\left[\widehat{\widetilde{\gamma}}_{n}^{2}\right]_{i}\left(\tau_{1}\right) \|_{H}^{p} \leq(2 m)^{p-1} \\
& \cdot \sum_{k=1}^{m} E \|\left[C\left(\tau_{2}-t_{k}\right)-C\left(\tau_{1}-t_{k}\right)\right] C\left(\sigma_{n}\right) \\
\cdot & I_{k}\left(\bar{x}_{t_{k}}, \bar{x}^{\prime}\left(t_{k}\right)\right) \|_{H}^{p}+(2 m)^{p-1} \\
& \cdot \sum_{k=1}^{m} E \|\left[S\left(\tau_{2}-t_{k}\right)-S\left(\tau_{1}-t_{k}\right)\right] S\left(\sigma_{n}\right) \\
& \cdot J_{k}\left(\bar{x}_{t_{k}}, \bar{x}^{\prime}\left(t_{k}\right)\right) \|_{H}^{p} .
\end{aligned}
$$

As $\tau_{2}-\tau_{1} \rightarrow 0$, the right-hand side of the above inequality tends to zero independently of $x$ due to the sets $\left\{C\left(\sigma_{n}\right) I_{k}\left(\bar{x}_{t_{k}}, \bar{x}^{\prime}\left(t_{k}\right)\right): x \in B_{r}\left(0, \mathscr{B}_{b}^{0}\right)\right\},\left\{C\left(\sigma_{n}\right) J_{k}\left(\bar{x}_{t_{k}}, \bar{x}^{\prime}\left(t_{k}\right)\right):\right.$ $\left.x \in B_{r}\left(0, \mathscr{B}_{b}^{0}\right)\right\}$ which are relatively compact in $H$ and the strong continuity of $C(\cdot), S(\cdot)$. So $\left[{\widehat{\widetilde{\gamma}_{n}^{2}}}_{i}, i=1,2, \ldots, m\right.$, are equicontinuous.

Now we prove that $\left[\widehat{\widetilde{\gamma}}_{n}^{2}\right]_{i}(t), i=1,2, \ldots, m$, is relatively compact for every $t \in[0, b]$.

From the following relations

$$
\begin{aligned}
& {\left[\widehat{\widetilde{\gamma}}_{n}^{2}\right]_{i}(t)=\sum_{0<t_{k}<t} C\left(t-t_{k}\right) C\left(\sigma_{n}\right) I_{k}\left(\bar{x}_{t_{k}}, \bar{x}^{\prime}\left(t_{k}\right)\right)} \\
& +\sum_{0<t_{k}<t} S\left(t-t_{k}\right) S\left(\sigma_{n}\right) J_{k}\left(\bar{x}_{t_{k}}, \bar{x}^{\prime}\left(t_{k}\right)\right) \in(2 m)^{p-1} \\
& \quad \cdot \sum_{k=1}^{m} C\left(t-t_{k}\right) C\left(\sigma_{n}\right) I_{k}\left(B_{r^{*}}[0, \mathscr{B}], B_{r}[0, H]\right) \\
& \quad+(2 m)^{p-1} \\
& \quad \cdot \sum_{k=1}^{m} S\left(t-t_{k}\right) S\left(\sigma_{n}\right) J_{k}\left(B_{r^{*}}[0, \mathscr{B}], B_{r}[0, H]\right),
\end{aligned}
$$

we conclude that $\left.\left[\widetilde{\gamma}_{n}^{2}\left(\widehat{B_{r}\left(0, \mathscr{B}_{b}^{0}\right.}\right)\right)\right]_{i}(t), i=1,2, \ldots, m$, is relatively compact for every $t \in\left[t_{i}, t_{i+1}\right]$. By Lemma 5 , we infer that $\Gamma_{n}^{2}\left(B_{r}\left(0, \mathscr{B}_{b}^{0}\right)\right)$ is relatively compact. Moreover, using the continuity of the operators $C(t), S(t)$, for all $t \in[0, b]$, we conclude that operator $\Gamma_{n}^{2}$ is also a compact multivalued map.

(4) $\Upsilon_{n}$ has a closed graph.

Let $x^{(j)} \rightarrow x^{*}, \tilde{\rho}_{n}^{(j)} \in \Upsilon_{n} x^{(j)}, x^{(j)} \in B_{r}\left(0, \mathscr{B}_{b}^{0}\right)$, and $\tilde{\rho}_{n}^{(j)} \rightarrow$

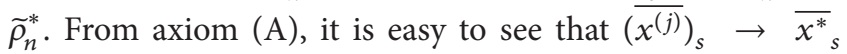

uniformly for $s \in(-\infty, b]$ as $j \rightarrow \infty$. We prove that $\tilde{\rho}_{n}^{*} \epsilon$ $\Upsilon_{n} \overline{x^{*}}$. Now $\widetilde{\rho}_{n}^{(j)} \in \Upsilon_{n} \overline{x^{(j)}}$ means that there exists $f^{(j)} \in S_{F, \overline{x^{(j)}}}$ such that, for each $t \in[0, b]$,

$$
\begin{aligned}
& \tilde{\rho}_{n}^{(j)} \\
& =\int_{0}^{t} S(t-s) B u u_{x^{(j)}}^{a}(s) d s \\
& \quad+\int_{0}^{t} S(t-s) f^{(j)}(s) d w(s) \\
& \quad+\sum_{0<t_{k}<t} C\left(t-t_{k}\right) C\left(\sigma_{n}\right) I_{k}\left(\left(\overline{x^{(j)}}\right)_{t_{k}},\left(\overline{x^{(j)}}\right)^{\prime}\left(t_{k}\right)\right) \\
& \quad+\sum_{0<t_{k}<t} S\left(\sigma_{n}\right) S\left(t-t_{k}\right) J_{k}\left(\left(\overline{x^{(j)}}\right)_{t_{k}},\left(\overline{x^{(j)}}\right)^{\prime}\left(t_{k}\right)\right), \\
& \quad t \in J,
\end{aligned}
$$

where

$$
\begin{aligned}
& u \frac{a}{x^{(j)}}(t)=B^{*} S^{*}(b-t)\left(a I+\Gamma_{0}^{b}\right)^{-1}\left[E \widetilde{x}_{b}\right. \\
& +\int_{0}^{b} \widetilde{\phi}(s) d w(s)-C(b) \varphi(0)-S(b) \\
& \cdot[\phi-g(0, \varphi, \phi)]]-B^{*} S^{*}(b-t) \int_{0}^{b}\left(a I+\Gamma_{s}^{b}\right)^{-1} \\
& \left.\cdot C(b-s) g\left(s, \overline{x^{(j)}}\right)_{s},\left(\overline{x^{(j)}}\right)^{\prime}(s)\right) d s \\
& -B^{*} S^{*}(b-t) \int_{0}^{b}\left(a I+\Gamma_{s}^{b}\right)^{-1} S(b-s) \\
& \cdot f^{(j)}(s) d w(s)-B^{*} S^{*}(b-t)\left(a I+\Gamma_{s}^{b}\right)^{-1} \\
& \cdot \sum_{k=1}^{m} C\left(b-t_{k}\right) C\left(\sigma_{n}\right) I_{k}\left(\left(\overline{x^{(j)}}\right)_{t_{k}},\left(\overline{x^{(j)}}\right)^{\prime}\left(t_{k}\right)\right) \\
& -B^{*} \delta_{\alpha}^{*}(b-t)\left(a I+\Gamma_{s}^{b}\right)^{-1} \sum_{k=1}^{m} S\left(b-t_{k}\right) S\left(\sigma_{n}\right) \\
& \cdot J_{k}\left(\left(\overline{x^{(j)}}\right)_{t_{k}},\left(\overline{x^{(j)}}\right)^{\prime}\left(t_{k}\right)\right) .
\end{aligned}
$$

We must prove that there exists $f_{*} \in S_{F, \overline{x^{*}}}$ such that, for each $t \in[0, b]$,

$$
\begin{aligned}
\tilde{\rho}_{n}^{*}(t) & \\
= & \int_{0}^{t} S(t-s) B u_{\overline{x^{*}}}(s) d s \\
& +\int_{0}^{t} S(t-s) f_{*}(s) d w(s) \\
& +\sum_{0<t_{k}<t} C\left(t-t_{k}\right) C\left(\sigma_{n}\right) I_{k}\left(\left(\overline{x^{*}}\right)_{t_{k}},\left(\overline{x^{*}}\right)^{\prime}\left(t_{k}\right)\right)
\end{aligned}
$$




$$
\begin{array}{r}
+\sum_{0<t_{k}<t} S\left(t-t_{k}\right) S\left(\sigma_{n}\right) J_{k}\left(\left(\overline{x^{*}}\right)_{t_{k}},\left(\overline{x^{*}}\right)^{\prime}\left(t_{k}\right)\right), \\
t \in J,
\end{array}
$$

where

$$
\begin{aligned}
& u \frac{a}{\bar{x}^{*}}(t)=B^{*} S^{*}(b-t)\left(a I+\Gamma_{0}^{b}\right)^{-1}\left[E \tilde{x}_{b}\right. \\
& +\int_{0}^{b} \tilde{\phi}(s) d w(s)-C(b) \varphi(0)-S(b) \\
& \cdot[\phi-g(0, \varphi, \phi)]]-B^{*} S^{*}(b-t) \int_{0}^{b}\left(a I+\Gamma_{s}^{b}\right)^{-1} \\
& \cdot C(b-s) g\left(s,\left(\overline{x^{*}}\right)_{s},\left(\overline{x^{*}}\right)^{\prime}(s)\right) d s-B^{*} S^{*}(b \\
& -t) \int_{0}^{b}\left(a I+\Gamma_{s}^{b}\right)^{-1} S(b-s) f^{*}(s) d w(s) \\
& -B^{*} S^{*}(b-t)\left(a I+\Gamma_{s}^{b}\right)^{-1} \sum_{k=1}^{m} C\left(b-t_{k}\right) C\left(\sigma_{n}\right) \\
& \cdot I_{k}\left(\left(\overline{x^{*}}\right)_{t_{k}},\left(\overline{x^{*}}\right)^{\prime}\left(t_{k}\right)\right)-B^{*} \delta_{\alpha}^{*}(b-t)(a I \\
& \left.+\Gamma_{s}^{b}\right)^{-1} \sum_{k=1}^{m} S\left(b-t_{k}\right) S\left(\sigma_{n}\right) J_{k}\left(\left(\overline{x^{*}}\right)_{t_{k}},\left(\overline{x^{*}}\right)^{\prime}\left(t_{k}\right)\right) .
\end{aligned}
$$

Since $g, I_{k}, J_{k}, k=1,2, \ldots, m$, are continuous, we obtain that

$$
\begin{aligned}
\| \widetilde{\rho}_{n}^{(j)}-\int_{0}^{t} S(t-s) B\left\{B^{*} S^{*}(b-s)\left(a I+\Gamma_{0}^{b}\right)^{-1}\right. \\
\cdot\left[E \widetilde{x}_{b}+\int_{0}^{b} \widetilde{\phi}(\eta) d w(\eta)-C(b) \varphi(0)\right. \\
-S(b)[\phi-g(0, \varphi, \phi)]]-B^{*} S^{*}(b-s) \\
\cdot \int_{0}^{b}\left(a I+\Gamma_{\eta}^{b}\right)^{-1} C(b-\eta) g \\
\cdot\left(\eta,\left(\overline{x^{(j)}}\right)_{\eta},\left(\overline{x^{(j)}}\right)^{\prime}(\eta)\right) d \eta \\
-B^{*} S^{*}(b-s)\left(a I+\Gamma_{\eta}^{b}\right)^{-1} \sum_{k=1}^{m} C\left(b-t_{k}\right) C\left(\sigma_{n}\right) I_{k} \\
\cdot\left(\left(\overline{x^{(j)}}\right)_{t_{k}},\left(\overline{x^{(j)}}\right)^{\prime}\left(t_{k}\right)\right)-B^{*} \mathcal{S}_{\alpha}^{*}(b-s)\left(a I+\Gamma_{\eta}^{b}\right)^{-1} \\
\left.\quad \cdot \sum_{k=1}^{m} S\left(b-t_{k}\right) S\left(\sigma_{n}\right) J_{k}\left(\left(\overline{x^{(j)}}\right)_{t_{k}},\left(\overline{x^{(j)}}\right)^{\prime}\left(t_{k}\right)\right)\right\} d s \\
\quad-\sum_{0<t_{k}<t} C\left(t-t_{k}\right) I_{k}\left(\left(\overline{x^{(j)}}\right)_{t_{k}},\left(\overline{x^{(j)}}\right)^{\prime}\left(t_{k}\right)\right)
\end{aligned}
$$

$$
\begin{aligned}
& -\sum_{0<t_{k}<t} S\left(t-t_{k}\right) J_{k}\left(\left(\overline{x^{(j)}}\right)_{t_{k}},\left(\overline{x^{(j)}}\right)^{\prime}\left(t_{k}\right)\right) \\
& -\left(\tilde{\rho}_{n}^{*}(t)-\int_{0}^{t} S(t-s) B\right.
\end{aligned}
$$$$
\left\{B ^ { * } S ^ { * } ( b - s ) ( a I + \Gamma _ { 0 } ^ { b } ) ^ { - 1 } \left[E \widetilde{x}_{b}+\int_{0}^{b} \tilde{\phi}(\eta) d w(\eta)\right.\right.
$$$$
-C(b) \varphi(0)-S(b)[\phi-g(0, \varphi, \phi)]]
$$$$
-B^{*} S^{*}(b-s) \int_{0}^{b}\left(a I+\Gamma_{\eta}^{b}\right)^{-1} C(b-\eta) g
$$$$
\cdot\left(\eta,\left(\overline{x^{*}}\right)_{\eta},\left(\overline{x^{*}}\right)^{\prime}(\eta)\right) d \eta-B^{*} S^{*}(b-s)\left(a I+\Gamma_{\eta}^{b}\right)^{-1}
$$$$
\cdot \sum_{k=1}^{m} C\left(b-t_{k}\right) C\left(\sigma_{n}\right) I_{k}\left(\left(\overline{x^{*}}\right)_{t_{k}},\left(\overline{x^{*}}\right)^{\prime}\left(t_{k}\right)\right)
$$$$
-B^{*} \mathcal{S}_{\alpha}^{*}(b-s)\left(a I+\Gamma_{\eta}^{b}\right)^{-1} \sum_{k=1}^{m} S\left(b-t_{k}\right) S\left(\sigma_{n}\right) J_{k}
$$$$
\left.\cdot\left(\left(\overline{x^{*}}\right)_{t_{k}},\left(\overline{x^{*}}\right)^{\prime}\left(t_{k}\right)\right)\right\} d s-\sum_{0<t_{k}<t} C\left(t-t_{k}\right) I_{k}
$$$$
\cdot\left(\left(\overline{x^{*}}\right)_{t_{k}},\left(\overline{x^{*}}\right)^{\prime}\left(t_{k}\right)\right)-\sum_{0<t_{k}<t} S\left(t-t_{k}\right) J_{k}
$$

$$
\left.\cdot\left(\left(\overline{x^{*}}\right)_{t_{k}},\left(\overline{x^{*}}\right)^{\prime}\left(t_{k}\right)\right)\right) \|_{H}^{p} \longrightarrow 0
$$

Consider the linear continuous operator $\Gamma: L^{p}(J, H) \rightarrow$ $C(J, H)$,

$$
\begin{gathered}
\Gamma(f)(t)=\int_{0}^{t} S(t-s) f(s) d w(s)+\int_{0}^{t} S(t-s) \\
\cdot\left[B B^{*} S^{*}(b-s)\right. \\
\left.\cdot\left(\int_{0}^{b}\left(a I+\Gamma_{\eta}^{b}\right)^{-1} S(b-\eta) f(\eta) d \eta\right)\right] d s .
\end{gathered}
$$

From Lemma 14, it follows that $\Gamma \circ S_{F}$ is a closed graph operator. Also, from the definition of $\Gamma$, we have that, for every $t \in[0, b]$,

$$
\begin{gathered}
\widetilde{\rho}_{n}^{(j)}-\int_{0}^{t} S(t-s) B\left\{B ^ { * } S ^ { * } ( b - s ) ( a I + \Gamma _ { 0 } ^ { b } ) ^ { - 1 } \left[E \widetilde{x}_{b}\right.\right. \\
+\int_{0}^{b} \tilde{\phi}(\eta) d w(\eta)-C(b) \varphi(0)-S(b)
\end{gathered}
$$




$$
\begin{aligned}
& \cdot[\phi-g(0, \varphi, \phi)]]-B^{*} S^{*}(b-s) \int_{0}^{b}\left(a I+\Gamma_{\eta}^{b}\right)^{-1} \\
& \cdot C(b-\eta) g\left(\eta,\left(\overline{x^{(j)}}\right)_{\eta},\left(\overline{x^{(j)}}\right)^{\prime}(\eta)\right) d \eta \\
& -B^{*} S^{*}(b-s)\left(a I+\Gamma_{\eta}^{b}\right)^{-1} \sum_{k=1}^{m} C\left(b-t_{k}\right) C\left(\sigma_{n}\right) \\
& \cdot I_{k}\left(\left(\overline{x^{(j)}}\right)_{t_{k}},\left(\overline{x^{(j)}}\right)^{\prime}\left(t_{k}\right)\right)-B^{*} S_{\alpha}^{*}(b-s)(a I \\
& \left.+\Gamma_{\eta}^{b}\right)^{-1} \sum_{k=1}^{m} S\left(b-t_{k}\right) S\left(\sigma_{n}\right) \\
& \left.\cdot J_{k}\left(\left(\overline{x^{(j)}}\right)_{t_{k}},\left(\overline{x^{(j)}}\right)^{\prime}\left(t_{k}\right)\right)\right\} d s-\sum_{0<t_{k}<t} C(t \\
& \left.-t_{k}\right) I_{k}\left(\left(\overline{x^{(j)}}\right)_{t_{k}},\left(\overline{x^{(j)}}\right)^{\prime}\left(t_{k}\right)\right)-\sum_{0<t_{k}<t} S\left(t-t_{k}\right) \\
& \cdot J_{k}\left(\left(\overline{x^{(j)}}\right)_{t_{k}},\left(\overline{x^{(j)}}\right)^{\prime}\left(t_{k}\right)\right) \in \Gamma\left(S_{F, \overline{x^{(j)}}}\right) .
\end{aligned}
$$

Since $\overline{x^{(j)}} \rightarrow \overline{x^{*}}$, for some $f^{*} \in S_{F, x^{*}}$ it follows that, for every $t \in[0, b]$, we have

$$
\begin{aligned}
& \tilde{\rho}_{n}^{*}(t)-\int_{0}^{t} S(t-s) B\left\{B ^ { * } S ^ { * } ( b - s ) ( a I + \Gamma _ { 0 } ^ { b } ) ^ { - 1 } \left[E \tilde{x}_{b}\right.\right. \\
& +\int_{0}^{b} \tilde{\phi}(\eta) d w(\eta)-C(b) \varphi(0)-S(b) \\
& \cdot[\phi-g(0, \varphi, \phi)]]-B^{*} S^{*}(b-s) \int_{0}^{b}\left(a I+\Gamma_{s}^{b}\right)^{-1} \\
& \cdot C(b-\eta) g\left(\eta,\left(\overline{x^{*}}\right)_{\eta},\left(\overline{x^{*}}\right)^{\prime}(\eta)\right) d \eta-B^{*} S^{*}(b \\
& -s)\left(a I+\Gamma_{\eta}^{b}\right)^{-1} \sum_{k=1}^{m} C\left(b-t_{k}\right) C\left(\sigma_{n}\right) \\
& \cdot I_{k}\left(\left(\overline{x^{*}}\right)_{t_{k}},\left(\overline{x^{*}}\right)^{\prime}\left(t_{k}\right)\right)-B^{*} S_{\alpha}^{*}(b-s)(a I \\
& \left.+\Gamma_{s}^{b}\right)^{-1} \sum_{k=1}^{m} S\left(b-t_{k}\right) S\left(\sigma_{n}\right) \\
& \left.\cdot J_{k}\left(\left(\overline{x^{*}}\right)_{t_{k}},\left(\overline{x^{*}}\right)^{\prime}\left(t_{k}\right)\right)\right\} d s-\sum_{0<t_{k}<t} C\left(t-t_{k}\right) \\
& \cdot I_{k}\left(\left(\overline{x^{*}}\right)_{t_{k}},\left(\overline{x^{*}}\right)^{\prime}\left(t_{k}\right)\right)-\sum_{0<t_{k}<t} S\left(t-t_{k}\right)
\end{aligned}
$$

$$
\begin{aligned}
& \cdot J_{k}\left(\left(\overline{x^{*}}\right)_{t_{k}},\left(\overline{x^{*}}\right)^{\prime}\left(t_{k}\right)\right)=\int_{0}^{t} S(t-s) f^{*}(s) d w(s) \\
& +\int_{0}^{t} S(t-s)\left[B B^{*} S^{*}(b-s)\right. \\
& \left.\cdot\left(\int_{0}^{b}\left(a I+\Gamma_{\eta}^{b}\right)^{-1} S(b-\eta) f^{*}(\eta) d \eta\right)\right] d s .
\end{aligned}
$$

Therefore, $\Upsilon_{n}$ is a completely continuous multivalued map, u.s.c. with convex closed, compact values.

Step 3. We will show that the set $G=\left\{x \in \mathscr{B}_{b}^{0}: x \in \lambda \Lambda_{n} x+\right.$ $\lambda \Upsilon_{n} x$ for some $\left.\lambda \in(0,1)\right\}$ is bounded on $J$.

Let $x \in \mathscr{B}_{b}^{0}$, and then there exists $f \in S_{F, \bar{x}}$ such that we have

$$
\begin{aligned}
x(t)= & \lambda \int_{0}^{t} C(t-s) g\left(s, \bar{x}_{s}, \bar{x}^{\prime}(s)\right) d s \\
& +\lambda \int_{0}^{t} S(t-s) B u_{\bar{x}}^{a}(s) d s \\
& +\lambda \int_{0}^{t} S(t-s) f(s) d w(s) \\
& +\lambda \sum_{0<t_{k}<t} C\left(t-t_{k}\right) C\left(\sigma_{n}\right) I_{k}\left(\bar{x}_{t_{k}}, \bar{x}^{\prime}\left(t_{k}\right)\right) \\
& +\lambda \sum_{0<t_{k}<t} S\left(t-t_{k}\right) S\left(\sigma_{n}\right) J_{k}\left(\bar{x}_{t_{k}}, \bar{x}^{\prime}\left(t_{k}\right)\right),
\end{aligned}
$$

$t \in J$.

It also follows from Lemma 6 that

$$
\begin{aligned}
x^{\prime}(t)= & \lambda g\left(t, \bar{x}_{t}, \bar{x}^{\prime}(t)\right) \\
& +\lambda \int_{0}^{t} A S(t-s) g\left(s, \bar{x}_{s}, \bar{x}^{\prime}(s)\right) d s \\
& +\lambda \int_{0}^{t} C(t-s) B u_{\bar{x}}^{a}(s) d s \\
& +\lambda \int_{0}^{t} C(t-s) f(s) d w(s) \\
& +\lambda \sum_{0<t_{k}<t} A S\left(t-t_{k}\right) C\left(\sigma_{n}\right) I_{k}\left(\bar{x}_{t_{k}}, \bar{x}^{\prime}\left(t_{k}\right)\right) \\
& +\lambda \sum_{0<t_{k}<t} C\left(t-t_{k}\right) S\left(\sigma_{n}\right) J_{k}\left(\bar{x}_{t_{k}}, \bar{x}^{\prime}\left(t_{k}\right)\right), \\
& \quad t \in J .
\end{aligned}
$$

This implies by (H1)-(H5) and (41) that for each $t \in J$ we have 


$$
\begin{aligned}
E & \|x(t)\|_{H}^{p} \leq 5^{p-1} E\left\|\int_{0}^{t} C(t-s) g\left(s, \bar{x}_{s}, \bar{x}^{\prime}(s)\right) d s\right\|_{H}^{p}+5^{p-1} E\left\|\int_{0}^{t} S(t-s) B u_{\bar{x}}^{a}(s) d s\right\|_{H}^{p}+5^{p-1} E \| \int_{0}^{t} S(t-s) \\
\cdot & f(s) d w(s)\left\|_{H}^{p}+5^{p-1} E\right\| \sum_{0<t_{k}<t} C\left(t-t_{k}\right) C\left(\sigma_{n}\right) I_{k}\left(\bar{x}_{t_{k}}, \bar{x}^{\prime}\left(t_{k}\right)\right)\left\|_{H}^{p}+5^{p-1} E\right\| \sum_{0<t_{k}<t} s\left(t-t_{k}\right) S\left(\sigma_{n}\right) \\
\cdot & J_{k}\left(\bar{x}_{t_{k}}, \bar{x}^{\prime}\left(t_{k}\right)\right) \|_{H}^{p} \leq 5^{p-1} M^{p} b^{p-1} L_{g} \int_{0}^{t} e^{-p \alpha(t-s)}\left[\left\|\bar{x}_{s}\right\|_{\mathscr{B}}^{p}+E\left\|\bar{x}^{\prime}(s)\right\|_{H}^{p}+1\right] d s+20^{p-1} M^{p} e^{-p \beta t} \frac{1}{a^{p}} \\
& \cdot M^{p} e^{-p \beta b} M_{1}^{2 p} b^{p-1} \int_{0}^{t} e^{2 p \beta s}\left\{4 ^ { p - 1 } \left[\left\|E \tilde{x}_{b}\right\|_{H}^{p}+C_{p} b^{p / 2-1} \int_{0}^{b} E\|\widetilde{\phi}(\eta)\|_{H}^{p} d \eta\right.\right. \\
& \left.+M^{p} e^{-p \alpha b}\left[E\|\varphi(0)\|_{H}^{p}+2^{p-1}\left[\|\phi\|_{H}^{p}+\|g(0, \varphi, \phi)\|_{H}^{p}\right]\right]\right]+M^{p} b^{p-1} L_{g} \int_{0}^{b} e^{-p \alpha(b-\eta)}\left[\left\|\bar{x}_{\eta}\right\|_{\mathscr{B}}^{p}+E\left\|\bar{x}^{\prime}(\eta)\right\|_{H}^{p}+1\right] d \eta \\
& +C_{p} M^{p} b^{p / 2-1} \int_{0}^{b} e^{-p \beta(b-\eta)} m_{f}(\eta) \Theta_{f}\left(\left\|\bar{x}_{\eta}\right\|_{\mathscr{B}}^{p}+E\left\|\bar{x}^{\prime}(\eta)\right\|_{H}^{p}+b^{p-1} \int_{0}^{\eta} m_{h}(\eta, v) \Theta_{h}\left(\left\|\bar{x}_{v}\right\|_{\mathscr{B}}^{p}+E\left\|\bar{x}^{\prime}(v)\right\|_{H}^{p}\right) d \nu\right) d \eta \\
& +m^{p-1} M^{2 p} \sum_{k=1}^{m} e^{-p \alpha\left(b-t_{k}\right)} e^{-p \alpha \sigma_{n}}\left[C_{1}+\left(c_{k}+\epsilon_{k}\right)\left(\left\|\bar{x}_{t_{k}}\right\|_{\mathscr{B}}^{p}+E\left\|\bar{x}^{\prime}\left(t_{k}\right)\right\|_{H}^{p}\right)\right] \\
& \left.+m^{p-1} M^{2 p} \sum_{k=1}^{m} e^{-p \beta\left(b-t_{k}\right)} e^{-p \beta \sigma_{n}}\left[C_{1}+\left(d_{k}+\epsilon_{k}\right)\left(\left\|\bar{x}_{t_{k}}\right\|_{\mathscr{B}}^{p}+E\left\|\bar{x}^{\prime}\left(t_{k}\right)\right\|_{H}^{p}\right)\right]\right\} d s+5^{p-1} C_{p} M^{p} b^{p / 2-1} \int_{0}^{t} e^{-p \beta(t-s)} m_{f}(s) \\
& \cdot \Theta_{f}\left(\left\|\bar{x}_{s}\right\|_{\mathscr{B}}^{p}+E\left\|\bar{x}^{\prime}(s)\right\|_{H}^{p}+b^{p-1} \int_{0}^{s} m_{h}(s, \tau) \Theta_{h}\left(\left\|\bar{x}_{\tau}\right\|_{\mathscr{B}}^{p}+E\left\|\bar{x}^{\prime}(\tau)\right\|_{H}^{p}\right) d \tau\right) d s+(5 m)^{p-1} \\
& \cdot M^{2 p} \sum_{k=1}^{m} e^{-p \alpha\left(t-t_{k}\right)} e^{-p \alpha \sigma_{n}}\left[C_{1}+\left(c_{k}+\epsilon_{k}\right)\left(\left\|\bar{x}_{t_{k}}\right\|_{\mathscr{B}}^{p}+E\left\|\bar{x}_{B}^{p}+E\right\| \bar{x}^{\prime}\left(t_{k}\right) \|_{H}^{p}\right)\right] . \\
&
\end{aligned}
$$

$$
\begin{aligned}
& E\left\|x^{\prime}(t)\right\|_{H}^{p} \leq 6^{p-1} L_{g}\left[\left\|\bar{x}_{t}\right\|_{\mathscr{B}}^{p}+E\left\|\bar{x}^{\prime}(t)\right\|_{H}^{p}+1\right]+6^{p-1} M^{p} b^{p-1} L_{g} \int_{0}^{t} e^{-p \gamma(t-s)}\left[\left\|\bar{x}_{s}\right\|_{\mathscr{B}}^{p}+E\left\|\bar{x}^{\prime}(s)\right\|_{H}^{p}+1\right] d s \\
& +20^{p-1} M^{p} e^{-p \alpha t} \frac{1}{a^{p}} M^{p} e^{-p \alpha b} M_{1}^{2 p} b^{p-1} \int_{0}^{t} e^{2 p \alpha s}\left\{4 ^ { p - 1 } \left[\left\|E \tilde{x}_{b}\right\|_{H}^{p}+C_{p} b^{p / 2-1} \int_{0}^{b} E\|\widetilde{\phi}(\eta)\|_{H}^{p} d \eta\right.\right. \\
& \left.+M^{p} e^{-p \alpha b}\left[E\|\varphi(0)\|_{H}^{p}+2^{p-1}\left[\|\phi\|_{H}^{p}+\|g(0, \varphi, \phi)\|_{H}^{p}\right]\right]\right]+M^{p} b^{p-1} L_{g} \int_{0}^{b} e^{-p \alpha(b-\eta)}\left[\left\|\bar{x}_{\eta}\right\|_{\mathscr{B}}^{p}+E\left\|\bar{x}^{\prime}(\eta)\right\|_{H}^{p}+1\right] d \eta \\
& +C_{p} M^{p} b^{p / 2-1} \int_{0}^{b} e^{-p \beta(b-\eta)} m_{f}(\eta) \Theta_{f}\left(\left\|\bar{x}_{\eta}\right\|_{\mathscr{B}}^{p}+E\left\|\bar{x}^{\prime}(\eta)\right\|_{H}^{p}+b^{p-1} \int_{0}^{\eta} m_{h}(\eta, v) \Theta_{h}\left(\left\|\bar{x}_{v}\right\|_{\mathscr{B}}^{p}+E\left\|\bar{x}^{\prime}(v)\right\|_{H}^{p}\right) d \nu\right) d \eta \\
& +m^{p-1} M^{2 p} \sum_{k=1}^{m} e^{-p \alpha\left(b-t_{k}\right)} e^{-p \alpha \sigma_{n}}\left[C_{1}+\left(c_{k}+\epsilon_{k}\right)\left(\left\|\bar{x}_{t_{k}}\right\|_{\mathscr{B}}^{p}+E\left\|\bar{x}^{\prime}\left(t_{k}\right)\right\|_{H}^{p}\right)\right] \\
& \left.+m^{p-1} M^{2 p} \sum_{k=1}^{m} e^{-p \beta\left(b-t_{k}\right)} e^{-p \beta \sigma_{n}}\left[C_{1}+\left(d_{k}+\epsilon_{k}\right)\left(\left\|\bar{x}_{t_{k}}\right\|_{\mathscr{B}}^{p}+E\left\|\bar{x}^{\prime}\left(t_{k}\right)\right\|_{H}^{p}\right)\right]\right\} d s+5^{p-1} C_{p} M^{p} b^{p / 2-1} \int_{0}^{t} e^{-p \alpha(t-s)} m_{f}(s)
\end{aligned}
$$




$$
\begin{aligned}
& \cdot \Theta_{f}\left(\left\|\bar{x}_{s}\right\|_{\mathscr{B}}^{p}+E\left\|\bar{x}^{\prime}(s)\right\|_{H}^{p}+b^{p-1} \int_{0}^{s} m_{h}(s, \tau) \Theta_{h}\left(\left\|\bar{x}_{\tau}\right\|_{\mathscr{B}}^{p}+E\left\|\bar{x}^{\prime}(\tau)\right\|_{H}^{p}\right) d \tau\right) d s+(5 m)^{p-1} M^{2 p} \sum_{k=1}^{m} e^{-\gamma p\left(t-t_{k}\right)}\left[C_{1}\right. \\
& \left.+\left(c_{k}+\epsilon_{k}\right)\left(\left\|\bar{x}_{t_{k}}\right\|_{B}^{p}+E\left\|\bar{x}^{\prime}\left(t_{k}\right)\right\|_{H}^{p}\right)\right]+(5 m)^{p-1} M^{2 p} \sum_{k=1}^{m} e^{-\alpha p\left(t-t_{k}\right)}\left[C_{1}+\left(d_{k}+\epsilon_{k}\right)\left(\left\|\bar{x}_{t_{k}}\right\|_{B}^{p}+E\left\|\bar{x}^{\prime}\left(t_{k}\right)\right\|_{H}^{p}\right)\right] .
\end{aligned}
$$

By Lemma 11, it follows that

$$
\begin{aligned}
\sup & \left\{\left\|\bar{x}_{s}\right\|_{\mathscr{B}}^{p}: 0 \leq s \leq t\right\} \\
& \leq 2^{p-1}\left(M_{b} E\|\varphi\|_{\mathscr{B}}\right)^{p} \\
& +2^{p-1} K_{b}^{p} \sup \left\{E\|x(s)\|_{H}^{p}: 0 \leq s \leq t\right\} .
\end{aligned}
$$

Consider the function defined by

$$
\begin{aligned}
\mu(t)= & 2^{p-1}\left(M_{b} E\|\varphi\|_{\mathscr{B}}\right)^{p} \\
& +2^{p-1} K_{b}^{p} \sup \left\{E\|x(s)\|_{H}^{p}: 0 \leq s \leq t\right\} \\
& +2^{p-1} \sup \left\{E\left\|\bar{x}^{\prime}(s)\right\|_{H}^{p}: 0 \leq s \leq t\right\},
\end{aligned}
$$

$$
0 \leq t \leq b
$$

where

$$
\begin{aligned}
& \left\|\bar{x}_{s}\right\|_{\mathscr{B}}^{p}+E\left\|\bar{x}^{\prime}(s)\right\|_{H}^{p} \\
& \leq \sup \left\{\left\|\bar{x}_{s}\right\|_{\mathscr{B}}^{p}: 0 \leq s \leq t\right\} \\
& \quad+\sup \left\{E\left\|\bar{x}^{\prime}(s)\right\|_{H}^{p}: 0 \leq s \leq t\right\} .
\end{aligned}
$$

For each $t \in[0, b]$, we have

$$
\begin{aligned}
E & \|x(t)\|_{H}^{p} \leq 5^{p-1} M^{p} b^{p-1} L_{g} e^{-p \alpha t} \int_{0}^{t} e^{p \alpha s} \mu(s) d s \\
& +20^{p-1} M^{p} e^{-p \beta t} \frac{1}{a^{p}} \\
& +M^{p} e^{-p \beta b} M_{1}^{2 p} b^{p-1} \int_{0}^{t} e^{2 p \beta s}\left\{4 ^ { p - 1 } \left[\left\|E \tilde{x}_{b}\right\|_{H}^{p}\right.\right. \\
& +C_{p} b^{p / 2-1} \int_{0}^{b} E\|\widetilde{\phi}(\eta)\|_{H}^{p} d \eta \\
& \left.+M^{p} e^{-p \alpha b}\left[E\|\varphi(0)\|_{H}^{p}+2^{p-1}\left[\|\phi\|_{H}^{p}+\|g(0, \varphi, \phi)\|_{H}^{p}\right]\right]\right] \\
& +M^{p} b^{p-1} L_{g} \int_{0}^{b} e^{-p \alpha(b-\eta)}[\mu(\eta)+1] d \eta
\end{aligned}
$$

$$
\begin{aligned}
& +C_{p} M^{p} b^{p / 2-1} \int_{0}^{b} e^{-p \beta(b-\eta)} m_{f}(\eta) \\
& \cdot \Theta_{f}\left(\mu(\eta)+b^{p-1} \int_{0}^{\eta} m_{h}(\eta, \nu) \Theta_{h}(\mu(\nu)) d \nu\right) d \eta \\
& +m^{p-1} M^{2 p} \sum_{k=1}^{m} e^{-p \alpha\left(b-t_{k}\right)} e^{-p \alpha \sigma_{n}}\left[C_{1}+\left(c_{k}+\epsilon_{k}\right) \mu(\eta)\right] \\
& \left.+m^{p-1} M^{2 p} \sum_{k=1}^{m} e^{-p \beta\left(b-t_{k}\right)} e^{-p \alpha \sigma_{n}}\left[C_{1}+\left(d_{k}+\epsilon_{k}\right) \mu(\eta)\right]\right\} d s \\
& +5^{p-1} C_{p} M^{p} b^{p / 2-1} \int_{0}^{t} e^{-p \alpha(t-s)} m_{f}(s) \Theta_{f}(\mu(s) \\
& \left.+b^{p-1} \int_{0}^{s} m_{h}(s, \tau) \Theta_{h}(\mu(\tau)) d \tau\right) d s+(5 m)^{p-1} \\
& \cdot M^{2 p} \sum_{k=1}^{m} e^{-p \alpha\left(t-t_{k}\right)} e^{-p \alpha \sigma_{n}}\left[C_{1}+\left(c_{k}+\epsilon_{k}\right) \mu(s)\right]+(5 m)^{p-1}
\end{aligned}
$$$$
\cdot M^{2 p} \sum_{k=1}^{m} e^{-p \beta\left(t-t_{k}\right)} e^{-p \alpha \sigma_{n}}\left[C_{1}+\left(d_{k}+\epsilon_{k}\right) \mu(s)\right],
$$$$
E\left\|x^{\prime}(t)\right\|_{H}^{p} \leq 6^{p-1} L_{g}[\mu(t)+1]
$$$$
+6^{p-1} M^{p} b^{p-1} L_{g} e^{-p \gamma t} \int_{0}^{t} e^{p \gamma s} \mu(s) d s+24^{p-1} M^{p} e^{-p \alpha t} \frac{1}{a^{p}}
$$$$
\cdot M^{p} e^{-p \alpha b} M_{1}^{2 p} b^{p-1} \int_{0}^{t} e^{2 p \alpha s}\left\{4 ^ { p - 1 } \left[\left\|E \tilde{x}_{b}\right\|_{H}^{p}\right.\right.
$$$$
+C_{p} b^{p / 2-1} \int_{0}^{b} E\|\widetilde{\phi}(\eta)\|_{H}^{p} d \eta
$$$$
\left.+M^{p} e^{-p \alpha b}\left[E\|\varphi(0)\|_{H}^{p}+2^{p-1}\left[\|\phi\|_{H}^{p}+\|g(0, \varphi, \phi)\|_{H}^{p}\right]\right]\right]
$$$$
+M^{p} b^{p-1} L_{g} \int_{0}^{b} e^{-p \alpha(b-\eta)}[\mu(\eta)+1] d \eta
$$$$
+C_{p} M^{p} b^{p / 2-1} \int_{0}^{b} e^{-p \beta(b-\eta)} m_{f}(\eta)
$$$$
\cdot \Theta_{f}\left(\mu(\eta)+b^{p-1} \int_{0}^{\eta} m_{h}(\eta, \nu) \Theta_{h}(\mu(\nu)) d \nu\right) d \eta
$$$$
+m^{p-1} M^{2 p} \sum_{k=1}^{m} e^{-p \alpha\left(b-t_{k}\right)} e^{-p \alpha \sigma_{n}}\left[C_{1}+\left(c_{k}+\epsilon_{k}\right)(\mu(\eta))\right]
$$ 


$$
\begin{aligned}
& \left.+m^{p-1} M^{2 p} \sum_{k=1}^{m} e^{-p \beta\left(b-t_{k}\right)} e^{-p \alpha \sigma_{n}}\left[C_{1}+\left(d_{k}+\epsilon_{k}\right)(\mu(\eta))\right]\right\} d s \\
& +6^{p-1} C_{p} M^{p} b^{p / 2-1} \int_{0}^{t} e^{-p \alpha(t-s)} m_{f}(s) \Theta_{f}(\mu(s) \\
& \left.+b^{p-1} \int_{0}^{s} m_{h}(s, \tau) \Theta_{h}(\mu(\tau)) d \tau\right) d s+(6 m)^{p-1} \\
& \cdot M^{2 p} \sum_{k=1}^{m} e^{-p \gamma\left(t-t_{k}\right)} e^{-p \alpha \sigma_{n}}\left[C_{1}+\left(c_{k}+\epsilon_{k}\right)(\mu(s))\right]+(6 m)^{p-1} \\
& \cdot M^{2 p} \sum_{k=1}^{m} e^{-p \alpha\left(t-t_{k}\right)} e^{-p \alpha \sigma_{n}}\left[C_{1}+\left(d_{k}+\epsilon_{k}\right)(\mu(s))\right] .
\end{aligned}
$$

Since $\lim _{n \rightarrow \infty} \sigma_{n}=0$, it follows that

$$
\begin{aligned}
& \mu(t) \leq 2^{p-1}\left(M_{b} E\|\varphi\|_{\mathscr{B}}\right)^{p}+2^{p-1} K_{b}^{p} E\|x(t)\|_{H}^{p} \\
& +2^{p-1} E\left\|x^{\prime}(t)\right\|_{H}^{p} \leq \widetilde{M}+12^{p-1} L_{g} \mu(t) \\
& +N_{1} M^{p} b^{p-1} L_{g} e^{-p \delta t} \int_{0}^{t} e^{p \delta s} \mu(s) d s \\
& +N_{1} M^{p} e^{-p \delta t} N_{2} \int_{0}^{t} e^{2 p \delta s} d s \\
& +N_{1} M^{p} e^{-p \delta t} N_{3} \int_{0}^{t} e^{2 p \delta s} \mu(s) d s \\
& +N_{1} C_{p} M^{p} b^{p / 2-1} e^{-p \delta t} \int_{0}^{t} e^{p \delta s} m_{f}(s) \\
& +\Theta_{f}\left(\mu(s)+b^{p-1} \int_{0}^{s} m_{h}(s, \tau) \Theta_{h}(\mu(\tau)) d \tau\right) d s \\
& +\left(10^{p-1} K_{b}^{p}+12^{p-1}\right) \\
& +m^{p-1} M^{2 p} e^{-p \delta t} N_{*}^{p} \sum_{k=1}^{m}\left(c_{k}+\epsilon_{k}\right) \mu(t)+\left(10^{p-1} K_{b}^{p}\right. \\
& \left.+12^{p-1}\right) m^{p-1} M^{2 p} e^{-p \delta t} N_{*}^{p} \sum_{k=1}^{m}\left(d_{k}+\epsilon_{k}\right) \mu(t),
\end{aligned}
$$

where

$$
\begin{aligned}
\widetilde{M} & =2^{p-1}\left(M_{b} E\|\varphi\|_{\mathscr{B}}\right)^{p}+12^{p-1} K_{b}^{p} L_{g} \\
& +N_{1} m^{p} M_{*}^{p} N_{*}^{p} M^{p} C_{1}, \\
N_{1} & =\left(10^{p-1} K_{b}^{p}+12^{p-1}\right), \\
\delta & =\min \{\alpha, \beta, \gamma\}, \\
N_{2} & =2^{p-1} \frac{1}{a^{p}} M^{p} e^{-p \delta b} M_{1}^{2 p} b^{p-1}\left\{4 ^ { p - 1 } \left[\left\|E \tilde{x}_{b}\right\|_{H}^{p}\right.\right. \\
& +C_{p} b^{p / 2-1} \int_{0}^{b} E\|\widetilde{\phi}(\eta)\|_{H}^{p} d \eta
\end{aligned}
$$

$$
\begin{aligned}
& +M^{p} e^{-p \delta b}\left[E\|\varphi(0)\|_{H}^{p}\right. \\
& \left.\left.+2^{p-1}\left[\|\phi\|_{H}^{p}+\|g(0, \varphi, \phi)\|_{H}^{p}\right]\right]\right] \\
& +M^{p} b^{p-1} L_{g} \int_{0}^{b} e^{-p \delta(b-\eta)}[\mu(\eta)+1] d \eta \\
& +C_{p} M^{p} b^{p / 2-1} \int_{0}^{b} e^{-p \delta(b-\eta)} m_{f}(\eta) \Theta_{f}(\mu(\eta) \\
& \left.+b^{p-1} \int_{0}^{\eta} m_{h}(\eta, \nu) \Theta_{h}(\mu(\nu)) d \nu\right) d \eta \\
& \left.+m^{p} M^{p} e^{-\delta p b} N_{*}^{p} C_{1}\right\},
\end{aligned}
$$

$$
\begin{aligned}
N_{3} & =2^{p-1} \frac{1}{a^{p}} M^{p} e^{-p \delta b} M_{1}^{2 p} b^{p-1} M_{*}^{p} N_{*}^{p} M^{p} m^{p-1} \sum_{k=1}^{m}\left[\left(c_{k}\right.\right. \\
& \left.\left.+\epsilon_{k}\right)+\left(d_{k}+\epsilon_{k}\right)\right] .
\end{aligned}
$$

Since $\widetilde{L}=\left[12^{p-1} L_{g}+\left(10^{p-1} K_{b}^{p}+12^{p-1}\right) m^{p-1} M^{2 p} \sum_{k=1}^{m}\left(c_{k}+\right.\right.$ $\left.\left.d_{k}+2 \epsilon_{k}\right)\right] N_{*}^{p}<1$, we obtain

$$
\begin{aligned}
& e^{\delta p t} \mu(t) \leq \frac{1}{1-\widetilde{L}}\left[\widetilde{M} N_{*}^{p}\right. \\
& +N_{1} M^{p} b^{p-1} L_{g} \int_{0}^{t} e^{p \delta s} \mu(s) d s \\
& +N_{1} M^{p} N_{2} \int_{0}^{t} e^{2 p \delta s} d s+N_{1} M^{p} N_{3} \int_{0}^{t} e^{2 \delta p s} \mu(s) d s \\
& +N_{1} C_{p} M^{p} b^{p / 2-1} \int_{0}^{t} e^{p \delta s} m_{f}(s) \Theta_{f}(\mu(s) \\
& \left.\left.+b^{p-1} \int_{0}^{s} m_{h}(s, \tau) \Theta_{h}(\mu(\tau)) d \tau\right) d s\right] .
\end{aligned}
$$

Denoting by $\zeta(t)$ the right-hand side of the above inequality, we have

$$
e^{p \delta t} \mu(t) \leq \zeta(t) \quad \forall t \in[0, b],
$$

$\zeta(0)=(1 /(1-\widetilde{L})) \widetilde{M} N_{*}^{p}$, and

$$
\begin{aligned}
& \zeta^{\prime}(t)=\frac{1}{1-\widetilde{L}}\left[N_{1} M^{p} b^{p-1} L_{g} e^{p \delta t} \mu(t)\right. \\
& +N_{1} M^{p} N_{2} e^{2 p \delta t}+N_{1} M^{p} N_{3} e^{2 p \delta t} \mu(t) \\
& +N_{1} C_{p} M^{p} b^{p / 2-1} e^{p \delta t} m_{f}(t) \Theta_{f}(\mu(t)
\end{aligned}
$$




$$
\begin{aligned}
& \left.\left.+b^{p-1} \int_{0}^{t} m_{h}(t, s) \Theta_{h}(\mu(s)) d s\right)\right] \\
& \leq \frac{1}{1-\widetilde{L}}\left[N_{1} M^{p} b^{p-1} L_{g} e^{p \delta t} e^{-p \delta t} \zeta(t)\right. \\
& +N_{1} M^{p} N_{2} e^{2 p \delta t}+N_{1} M^{p} N_{3} e^{2 p \delta t} e^{-p \delta t} \zeta(t) \\
& +N_{1} C_{p} M^{p} b^{p / 2-1} e^{p \delta t} m_{f}(t) \Theta_{f}\left(e^{-p \delta t} \zeta(t)\right. \\
& \left.\left.+b^{p-1} \int_{0}^{t} m_{h}(t, s) \Theta_{h}\left(e^{-p \delta s} \zeta(s)\right) d s\right)\right]
\end{aligned}
$$

Let $\xi(t)=e^{-p \delta t} \zeta(t)+b^{p-1} \int_{0}^{t} m_{h}(t, s) \Theta_{h}\left(e^{-p \delta s} \zeta(s)\right) d s$; then $\xi(0)=\zeta(0), e^{-p \delta t} \zeta(t) \leq \xi(t)$, and for each $t \in J$ we have

$$
\begin{aligned}
& (\xi(t))^{\prime}=-p \delta e^{-p \delta t} \zeta(t)+\zeta^{\prime}(t) e^{-p \delta t}+b^{p-1} m_{h}(t, t) \\
& \cdot \Theta_{h}\left(e^{-p \delta t} \zeta(t)\right) \leq-p \delta \xi(t) \\
& \quad+\frac{1}{1-\widetilde{L}}\left[N_{1} M^{p} b^{p-1} L_{g} \xi(t)+N_{1} M^{p} N_{2} e^{p \delta t}\right. \\
& \left.+N_{1} M^{p} N_{3} e^{p \delta t} \xi(t)\right]+\frac{1}{1-\widetilde{L}} \\
& \cdot N_{1} C_{p} M^{p} b^{p / 2-1} m_{f}(t) \Theta_{f}(\xi(t))+b^{p-1} m_{h}(t, t) \\
& \cdot \Theta_{h}(\xi(t)) \leq \widetilde{m}(t)\left[\xi(t)+1+\Theta_{f}(\xi(t))\right. \\
& \left.+\Theta_{h}(\xi(t))\right], \quad t \in J,
\end{aligned}
$$

where

$$
\begin{aligned}
& \widetilde{m}(t)=\max \{(-p \delta) \\
& +\frac{1}{1-\widetilde{L}}\left[N_{1} M^{p} b^{p-1} L_{g}+N_{1} M^{2} N_{3} e^{p \delta t}\right], \frac{1}{1-\widetilde{L}} \\
& \cdot N_{1} M^{2} N_{2} e^{p \delta t}, \frac{1}{1-\widetilde{L}} \\
& \left.\cdot K_{b} N_{1} C_{p} M^{p} b^{p / 2-1} m_{f}(t), b^{p-1} m_{h}(t, t)\right\} .
\end{aligned}
$$

This implies that

$$
\int_{\xi(0)}^{\xi(t)} \frac{d \vartheta}{1+\vartheta+\Theta_{f}(\vartheta)+\Theta_{h}(\vartheta)} \leq \int_{0}^{b} \widetilde{m}(t) d t<\infty
$$

This inequality shows that there is a constant $\widetilde{K}$ such that $\xi(t) \leq \widetilde{K}, t \in J$, and hence $\|x\|_{\mathscr{P} \mathscr{C}^{1}} \leq \mu(t) \leq e^{-p \delta t} \zeta(t) \leq \xi(t) \leq$ $\widetilde{K}$, where $\widetilde{K}$ depends only on $M, \delta, p, C_{p}, K_{b}$, and $b$ and on the functions $m_{f}(\cdot), m_{h}(\cdot, \cdot), \Theta_{f}(\cdot)$ and $\Theta_{h}(\cdot)$. This indicates that $G$ is bounded on $J$. Consequently, by Lemma 13, we deduce that
$\Lambda_{n}+\Upsilon_{n}$ has a fixed point $x_{n} \in \mathscr{B}_{b}^{0}$, which is a mild solution of problem (27). Then, we have

$$
\begin{aligned}
x_{n}(t)= & \int_{0}^{t} C(t-s) g\left(s, \bar{x}_{n, s}, \bar{x}_{n}^{\prime}(s)\right) d s \\
& +\int_{0}^{t} S(t-s) B u_{\bar{x}_{n}}^{a}(s) d s \\
& +\int_{0}^{t} S(t-s) f(s) d w(s) \\
& +\sum_{0<t_{k}<t} C\left(t-t_{k}\right) C\left(\sigma_{n}\right) I_{k}\left(\bar{x}_{n, t_{k}}, \bar{x}_{n}^{\prime}\left(t_{k}\right)\right) \\
& +\sum_{0<t_{k}<t} S\left(t-t_{k}\right) S\left(\sigma_{n}\right) J_{k}\left(\bar{x}_{n, t_{k}}, \bar{x}_{n}^{\prime}\left(t_{k}\right)\right)
\end{aligned}
$$

for $t \in[0, b]$, and some $f_{n} \in S_{F, \bar{x}_{n}}$.

Next we will show that the set $\left\{x_{n}: n \in \mathbb{N}\right\}$ is relatively compact in $\mathscr{B}_{b}^{0}$. We consider the decomposition $x_{n}=x_{n}^{1}+x_{n}^{2}$, where

$$
\begin{aligned}
x_{n}^{1}(t)= & \int_{0}^{t} C(t-s) g\left(s, \bar{x}_{n, s}, \bar{x}_{n}^{\prime}(s)\right) d s \\
& +\int_{0}^{t} S(t-s) B u_{\bar{x}_{n}}^{a}(s) d s \\
& +\int_{0}^{t} S(t-s) f(s) d w(s)
\end{aligned}
$$

for some $f_{n} \in S_{F, \bar{x}_{n}}$, and

$$
\begin{aligned}
x_{n}^{2}(t)= & \sum_{0<t_{k}<t} C\left(t-t_{k}\right) C\left(\sigma_{n}\right) I_{k}\left(\bar{x}_{n, t_{k}}, \bar{x}_{n}^{\prime}\left(t_{k}\right)\right) \\
& +\sum_{0<t_{k}<t} S\left(t-t_{k}\right) S\left(\sigma_{n}\right) J_{k}\left(\bar{x}_{n, t_{k}}, \bar{x}_{n}^{\prime}\left(t_{k}\right)\right) .
\end{aligned}
$$

Step 4. $\left\{x_{n}^{1}(t): n \in \mathbb{N}\right\}$ is relatively compact in $\mathscr{B}_{b}^{0}$.

(1) $\left\{x_{n}^{1}: n \in \mathbb{N}\right\}$ is equicontinuous on $J$.

For $\varepsilon>0, x_{n} \in B_{r}\left(0, \mathscr{B}_{b}^{0}\right)$, there exists a constant $0<\tilde{\eta}<\varepsilon$ such that, for all $t \in(0, b]$ and $\xi \in(0, \tilde{\eta})$ with $t+\xi \leq b$, we have

$$
\begin{aligned}
& E\left\|x_{n}^{1}(t+\xi)-x_{n}^{1}(t)\right\|_{H}^{p} \leq 6^{p-1} E \| \int_{t}^{t+\xi} C(t+\xi-s) \\
& \cdot g\left(s, \bar{x}_{n, s}, \bar{x}_{n}^{\prime}(s)\right) d s \|_{H}^{p} \\
& +6^{p-1} E \| \int_{0}^{t}[C(t+\xi-s)-C(t-s)] \\
& \cdot g\left(s, \bar{x}_{n, s}, \bar{x}_{n}^{\prime}(s)\right) d s\left\|_{H}^{p}+6^{p-1}\right\| \int_{t}^{t+\xi} S(t+\xi-s)
\end{aligned}
$$


Journal of Function Spaces

21

$$
\begin{aligned}
& \cdot B u_{\bar{x}_{n}}^{a}(s) d s\left\|_{H}^{p}+6^{p-1}\right\| \int_{0}^{t}[S(t+\xi-s) \\
& -S(t-s)] B u_{\bar{x}_{n}}^{a}(s) d s\left\|_{H}^{p}+6^{p-1} E\right\|_{t}^{t+\xi} S(t+\xi \\
& -s) f_{n}(s) d w(s) \|_{H}^{p} \\
& +6^{p-1} E \| \int_{0}^{t}[S(t+\xi-s)-S(t-s)] \\
& \cdot f_{n}(s) d w(s) \|_{H}^{p} \\
& \leq 6^{p-1} \xi{ }^{p-1} M^{p} \int_{t}^{t+\xi} e^{-p \alpha(t+\xi-s)} L_{G}\left(r^{*}+r+1\right) d s \\
& +\int_{t}^{p-1} b^{p-1} \int_{0}^{t}\|C(t+\xi-s)-C(t-s)\|_{H}^{p} L_{G}\left(r^{*}\right. \\
& +M_{2} d s+6^{p-1} C_{p} M^{p} \Theta\left(t+r_{f}^{* *}\right)\left[\frac{2 \delta(p-1)}{p-2}\right]^{1-p / 2} \\
& +r+1) d s \\
& +6^{p-1} M^{p} M_{1}^{p} \xi^{p-1} \int_{t}^{t+\xi} e^{-\beta(t+\xi-s)} M_{2} d s \\
& +6^{p-1} M_{1}^{p} b^{p-1} \int_{0}^{t}\left[\|S(t+\xi-s)-S(t-s)\|_{H}^{p}\right]
\end{aligned}
$$

Using the compact operator property, we can choose $\xi \in(0, t)$ such that

$$
\begin{aligned}
& 6^{p-1} \xi^{p-1} M^{p} \int_{t}^{t+\xi} e^{-p \alpha(t+\xi-s)} L_{G}\left(r^{*}+r+1\right) d s<\frac{\varepsilon}{6}, \\
& 6^{p-1} b^{p-1} \int_{0}^{t}\|C(t+\xi-s)-C(t-s)\|_{H}^{p} \\
& \cdot L_{G}\left(r^{*}+r+1\right) d s<\frac{\varepsilon}{6}, \\
& 6^{p-1} M^{p} M_{1}^{p} \xi^{p-1} \int_{t}^{t+\xi} e^{-\beta(t+\xi-s)} M_{2} d s<\frac{\varepsilon}{6}, \\
& 6^{p-1} M_{1}^{p} b^{p-1} \int_{0}^{t}\left[\|S(t+\xi-s)-S(t-s)\|_{H}^{p}\right] M_{2} d s \\
& <\frac{\varepsilon}{6},
\end{aligned}
$$

$$
\begin{aligned}
& 6^{p-1} C_{p} M^{p} \Theta_{f}\left(r^{* *}\right)\left[\frac{2 \delta(p-1)}{p-2}\right]^{1-p / 2} \\
& \cdot \int_{t}^{t+\xi} e^{-\beta(t+\xi-s)} m_{f}(s) d s<\frac{\varepsilon}{6}, \\
& 6^{p-1} C_{p} \Theta_{f}\left(r^{* *}\right) \\
& \cdot\left[\int_{0}^{t}\left[\|S(t+\xi-s)-S(t-s)\|_{H}^{p} m_{f}(s)\right]^{2 / p} d s\right]^{p / 2} \\
& <\frac{\varepsilon}{6} .
\end{aligned}
$$

By (83) one has

$$
E\left\|x_{n}^{1}(t+\xi)-x_{n}^{1}(t)\right\|_{H}^{p}<\varepsilon .
$$

Therefore, $\left\{x_{n}^{1}(t): n \in \mathbb{N}\right\}$ is equicontinuous for $t \in(0, b]$. Clearly $\left\{x_{n}^{1}(0): n \in \mathbb{N}\right\}$ is equicontinuous.

(2) $\left\{x_{n}^{1}(t): n \in \mathbb{N}\right\}$ is relatively compact in $H$.

Let $t \in(0, b], \varepsilon>0, x_{n} \in B_{r}(0, \mathscr{B} \mathscr{P} \mathscr{C})$; there exists $\xi \epsilon$ $(0, t)$ such that

$$
\begin{aligned}
& E\left\|x_{n}^{1}(t)-x_{n}^{\xi}(t)\right\|_{H}^{p} \\
& \leq 3^{p-1}\left\|\int_{t-\xi}^{t} C(t-s) g\left(s, \bar{x}_{n, s}, \bar{x}_{n}^{\prime}(s)\right) d s\right\|_{H}^{p} \\
& +3^{p-1}\left\|\int_{t-\xi}^{t} S(t-s) B u_{\bar{x}_{n}}^{a}(s) d s\right\|_{H}^{p} \\
& +3^{p-1}\left\|\int_{t-\xi}^{t} S(t-s) f_{n}(s) d w(s)\right\|_{H}^{p} \\
& \quad \leq 3^{p-1} \xi^{p-1} M^{p} L_{G}\left(r^{*}+r+1\right) \int_{t-\xi}^{t} e^{-p \alpha(t-s)} d s \\
& +3^{p-1} M^{p} M_{1}^{p} \beta^{1-p} \int_{t-\xi}^{t} e^{-\beta(t-s)} M_{2} d s \\
& +3^{p-1} C_{p} M^{p} \Theta_{f}\left(r^{* *}\right)\left[\frac{2 \beta(p-1)}{p-2}\right]^{1-p / 2} \\
& \quad \int_{t}^{t-\xi} e^{-\beta(t-s)} m_{f}(s) d s<\varepsilon,
\end{aligned}
$$

where

$$
\begin{aligned}
x_{n}^{\xi}(t)= & \int_{0}^{t-\xi} C(t-s) g\left(s, \bar{x}_{n, s}, \bar{x}_{n}^{\prime}(s)\right) d s \\
& +\int_{0}^{t-\xi} S(t-s) B u_{\bar{x}_{n}}^{a}(s) d s \\
& +\int_{0}^{t-\xi} S(t-s) f_{n}(s) d w(s)
\end{aligned}
$$

for some $f_{n} \in S_{F, \bar{x}_{n}}$. By the compactness of $C(t), S(t)$ for $t>0$, we see that the set $\left\{x_{n}^{\xi}(t): n \in \mathbb{N}\right\}$ is relatively compact in 
$H$. Combining the above inequality, one has $\left\{x_{n}^{1}(t): n \in \mathbb{N}\right\}$ which is relatively compact in $H$.

Step 5. $\left\{x_{n}^{2}(t): n \in \mathbb{N}\right\}$ is relatively compact in $\mathscr{B}_{b}^{0}$.

(1) $\left\{x_{n}^{2}: n \in \mathbb{N}\right\}$ is equicontinuous on $J$.

For any $\varepsilon>0,0<t<b$. Since $C\left(\sigma_{n}\right), S\left(\sigma_{n}\right)$ are compact operators, we find that the sets $W_{1}=\left\{C\left(\sigma_{n}\right) I_{k}\left(\bar{x}_{n, t_{k}}, \bar{x}_{n}^{\prime}\left(t_{k}\right)\right)\right.$ : $\left.x_{n} \in B_{r}\left(0, \mathscr{B}_{b}^{0}\right)\right\}$ and $W_{2}=\left\{S\left(\sigma_{n}\right) J_{k}\left(\bar{x}_{n, t_{k}}, \bar{x}_{n}^{\prime}\left(t_{k}\right)\right): x_{n} \in\right.$ $\left.B_{r}\left(0, \mathscr{B}_{b}^{0}\right)\right\}$ are relatively compact in $H$. From the strong continuity of $(C(t))_{t \geq 0}$, for $\varepsilon>0$, we can choose $0<\widetilde{\eta}<b-t$ such that

$$
\begin{array}{cc}
E\left\|(C(t+\xi)-C(t)) v_{1}\right\|_{H}^{p}<\frac{\varepsilon}{2(2 m)^{p-1}}, \quad v_{1} \in W_{1}, \\
E\left\|(S(t+\xi)-S(t)) v_{2}\right\|_{H}^{p}<\frac{\varepsilon}{2(2 m)^{p-1}}, \quad v_{2} \in W_{2}
\end{array}
$$

when $|\xi|<\widetilde{\eta}$. For each $x_{n} \in B_{r}\left(0, \mathscr{B}_{b}^{0}\right), t \in(0, b)$ is fixed and $t \in\left[t_{k}, t_{k+1}\right]$, such that

$$
\begin{aligned}
& E\left\|\left[\widehat{x_{n}^{2}}\right]_{k}(t+\xi)-\left[\widehat{x_{n}^{2}}\right]_{k}(t)\right\|_{H}^{p} \\
& \quad \leq 2^{p-1} E \| \sum_{0<t_{k}<t}\left[C\left(t+\xi-t_{k}\right)-C\left(t-t_{k}\right)\right] C\left(\sigma_{n}\right)
\end{aligned}
$$

$$
\begin{aligned}
& \cdot I_{k}\left(\bar{x}_{n, t_{k}}, \bar{x}_{n}^{\prime}\left(t_{k}\right)\right) \|_{H}^{p} \\
& +2^{p-1} E \| \sum_{0<t_{k}<t}\left[S\left(t+\xi-t_{k}\right)-S\left(t-t_{k}\right)\right] S\left(\sigma_{n}\right) \\
& \cdot J_{k}\left(\bar{x}_{n, t_{k}}, \bar{x}_{n}^{\prime}\left(t_{k}\right)\right) \|_{H}^{p} \leq(2 m)^{p-1} \\
& \cdot \sum_{k=1}^{m} E \|\left[C\left(t+\xi-t_{k}\right)-C\left(t-t_{k}\right)\right] C\left(\sigma_{n}\right) \\
& \cdot I_{k}\left(\bar{x}_{n, t_{k}}, \bar{x}_{n}^{\prime}\left(t_{k}\right)\right) \|_{H}^{p}+(2 m)^{p-1} \\
& \cdot \sum_{k=1}^{m} E \|\left[S\left(t+\xi-t_{k}\right)-S\left(t-t_{k}\right)\right] S\left(\sigma_{n}\right) \\
& \cdot J_{k}\left(\bar{x}_{n, t_{k}}, \bar{x}_{n}^{\prime}\left(t_{k}\right)\right) \|_{H}^{p}<\varepsilon .
\end{aligned}
$$

As $\xi \rightarrow 0$ and $\varepsilon$ is sufficiently small, the right-hand side of the above inequality tends to zero independently of $x_{n}$, so $\left[\widehat{x_{n}^{2}}\right]_{k}$, $k=1,2, \ldots, m$, are equicontinuous.

(2) $\left\{x_{n}^{2}(t): n \in \mathbb{N}\right\}$ is relatively compact in $H$.

For $t \in\left[t_{k}, t_{k+1}\right], k=1, \ldots, m$, and $x_{n} \in B_{r}\left(0, \mathscr{B}_{b}^{0}\right)$, we have that there exists $r^{\prime}>0$ such that

$$
\begin{aligned}
& {\left[\widehat{x_{n}^{2}}\right]_{k}(t)} \\
& \epsilon\left\{\begin{array}{lr}
\sum_{i=1}^{k} C\left(t-t_{i}\right) C\left(\sigma_{n}\right) I_{i}\left(\bar{x}_{n, t_{i}} \bar{x}_{n}^{\prime}\left(t_{i}\right)\right)+\sum_{i=1}^{k} S\left(t-t_{i}\right) S\left(\sigma_{n}\right) I_{i}\left(\bar{x}_{n, t_{i}}, \bar{x}_{n}^{\prime}\left(t_{i}\right)\right), & t \in\left(t_{k}, t_{k+1}\right), x_{n} \in B_{r^{\prime}}\left(0, \mathscr{B}_{b}^{0}\right), \\
\sum_{i=1}^{k} C\left(t_{k+1}-t_{i}\right) C\left(\sigma_{n}\right) I_{i}\left(\bar{x}_{n, t_{i}}, \bar{x}_{n}^{\prime}\left(t_{i}\right)\right)+\sum_{i=1}^{k} S\left(t_{k+1}-t_{i}\right) S\left(\sigma_{n}\right) J_{i}\left(\bar{x}_{n, t_{i}}, \bar{x}_{n}^{\prime}\left(t_{i}\right)\right), & t=t_{k+1}, x_{n} \in B_{r^{\prime}}\left(0, \mathscr{B}_{b}^{0}\right), \\
\sum_{i=1}^{k-1} C\left(t_{k}-t_{i}\right) C\left(\sigma_{n}\right) I_{i}\left(\bar{x}_{n, t_{i}} \bar{x}_{n}^{\prime}\left(t_{i}\right)\right)+C\left(\sigma_{n}\right) I_{i}\left(\bar{x}_{n, t_{i}}, \bar{x}_{n}^{\prime}\left(t_{i}\right)\right)+\sum_{i=1}^{k-1} S\left(t_{k}-t_{i}\right) S\left(\sigma_{n}\right) J_{i}\left(\bar{x}_{n, t_{i}}, \bar{x}_{n}^{\prime}\left(t_{i}\right)\right)+S\left(\sigma_{n}\right) J_{i}\left(\bar{x}_{n, t_{i}}, \bar{x}_{n}^{\prime}\left(t_{i}\right)\right) & t=t_{k}, x_{n} \in B_{r^{\prime}}\left(0, \mathscr{B}_{b}^{0}\right),
\end{array}\right.
\end{aligned}
$$

where $B_{r^{\prime}}\left(0, \mathscr{B}_{b}^{0}\right)$ is a closed ball of radius $r^{\prime}$. One has $\left[\widehat{x_{n}^{2}}\right]_{k}(t), k=1,2, \ldots, m$, which is relatively compact for every $t \in\left[t_{k}, t_{k+1}\right]$, and $\left\{x_{n}^{2}(t): n \in \mathbb{N}\right\}$ is relatively compact in $H$.

These facts imply the relatively compact of $\left\{x_{n}: n \in \mathbb{N}\right\}$ in $\mathscr{B}_{b}^{0}$. Therefore, without loss of generality, we may suppose that

$$
x_{n} \longrightarrow x_{*} \in \mathscr{B}_{b}^{0} \text { as } n \longrightarrow \infty \text {. }
$$

Obviously, $x_{*} \in \mathscr{B}_{b}^{0}$; taking limits in (79) one has

$$
\begin{aligned}
x_{*}(t)= & \int_{0}^{t} C(t-s) g\left(s, \bar{x}_{*, s}, \bar{x}_{*}^{\prime}(s)\right) d s \\
& +\int_{0}^{t} S(t-s) B u_{\bar{x}_{*}}^{a}(s) d s
\end{aligned}
$$

$$
\begin{aligned}
& +\int_{0}^{t} S(t-s) f_{*}(s) d w(s) \\
& +\sum_{0<t_{k}<t} C\left(t-t_{k}\right) I_{k}\left(\bar{x}_{*, t_{k}}, \bar{x}_{*}^{\prime}\left(t_{k}\right)\right) \\
& +\sum_{0<t_{k}<t} S\left(t-t_{k}\right) J_{k}\left(\bar{x}_{*, t_{k}}, \bar{x}_{*}^{\prime}\left(t_{k}\right)\right)
\end{aligned}
$$

for $t \in[0, b]$, and some $f_{*} \in S_{F, \bar{x}_{*}}$, which implies that $x_{*}$ is a mild solution of the problem (1) and the proof of Theorem 16 is complete. 


\section{Approximate Controllability of Impulsive Stochastic Control System}

In this section, we present our main result on approximate controllability of system (1). To do this, we also need the following assumptions:

(B1) The function $g: J \times \mathscr{B} \times H \rightarrow H$ is continuous and there exists a constant $\widetilde{C}_{1}>0$ such that

$$
E\|g(t, \psi, y)\|_{H}^{p} \leq \widetilde{C}_{1}
$$

for $0 \leq t \leq b,(\psi, y) \in \mathscr{B} \times H$.

(B2) There exists a constant $\widetilde{C}_{2}>0$ such that

$$
\|F(t, \psi, y, z)\|_{H}^{p} \leq \widetilde{C}_{2}
$$

for $0 \leq t \leq b,(\psi, y, z) \in \mathscr{B} \times H \times H$, where

$\|F(t, \psi, y, z)\|_{H}^{p}=\sup \left\{E\|f\|_{H}^{p}: f \in F(t, \psi, y, z)\right\}$.

Theorem 17. Assume that assumptions of Theorem 16 hold and, in addition, hypotheses (S1), (B1), and (B2) are satisfied. Then system (1) is approximately controllable on $\mathrm{J}$.

Proof. Let $x^{a}(\cdot)$ be a fixed point of $\Phi$ in $\mathscr{B}_{b}^{0}$. By Theorem 16, any fixed point of $\Phi$ is a mild solution of system (1). This means that there is $x^{a} \in \Phi\left(x^{a}\right)$; that is, there is $f \in S_{F, \bar{x}^{a}}$ such that

$$
\begin{aligned}
x^{a}(t)= & \int_{0}^{t} C(t-s) g\left(s, \bar{x}_{s}^{a},\left(\bar{x}^{a}\right)^{\prime}(s)\right) d s \\
& +\int_{0}^{t} S(t-s) B u_{\bar{x}}^{a}(s) d s \\
& +\int_{0}^{t} S(t-s) f(s) d w(s) \\
& +\sum_{0<t_{k}<t} C\left(t-t_{k}\right) I_{k}\left(\bar{x}_{t_{k}}^{a},\left(\bar{x}^{a}\right)^{\prime}\left(t_{k}\right)\right) \\
& +\sum_{0<t_{k}<t} S\left(t-t_{k}\right) J_{k}\left(\bar{x}_{t_{k}}^{a},\left(\bar{x}^{a}\right)^{\prime}\left(t_{k}\right)\right), \quad t \in J
\end{aligned}
$$

where

$$
\begin{gathered}
u_{x}^{a}(t)=B^{*} S^{*}(b-t)\left(a I+\Gamma_{0}^{b}\right)^{-1}\left[E \tilde{x}_{b}\right. \\
+\int_{0}^{b} \widetilde{\phi}(s) d w(s)-C(b) \varphi(0)
\end{gathered}
$$

$$
\begin{aligned}
& -S(b)[\phi-g(0, \varphi, \phi)]-B^{*} S^{*}(b-t) \\
& \cdot \int_{0}^{b}\left(a I+\Gamma_{s}^{b}\right)^{-1} C(b-s) g\left(s, \bar{x}_{s}^{a},\left(\bar{x}^{a}\right)^{\prime}(s)\right) d s \\
& -B^{*} S^{*}(b-t) \int_{0}^{b}\left(a I+\Gamma_{s}^{b}\right)^{-1} S(b-s) f(s) d w(s) \\
& -B^{*} S^{*}(b-t)\left(a I+\Gamma_{s}^{b}\right)^{-1} \\
& \cdot \sum_{k=1}^{m} C\left(b-t_{k}\right) I_{k}\left(\bar{x}_{t_{k}}^{a},\left(\bar{x}^{a}\right)^{\prime}\left(t_{k}\right)\right)-B^{*} \delta_{\alpha}^{*}(b-t) \\
& \cdot\left(a I+\Gamma_{s}^{b}\right)^{-1} \\
& \cdot \sum_{k=1}^{m} S\left(b-t_{k}\right) J_{k}\left(\bar{x}_{t_{k}}^{a},\left(\bar{x}^{a}\right)^{\prime}\left(t_{k}\right)\right), \quad t \in J,
\end{aligned}
$$

and by using the stochastic Fubini theorem, it is easy to see that

$$
\begin{aligned}
& x^{a}(b)=\int_{0}^{b} C(b-s) g\left(s, \bar{x}_{s}^{a},\left(\bar{x}^{a}\right)^{\prime}(s)\right) d s \\
& +\int_{0}^{b} S(b-s) B u_{\bar{x}}^{a}(s) d s+\int_{0}^{b} S(b-s) \\
& \cdot f(s) d w(s)+\sum_{0<t_{k}<b} C\left(b-t_{k}\right) \\
& \cdot I_{k}\left(\bar{x}_{t_{k}}^{a},\left(\bar{x}^{a}\right)^{\prime}\left(t_{k}\right)\right)+\sum_{0<t_{k}<b} S\left(b-t_{k}\right) \\
& \cdot J_{k}\left(\bar{x}_{t_{k}}^{a},\left(\bar{x}^{a}\right)^{\prime}\left(t_{k}\right)\right)=x_{b}-a\left(a I+\Gamma_{0}^{b}\right)^{-1}\left[E \tilde{x}_{b}\right. \\
& +\int_{0}^{b} \tilde{\phi}(s) d w(s)-C(b) \varphi(0)-S(b) \\
& \cdot[\phi-g(0, \varphi, \phi)]]-a \int_{0}^{b}\left(a I+\Gamma_{s}^{b}\right)^{-1} C(b-s) \\
& \cdot g\left(s, \bar{x}_{s}^{a},\left(\bar{x}^{a}\right)^{\prime}(s)\right) d s-a \int_{0}^{b}\left(a I+\Gamma_{s}^{b}\right)^{-1} \\
& \cdot S(b-s) f(s) d w(s)-a\left(a I+\Gamma_{s}^{b}\right)^{-1} \\
& \cdot \sum_{k=1}^{m} C\left(b-t_{k}\right) I_{k}\left(\bar{x}_{t_{k}}^{a},\left(\bar{x}^{a}\right)^{\prime}\left(t_{k}\right)\right)-a\left(a I+\Gamma_{s}^{b}\right)^{-1} \\
& \cdot \sum_{k=1}^{m} S\left(b-t_{k}\right) J_{k}\left(\bar{x}_{t_{k}}^{a},\left(\bar{x}^{a}\right)^{\prime}\left(t_{k}\right)\right) .
\end{aligned}
$$

By conditions (B1) and (B2), we get that the sequences $\left\{g\left(s, \bar{x}_{s}^{a},\left(\bar{x}^{a}\right)^{\prime}(s)\right)\right\}$ and $\{f(s)\}$ are uniformly bounded on $J$. Thus there are subsequences, still denoted by $\left\{g\left(s, \bar{x}_{s}^{a},\left(\bar{x}^{a}\right)^{\prime}(s)\right)\right\}$ and $\{f(s)\}$ that converge weakly to, say, $g(s)$ in $H$ and $f^{* *}(s)$ in $L(K, H)$, respectively. The compactness of $S(t), t>0$, implies that $S(b-s)\left[g\left(s, \bar{x}_{s}^{a},\left(\bar{x}^{a}\right)^{\prime}(s)\right)-\right.$ 
$g(s)] \rightarrow 0, S(b-s)\left[f(s)-f^{* *}(s)\right] \rightarrow 0$. On the other hand, by Lemma 9, for all $t \in J, a\left(a I+\Gamma_{t}^{b}\right)^{-1} \rightarrow 0$ strongly as $a \rightarrow 0^{+}$and $\left\|a\left(a I+\Gamma_{t}^{b}\right)^{-1}\right\| \leq 1$. Therefore, by the Lebesque dominated convergence theorem it follows that

$$
\begin{aligned}
& E\left\|x^{a}(b)-x_{b}\right\|_{H}^{p} \leq 8^{p-1} E \| a\left(a I+\Gamma_{0}^{b}\right)^{-1} E \tilde{x}_{b}-C(b) \\
& \cdot \varphi(0)-S(b)[\phi-g(0, \varphi, \phi)] \|_{H}^{p} \\
& +8^{p-1} E\left(\int_{0}^{b}\left\|a\left(a I+\Gamma_{0}^{b}\right)^{-1} \tilde{\phi}(s)\right\|_{H}^{2} d s\right)^{p / 2} \\
& +8^{p-1}\left(\int_{0}^{b} \| a\left(a I+\Gamma_{s}^{b}\right)^{-1} C(b-s)\right. \\
& \left.\cdot\left[g\left(s, \bar{x}_{s}^{a},\left(\bar{x}^{a}\right)^{\prime}(s)\right)-g(s)\right] \|_{H} d \eta\right)^{p} \\
& +8^{p-1} E\left(\int_{0}^{b}\left\|a\left(a I+\Gamma_{s}^{b}\right)^{-1} C(b-s) g(s)\right\|_{H} d s\right)^{p} \\
& +8^{p-1} E\left(\int_{0}^{b} \| a\left(a I+\Gamma_{s}^{b}\right)^{-1} S(b-s)\right. \\
& \left.\cdot\left[f(s)-f^{* *}(s)\right] \|_{H}^{2} d s\right)^{p / 2} \\
& +8^{p-1} E\left(\int_{0}^{b} \| a\left(a I+\Gamma_{s}^{b}\right)^{-1} S(b-s)\right. \\
& \left.\cdot f^{* *}(s) \|_{H}^{2} d s\right)^{p / 2}+8^{p-1} E\left(\| a\left(a I+\Gamma_{s}^{b}\right)^{-1}\right. \\
& \left.\cdot \sum_{k=1}^{m} C\left(b-t_{k}\right) I_{k}\left(\bar{x}_{t_{k}}^{a},\left(\bar{x}^{a}\right)^{\prime}\left(t_{k}\right)\right) \|_{H}\right)^{p} \\
& +8^{p-1} E\left(\| a\left(a I+\Gamma_{s}^{b}\right)^{-1} \sum_{k=1}^{m} S\left(b-t_{k}\right)\right. \\
& \left.\cdot J_{k}\left(\bar{x}_{t_{k}}^{a},\left(\bar{x}^{a}\right)^{\prime}\left(t_{k}\right)\right) \|_{H}\right)^{p} \longrightarrow 0 \quad \text { as } a \longrightarrow 0^{+} .
\end{aligned}
$$

So $x^{a}(b) \rightarrow x_{b}$ holds, which shows that system (1) is approximately controllable and the proof is complete.

\section{Example}

Consider the following impulsive partial stochastic neutral differential inclusions of the form

$$
\begin{aligned}
& d\left[\frac{\partial}{\partial t} z(t, x)-\int_{-\infty}^{t} b_{1}(s-t) z(s, x) d s\right. \\
& \left.-b_{2}(s) z^{\prime}(t, x)\right] \in \frac{\partial^{2}}{\partial^{2} t} z(t, x) d t+\widetilde{u}(t, x) d t
\end{aligned}
$$

$$
\begin{gathered}
+\left[\int_{-\infty}^{t} \Phi_{1}(t, t-s, x, z(s, x)) d s+\varsigma_{1}(t) z^{\prime}(t, x)\right. \\
+\int_{0}^{t} \int_{-\infty}^{s} \varsigma_{2}(t) \Phi_{2}(s, \tau-s, x, z(\tau, x)) d \tau d s \\
\left.+\int_{0}^{t} \varsigma_{3}(s) z^{\prime}(s, x) d s\right] d w(t), \\
z(t, 0)=z(t, \pi)=0, \quad 0 \leq t \leq b, \\
z(t, x)=\varphi(t, x), \quad 0 \leq t \leq b, 0 \leq x \leq \pi, t \neq t_{k}, \\
\frac{\partial}{\partial t} z(0, x)=\phi(x), \\
\Delta z\left(t_{k}, x\right)=\int_{-\infty}^{t_{k}} \eta_{k}\left(s-t_{k}\right) z(s, x) d s+k_{1}\left(t_{k}\right) \\
\cdot z^{\prime}\left(t_{k}, x\right), \quad k=1,2, \ldots, m, \\
\Delta z^{\prime}\left(t_{k}, x\right)=\int_{-\infty}^{t_{k}} \tilde{\eta}_{k}\left(s-t_{k}\right) z(s, x) d s+k_{2}\left(t_{k}\right) \\
\cdot z^{\prime}\left(t_{k}, x\right), \quad k=1,2, \ldots, m,
\end{gathered}
$$

where $\left(t_{k}\right)_{k} \in \mathbb{N}$ is a strictly increasing sequence of positive numbers and $\widetilde{u}(\cdot)$ is a real function of bounded variation on $[0, b] . w(t)$ denotes a standard cylindrical Wiener process in $H$ defined on a stochastic space $(\Omega, \mathscr{F}, P)$.

Let $H=L^{2}([0, \pi])$ with the norm $\|\cdot\|_{H}$ and define the operator $A$ by $A \omega=\omega^{\prime \prime}$ with the domain

$$
\begin{aligned}
& D(A):=\{\omega(\cdot) \\
& \quad \in H: \omega, \omega^{\prime} \text { are absolutely continuous, } \omega^{\prime \prime} \\
& \quad \in H, \omega(0)=\omega(\pi)=0\} .
\end{aligned}
$$

It is well known that $A$ is the infinitesimal generator of a strongly continuous cosine family $\{C(t): t \in \mathbb{R}\}$ in $H$ and is given by

$$
C(t) \omega=\sum_{n=1}^{\infty} \cos (n t)\left\langle\omega, e^{n}\right\rangle e^{n}, \quad \omega \in H,
$$

where $e^{n}(\theta)=\sqrt{2 / \pi} \sin n \theta, i=1,2, \ldots$, is the orthogonal set of eigenvalues of $A$. The associated sine family $S(t), t>0$, is compact and is given by

$$
S(t) \omega=\sum_{n=1}^{\infty} \frac{1}{n} \sin (n t)\left\langle\omega, e^{n}\right\rangle e^{n}, \quad \omega \in H .
$$

Additionally, we will assume the following:

(i) The functions $b_{i}: \mathbb{R} \rightarrow \mathbb{R}, i=1,2$, are continuous, and $\widetilde{L}_{1}=\left(\int_{-\infty}^{0}\left(\left(b_{1}(s)\right)^{2} / \widetilde{h}(s)\right) d s\right)^{1 / 2}<\infty$. 
(ii) The functions $\omega_{i}: \mathbb{R}^{4} \rightarrow \mathbb{R}, i=1,2$, are continuous and there exist continuous functions $a_{j}: \mathbb{R} \rightarrow \mathbb{R}$, $j=1,2,3,4$, such that

$$
\begin{array}{ll}
\left|\omega_{1}(t, s, x, y)\right| \leq a_{1}(t) a_{2}(s)|y|, & (t, s, x, y) \in \mathbb{R}^{4}, \\
\left|\omega_{2}(t, s, x, y)\right| \leq a_{3}(t) a_{4}(s)|y|, & (t, s, x, y) \in \mathbb{R}^{4}
\end{array}
$$

with $\widehat{L}_{1}=\left(\int_{-\infty}^{0}\left(\left(a_{2}(s)\right)^{2} / \tilde{h}(s)\right) d s\right)^{1 / 2}<\infty, \widehat{L}_{2}=$ $\left(\int_{-\infty}^{0}\left(\left(a_{4}(s)\right)^{2} / \widetilde{h}(s)\right) d s\right)^{1 / 2}<\infty$.

(iii) The functions $\varsigma_{i}, k_{i}: \mathbb{R} \rightarrow \mathbb{R}, i=1,2$, are continuous.

(iv) The functions $\eta_{k}, \widetilde{\eta}_{k}: \mathbb{R} \rightarrow \mathbb{R}, k=1,2, \ldots, m$, are continuous, $\vartheta_{k}=\left(\int_{-\infty}^{0}\left(\left(\eta_{k}(s)\right)^{2} / \tilde{h}(s)\right) d s\right)^{1 / 2}<\infty$, and $\widetilde{\vartheta}_{k}=\left(\int_{-\infty}^{0}\left(\left(\widetilde{\eta}_{k}(s)\right)^{2} / \widetilde{h}(s)\right) d s\right)^{1 / 2}<\infty$ for every $k=$ $1,2, \ldots, m$.

Take $\mathscr{B}=\mathscr{P} \mathscr{C}_{0} \times L^{2}(h, H)$ which is the space introduced in Example 2. Set $\varphi(\theta)(x)=\varphi(\theta, x) \in \mathscr{B}$, defining the maps $g:[0, b] \times \mathscr{B} \times H \rightarrow H, F:[0, b] \times \mathscr{B} \times H \times H \rightarrow \mathscr{P}(H)$ by

$$
\begin{aligned}
& g\left(t, \psi, \psi^{\prime}\right)(x) \\
& =\int_{-\infty}^{t} b_{1}(s) \psi(s, x) d s+b_{2}(t) \psi^{\prime}(t, x), \\
& f\left(t, \psi, \psi^{\prime}, B_{1} \psi\right)(x) \\
& =\int_{-\infty}^{0} \omega_{1}(t, s, x, \psi(s, x)) d s+\varsigma_{1}(t) \psi^{\prime}(t, x) \\
& \quad+B_{1} \psi(x), \\
& B_{1} \psi(x) \\
& \quad \int_{0}^{t} \int_{-\infty}^{0} \varsigma_{2}(t) \Phi_{2}(s, \tau, x, \psi(\tau, x)) d \tau d s \\
& \quad+\int_{0}^{t} \varsigma_{3}(s) \psi^{\prime}(s, x) d s, \\
& I_{k}\left(\psi, \psi^{\prime}\right)(x) \\
& =\int_{-\infty}^{0} \eta_{k}(s) \psi(s, x) d s+k_{1}\left(t_{k}\right) \psi^{\prime}\left(t_{k}, x\right), \\
& J_{k}\left(\psi, \psi^{\prime}\right)(x) \\
& =\int_{-\infty}^{0} \eta_{k}(s) \psi(s, x) d s+k_{2}\left(t_{k}\right) \psi^{\prime}\left(t_{k}, x\right) .
\end{aligned}
$$

Using these definitions, we can represent system (99) in the abstract form (1). Moreover, it is easy to see that $G, I_{k}$, and $J_{k}$ are continuous, and $G, I_{k}, J_{k}$, and $F$ are bounded linear operators with $E\|G\|_{L(\mathscr{B}, H)}^{p} \leq L_{G}, E\left\|I_{k}\right\|_{L(\mathscr{B}, H)}^{p} \leq c_{k}$, $E\left\|J_{k}\right\|_{L(\mathscr{B}, H)}^{p} \leq d_{k}, k=1,2, \ldots, m$, and $E\|F\|_{L(\mathscr{B}, H)}^{p} \leq L_{F}$, where $L_{G}=\left[\widetilde{L}_{1}+\left\|b_{2}\right\|_{\infty}\right]^{p}, c_{k}=\left[\vartheta_{k}+\left\|k_{1}\right\|_{\infty}\right]^{p}, d_{k}=$ $\left[\widetilde{\vartheta}_{k}+\left\|k_{2}\right\|_{\infty}\right]^{p}$, and $L_{F}=\left[\left\|a_{1}\right\|_{\infty} \widehat{L}_{1}+\left\|\varsigma_{1}\right\|_{\infty}+\left\|\varsigma_{2}\right\|_{\infty}\left\|a_{3}\right\|_{L^{1}} \widehat{L}_{2}+\right.$
$\left.\left\|\varsigma_{3}\right\|_{L^{1}}\right]^{p}$. Further, we can impose some suitable conditions on the above-defined functions to verify the assumptions on Theorem 16. Therefore, assumptions (H1)-(H6), (B1), and (B2) all hold, and the associated linear system of (99) is not exactly controllable but it is approximately controllable. Hence by Theorems 16 and 17, system (99) is approximately controllable on $[0, b]$.

\section{Conflict of Interests}

The author declares that there is no conflict of interests regarding the publication of this paper.

\section{Acknowledgments}

The author thanks the referees for their valuable comments and suggestions which improved their paper. This work is supported by the National Natural Science Foundation of China (Grant no. 11461019) and is supported by the President Found of Scientific Research Innovation and Application of Hexi University (Grant no. xz2013-10).

\section{References}

[1] M. Benchohra, J. Henderson, and S. K. Ntouyas, Impulsive Differential Equations and Inclusions, vol. 2 of Contemporary Mathematics and Its Applications, Hindawi Publishing Corporation, New York, New York, NY, USA, 2006.

[2] V. Lakshmikanthan, D. D. Bainov, and P. S. Simeonov, Theory of Impulsive Differential Equations, World Scientific Publishers, Singapore, 1989.

[3] W. M. Haddad, V. Chellaboina, and S. G. Nersesov, Impulsive and Hybrid Dynamical Systems: Stability, Dissipativity, and Control, Princeton Series in Applied Mathematics, Princeton University Press, Princeton, NJ, USA, 2006.

[4] E. Hernández, M. Pierri, and G. Goncalves, "Existence results for an impulsive abstract partial differential equation with statedependent delay," Computers \& Mathematics with Applications, vol. 52, no. 3-4, pp. 411-420, 2006.

[5] A. Anguraj and A. Vinodkumar, "Existence, uniqueness and stability results of impulsive stochastic semilinear neutral functional differential equations with infinite delays," Electronic Journal of Qualitative Theory of Differential Equations, vol. 67, pp. 1-13, 2009.

[6] A. Lin, Y. Ren, and N. Xia, "On neutral impulsive stochastic integro-differential equations with infinite delays via fractional operators," Mathematical and Computer Modelling, vol. 51, no. 5-6, pp. 413-424, 2010.

[7] Z. Yan and X. Yan, "Existence of solutions for impulsive partial stochastic neutral integrodifferential equations with statedependent delay," Collectanea Mathematica, vol. 64, no. 2, pp. 235-250, 2013.

[8] R. Triggiani, "A note on the lack of exact controllability for mild solutions in Banach spaces," SIAM Journal on Control and Optimization, vol. 15, no. 3, pp. 407-411, 1977.

[9] N. I. Mahmudov and A. Denker, "On controllability of linear stochastic systems," International Journal of Control, vol. 73, no. 2, pp. 144-151, 2000.

[10] J. P. Dauer and N. I. Mahmudov, "Controllability of stochastic semilinear functional differential equations in Hilbert spaces," 
Journal of Mathematical Analysis and Applications, vol. 290, no. 2, pp. 373-394, 2004.

[11] N. I. Mahmudov, "Controllability of linear stochastic systems," IEEE Transactions on Automatic Control, vol. 46, no. 5, pp. 724731, 2001.

[12] P. Balasubramaniam, J. Y. Park, and P. Muthukumar, "Approximate controllability of neutral stochastic functional differential systems with infinite delay," Stochastic Analysis and Applications, vol. 28, no. 2, pp. 389-400, 2010.

[13] R. Sakthivel, J. J. Nieto, and N. I. Mahmudov, "Approximate controllability of nonlinear deterministic and stochastic systems with unbounded delay," Taiwanese Journal of Mathematics, vol. 14, no. 5, pp. 1777-1797, 2010.

[14] R. Subalakshmi and K. Balachandran, "Approximate controllability of nonlinear stochastic impulsive integrodifferential systems in Hilbert spaces," Chaos, Solitons \& Fractals, vol. 42, no. 4, pp. 2035-2046, 2009.

[15] L. Shen and J. Sun, "Approximate controllability of stochastic impulsive functional systems with infinite delay," Automatica, vol. 48, no. 10, pp. 2705-2709, 2012.

[16] Y. Zang and J. Li, "Approximate controllability of fractional impulsive neutral stochastic differential equations with nonlocal conditions," Boundary Value Problems, vol. 2013, article 193, 14 pages, 2013.

[17] Y. Ren and D. Sun, "Second-order neutral impulsive stochastic evolution equations with delay," Journal of Mathematical Physics, vol. 50, Article ID 102709, 2009.

[18] J. Cui and L. Yan, "Existence results for impulsive neutral second-order stochastic evolution equations with nonlocal conditions," Mathematical and Computer Modelling, vol. 57, no. 9-10, pp. 2378-2387, 2013.

[19] N. I. Mahmudov and M. A. McKibben, "Approximate controllability of second-order neutral stochastic evolution equations," Dynamics of Continuous, Discrete and Impulsive Systems B, vol. 13, pp. 619-634, 2006.

[20] P. Muthukumar and P. Balasubramaniam, "Approximate controllability of second-order damped McKean-Vlasov stochastic evolution equations," Computers \& Mathematics with Applications, vol. 60, no. 10, pp. 2788-2796, 2010.

[21] P. Balasubramaniam and P. Muthukumar, "Approximate controllability of second-order stochastic distributed implicit functional differential systems with infinite delay," Journal of Optimization Theory and Applications, vol. 143, no. 2, pp. 225-244, 2009.

[22] R. Sakthivel, Y. Ren, and N. I. Mahmudov, "Approximate controllability of second-order stochastic differential equations with impulsive effects," Modern Physics Letters B: Condensed Matter Physics, Statistical Physics, Applied Physics, vol. 24, no. 14, pp. 1559-1572, 2010.

[23] R. Pettersson, "Existence theorem and Wong-Zakai approximations for multivalued stochastic differential equations," Probability and Mathematical Statistics, vol. 17, pp. 29-45, 1997.

[24] N. U. Ahmed, "Nonlinear stochastic differential inclusions on Banach space," Stochastic Analysis and Applications, vol. 12, no. 1, pp. 1-10, 1994.

[25] P. Balasubramaniam and S. K. Ntouyas, "Controllability for neutral stochastic functional differential inclusions with infinite delay in abstract space," Journal of Mathematical Analysis and Applications, vol. 324, no. 1, pp. 161-176, 2006.

[26] Z. Yan and X. Yan, "Existence of solutions for a impulsive nonlocal stochastic functional integrodifferential inclusion in
Hilbert spaces," Zeitschrift für Angewandte Mathematik und Physik, vol. 64, no. 3, pp. 573-590, 2013.

[27] Z. Yan and H. Zhang, "Existence of impulsive fractional partial neutral stochastic integro-differential inclusions with statedependent delay in Hilbert spaces," Electronic Journal of Differential Equations, vol. 2013, pp. 1-21, 2013.

[28] B. C. Dhage, "Fixed-point theorems for discontinuous multivalued operators on ordered spaces with applications," Computers \& Mathematics with Applications, vol. 51, no. 3-4, pp. 589-604, 2006.

[29] K. Deimling, Multi-Valued Differential Equations, De Gruyter, Berlin, Germany, 1992.

[30] S. Hu and N. Papageorgiou, Handbook of Multivalued Analysis, Kluwer Academic Publishers, Dordrecht, The Netherlands, 1997.

[31] J. Kisyński, "On cosine operator functions and one parameter group of operators," Studia Mathematica, vol. 49, pp. 93-105, 1972.

[32] C. C. Travis and G. F. Webb, "Cosine families and abstract nonlinear second order differential equations," Acta Mathematica Academiae Scientiarum Hungaricae, vol. 32, no. 1-2, pp. 75-96, 1978.

[33] C. C. Travis and G. F. Webb, "Compactness, regularity, and uniform continuity properties of strongly continuous cosine families," Houston Journal of Mathematics, vol. 3, no. 4, pp. 555$567,1977$.

[34] J. K. Hale and J. Kato, "Phase space for retarded equations with infinite delay," Funkcialaj Ekvacioj. Serio Internacia, vol. 21, no. 1, pp. 11-41, 1978.

[35] Y. Hino, S. Murakami, and T. Naito, Functional-Differential Equations with Infinite Delay, vol. 1473 of Lecture Notes in Mathematics, Springer, Berlin, Germany, 1991.

[36] H. R. Henríquez and C. H. Vásquez, "Differentiability of solutions of second-order functional differential equations with unbounded delay," Journal of Mathematical Analysis and Applications, vol. 280, no. 2, pp. 284-312, 2003.

[37] G. Da Prato and J. Zabczyk, Stochastic Equations in Infinite Dimensions, Cambridge University Press, Cambridge, UK, 1992.

[38] A. Lasota and Z. Opial, "An application of the Kakutani-KyFan theorem in the theory of ordinary differential equations," Bulletin de l'Académie Polonaise des Sciences, Série des Sciences Mathématiques, Astronomiques et Physiques, vol. 13, pp. 781-786, 1965. 


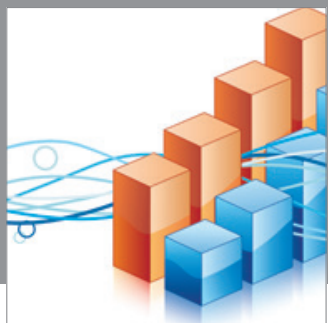

Advances in

Operations Research

mansans

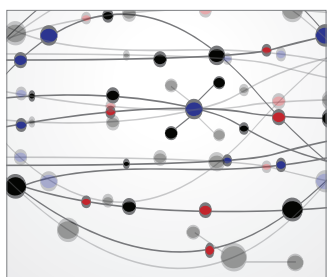

The Scientific World Journal
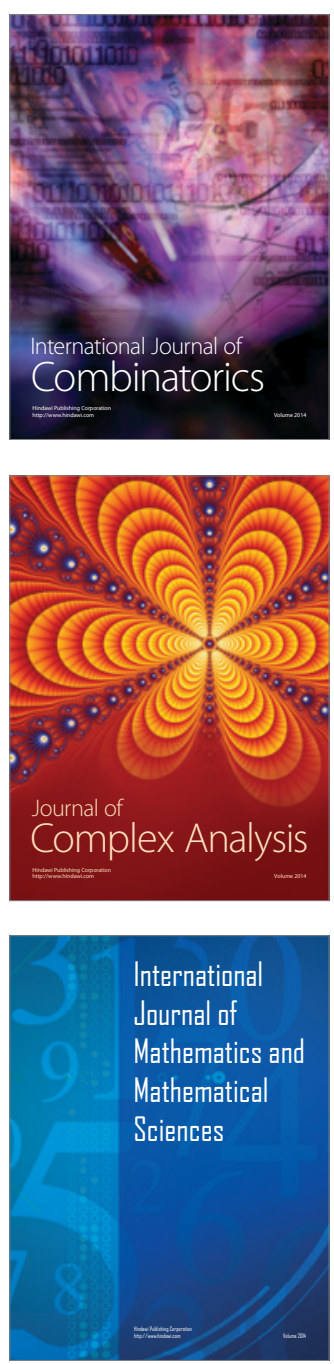
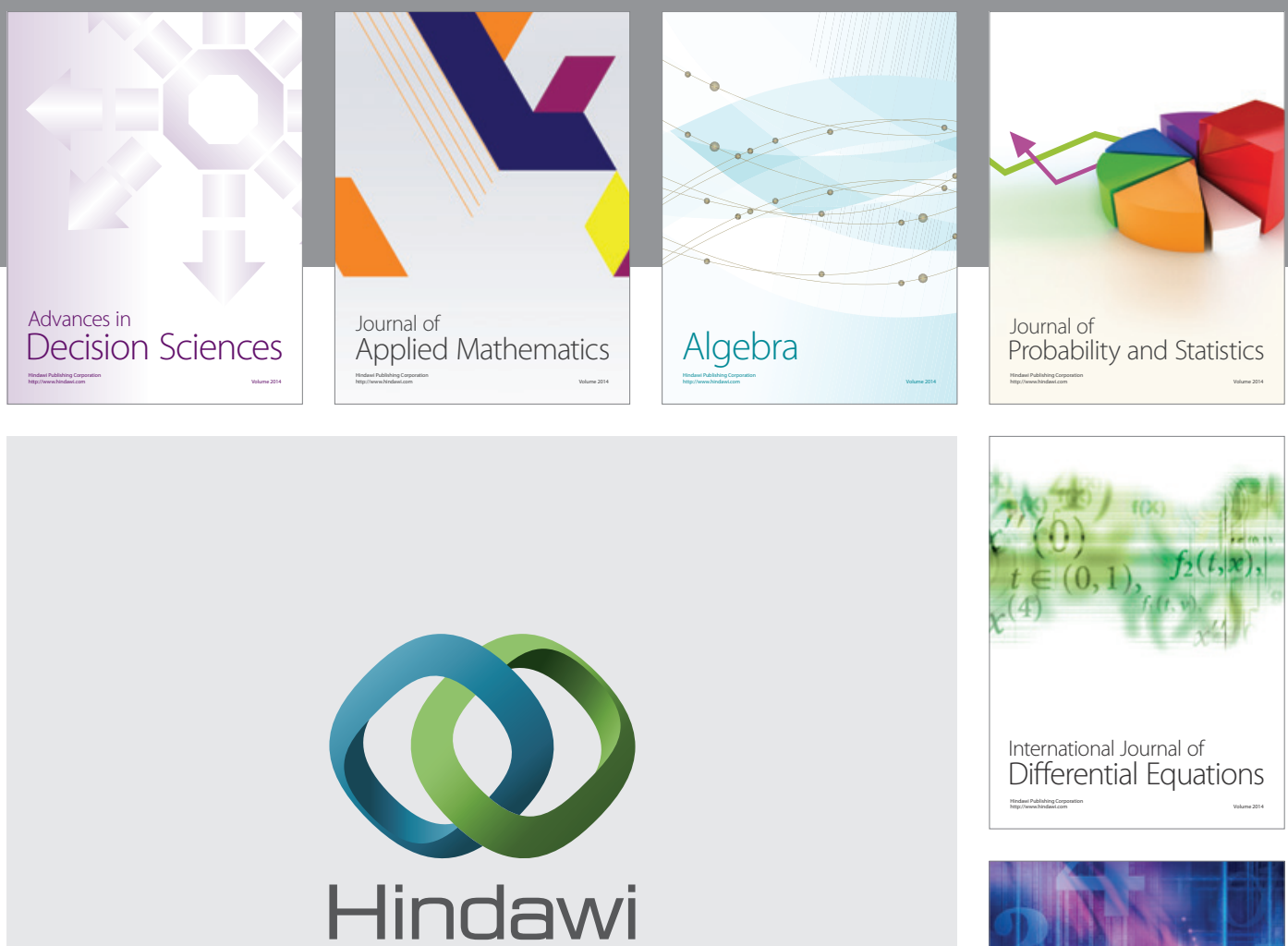

Submit your manuscripts at http://www.hindawi.com
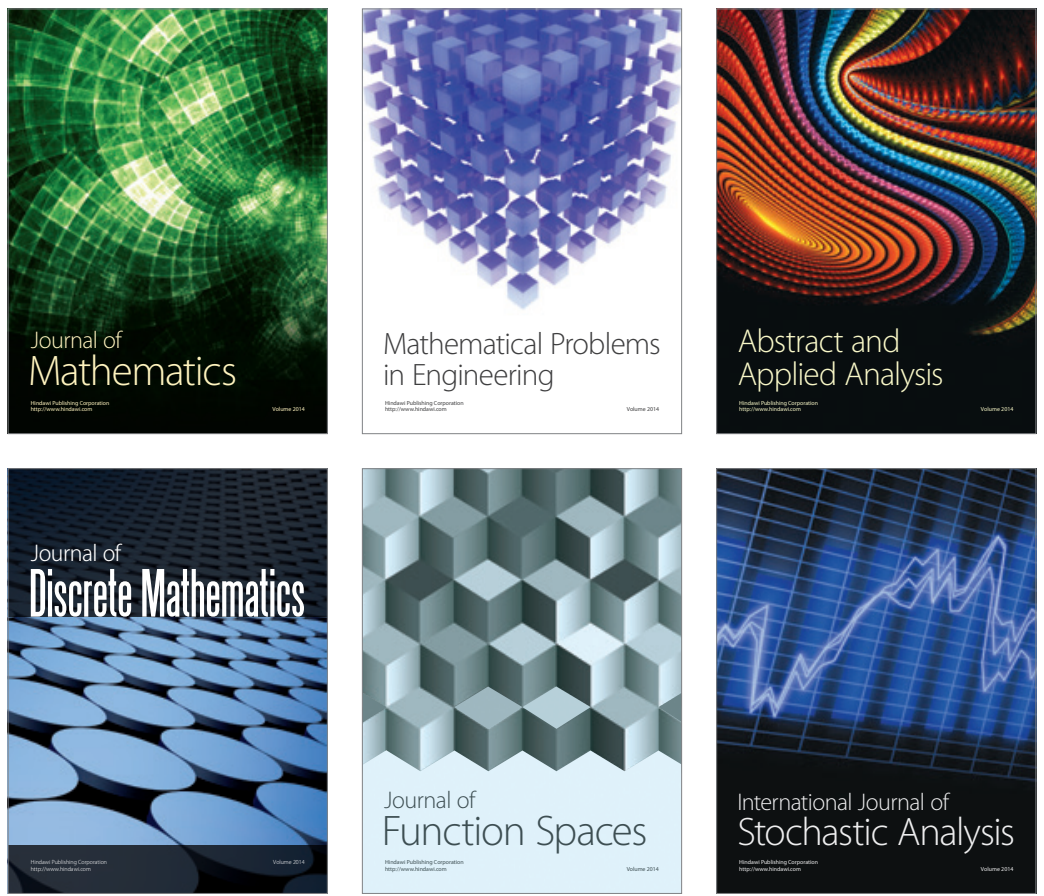

Journal of

Function Spaces

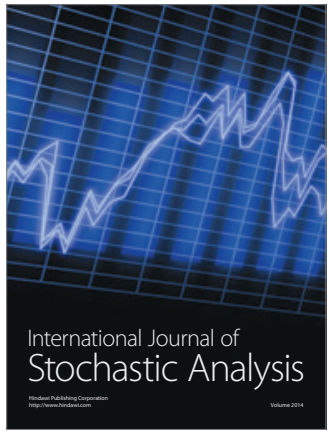

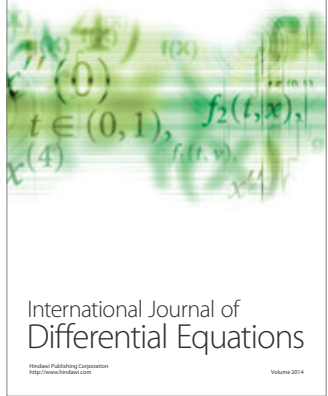
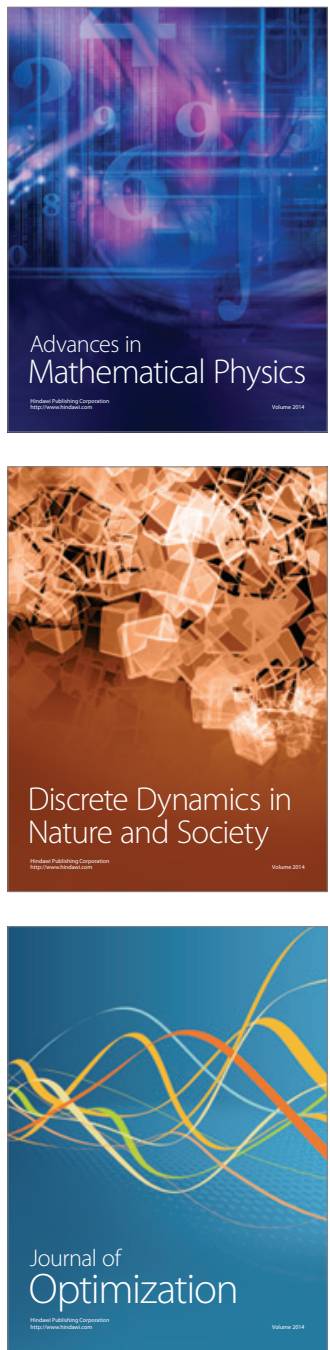